\title{
How was Dark Matter produced in the early universe? A study of fermionic Dark Matter with a Z-prime portal
}

\author{
by \\ Taylor Rose Gray \\ A thesis submitted to the \\ Faculty of Graduate and Postdoctoral Affairs \\ in partial fulfillment of the requirements \\ for the degree of \\ Master of Science \\ in \\ Particle Physics \\ Department of Physics \\ Carleton University \\ Ottawa-Carleton Institute of Physics \\ Ottawa, Canada
}

Copyright (c) 2020

Taylor Rose Gray 


\section{Abstract}

We study a model of fermionic dark matter interacting with the Standard Model through a sequential $Z^{\prime}$ mediator, the gauge boson of a $U(1)$ extension to the Standard Model symmetry group, to understand the mechanism responsible for the dark matter relic abundance. We compare two different mechanisms for dark matter production in the early universe, freeze-out and freeze-in. For production through freeze-out, dark matter particles were in thermal equilibrium with the cosmic plasma until the expansion of the universe dominated over the interactions. For freeze-in, dark matter was never in thermal equilibrium with the visible sector, and its abundance is always negligible compared to the thermal bath abundances. The boundary between each production regime is explored by considering every free parameter of this model, namely the masses and couplings. Details of the boundary region between freeze-in and freeze-out could have implications for dark matter searches. 


\section{Acknowledgments}

Throughout the entire process that writing this thesis entailed, I have received an immense amount of support, guidance, and patience. First, I wish to show my deepest gratitude to my supervisor, Stephen Godfrey, who has taught me more than I could have imagined learning in my degree. Not only has Steve gone above and beyond helping me with my thesis research and writing, but he also has made the experience very enjoyable by being so pleasant to work with. I would like to thank Catarina Cosme, who has played an essential role in our research. I am indebted to all of the knowledge Catarina has shared with me, and all of the help she has been with this thesis. I would also like to thank Maíra Dutra for all that she has contributed to my success. I am so grateful of Maíra's patience while helping me with detailed calculations and while answering all of my questions. I greatly appreciate all of the time and effort that Maíra, Catarina, and Steve have sacrificed for this work. I'd like to recognize the Theory Group at Carleton for all of the seminars and journal clubs which I have been lucky to be part of and learn from. I am also very grateful to all of the wonderful professors I have had for my Master's level courses. I have made lasting friendships among my peers that have helped make my Master's experience a delight. I thank them all for their support and of course their company at the pub or while playing hours of board games at Jérôme's. I also have my skateboard friends to thank, who are all the most positive, fun, and encouraging people to spend 
your free time with. I am forever thankful for my entire family, Dad, Mom, Jesse, Gran, and Gramps (and not to forget Zeppelin and RockC) for their love and support throughout my studies. Finally, I cannot begin to express how grateful I am for all that Nick does for me. He inspires me everyday to be my best self and is constantly there for me. His encouragement and love have made my Master's experience a lot easier and so enjoyable. I can't wait to see what the future has in store for us. 


\section{Contents}

$\begin{array}{ll}\text { Abstract } & \text { ii }\end{array}$

Acknowledgments

List of Tables $\quad$ viii

List of Figures $\quad$ xiv

1 Introduction 1

1.1 Evidence for Dark Matter . . . . . . . . . . . . . . . . 3

1.2 How do we solve the Dark Matter problem? . . . . . . . . . 7

2 Dark Matter Production Mechanisms 10

2.1 Freeze-Out Mechanism . . . . . . . . . . . . . . . . . . . . . 10

2.2 Freeze-In Mechanism . . . . . . . . . . . . . . . . . . . . . . . . . . 12

2.3 Other Mechanisms . . . . . . . . . . . . . . . . . . . . 14

2.3.1 Dark Freeze-Out . . . . . . . . . . . . . . . 14

2.3 .2 Reannihilation. . . . . . . . . . . . . . . . 15

2.3 .3 Non-Thermal Candidates . . . . . . . . . . . . . . . . 16

3 Computing the Dark Matter Abundance: The Boltzmann Equation 19 
3.1 Equilibrium Thermodynamics . . . . . . . . . . . . . . . 20

3.2 The Boltzmann Equation . . . . . . . . . . . . . . . . . 23

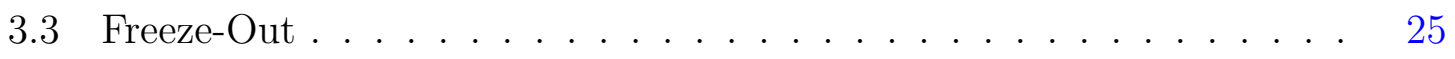

3.3.1 Boltzmann Equation for $12 \leftrightarrow 34 \ldots \ldots \ldots \ldots$

3.3.2 Boltzmann Equation for $1 \leftrightarrow 34 \ldots \ldots \ldots \ldots 27$

3.3.3 Freeze-Out Approximation . . . . . . . . . . . . . . . . . . 29

3.4 Freeze-In Approximation . . . . . . . . . . . . . . . . . . . . . . . 32

3.5 Interaction Rates $\ldots \ldots \ldots \ldots \ldots$

4 Model 36

4.1 Lagrangian . . . . . . . . . . . . . . . . 36

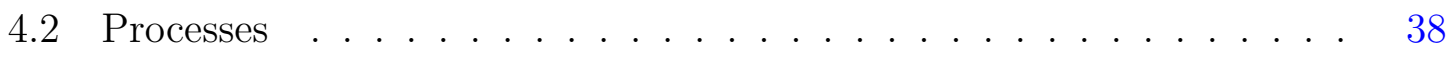

$4.2 .1 \quad Z^{\prime} Z^{\prime} \leftrightarrow \chi \bar{\chi} \quad \ldots \ldots \ldots \ldots \ldots \ldots \ldots \ldots \ldots$

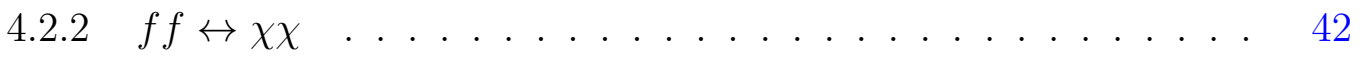

4.3 UV Completion . . . . . . . . . . . . . . . . . 46

5 Results 48

5.1 Determining the Production Regime $\ldots \ldots \ldots \ldots$

5.2 Dark Matter Evolution . . . . . . . . . . . . . . . . . 51

5.3 Relic Abundance . . . . . . . . . . . . . . . . . . . . . 56

6 Conclusion $\quad 65$

6.1 Future Directions . . . . . . . . . . . . . . . . . . 65

6.2 Concluding Remarks . . . . . . . . . . . . . . 67

$\begin{array}{lll}\text { Appendix A Amplitudes and Cross Sections } & 70\end{array}$

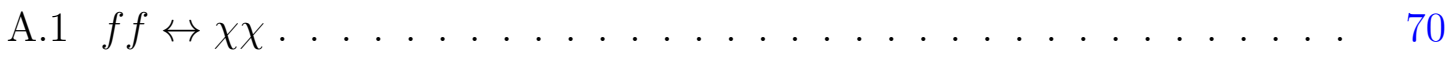




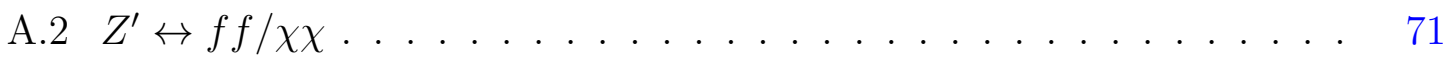

A.3 $Z^{\prime} Z^{\prime} \leftrightarrow \chi \chi \ldots \ldots \ldots \ldots \ldots \ldots \ldots 72$

References 


\section{List of Tables}

4.1 Values of $V_{f}$ and $A_{f}$, the axial and vector $Z^{\prime}$ Standard Model fermion

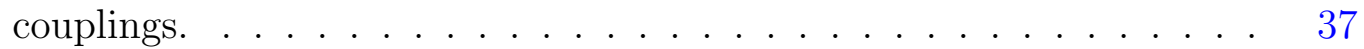




\section{List of Figures}

1.1 The particles that make up the Standard Model. . . . . . . . . . . . . 1

1.2 Image of the Bullet Cluster (1E 0657-558) showing baryonic matter in red measured by the Chandra telescope, and dark matter in blue measured by gravitational lensing [12]. The baryonic matter is separate from the dark matter, indicating that dark matter is some other form of matter that is non-baryonic. . . . . . . . . . . . . .

1.3 Galaxy rotation curve of NGC 6503. Circular velocities of gas or stars in the galaxy as a function of radial distance from the galactic center [14]. 5

1.4 A sky map showing temperature anisotropies of the Cosmic Microwave Background (CMB) on the order of $10^{-5}$ observed by Planck [21]. . . 6

1.5 The temperature power spectrum of the CMB measured by Planck [20]. The fit of the theoretical $\Lambda$ CDM spectrum is plotted in light blue. .

2.1 The comoving number density as a function of $x \equiv \frac{m}{T}$ where $m$ is the dark matter mass for the freeze-out scenario, plotted for a generic constant cross section at different couplings $(\lambda)$, represented by coloured lines. The dashed black line is the equilibrium yield assuming MaxwellBoltzmann statistics. . . . . . . . . . . . . . . . 11 
2.2 The comoving number density as a function of $x \equiv \frac{m}{T}$ where $m$ is the dark matter mass for the freeze-in scenario. Plotted for a generic constant cross section at different couplings $(\lambda)$, represented by coloured lines. The dashed black line is the equilibrium yield assuming MaxwellBoltzmann statistics. Notice that the yield of dark matter is much less than the equilibrium yield before freeze-in occurs. . . . . . . . . . . . 13

2.3 Two scenarios of the evolution of two dark sector species. Species 1 thermalizes within the dark sector (which is represented in blue) and species 2 does not thermalize and freezes-in (which is represented in red). The yield $Y \equiv \frac{n}{s}$ is plotted as a function of $x \equiv \frac{m_{1}}{T}$. The dashed blue curves are the equilibrium curves of species 1 . This figure comes from Ref. [63]. . . . . . . . . . . . . . . . . .

2.4 The evolution of dark matter in the case of reannihilation (in black), freeze-in (in dashed blue), and freeze-out (in dashed red). The yield $Y \equiv \frac{n}{s}$ is plotted as a function of $x \equiv \frac{m}{T}$. The equilibrium yield is plotted in dashed grey. This figure is from Ref. [53]. . . . . . . . . . .

4.1 The Feynman diagrams for the dark matter production processes in our model. Subfigure (a) is the $Z^{\prime}$ decay into dark matter, subfigure (b) is the s-channel Standard Model fermion annihilation into dark matter through the $Z^{\prime}$, finally (c) and (d) are the t and $\mathrm{u}$-channels respectively for the $Z^{\prime}$ annihilation into dark matter through the dark matter fermion. . . . . . . . . . . . . . .

4.2 Feynman diagram for the $\chi \chi \leftrightarrow \chi \chi \chi \chi$ process which could lead to a dark sector freeze-out (not considered in this work). . . . . . . . . 
4.3 Feynman diagrams for scattering processes which may lead to kinetic equilibrium between $\chi, Z^{\prime}$, and the Standard Model sector. . . . . .

4.4 The production rate density for $Z^{\prime}$ annihilation (t/u-channel) is plotted as a function of temperature. The full squared amplitude numerically integrated over $s_{m}$ and $\theta$ (solid black) and under the approximation $\sqrt{s_{m}} \sim T \gg m_{Z^{\prime}}$ (dashed blue) are compared. The line corresponding to $T=m_{Z^{\prime}}$ is plotted in red. At high temperatures $\left(T>m_{Z^{\prime}}\right) R \propto T^{6}$, and at low temperatures $\left(T<m_{Z^{\prime}}\right)$ the $Z^{\prime}$ abundance is Boltzmann suppressed thus $R$ drops near zero. . . . . . . . . . . . .

4.5 The production rate density for electron positron annihilation (s-channel) is plotted as a function of temperature. The full squared amplitude numerically integrated over $s_{m}$ (solid black) and under the three approximations of each temperature regime (dashed coloured) are compared. The lines corresponding to $T=m_{Z^{\prime}}$ and $T=m_{\chi}$ are plotted as red and purple vertical lines respectively. For high temperatures $R \propto T^{4}$ and for low temperatures, but for $T>m_{\chi}, R \propto T^{7} \ldots \ldots \ldots$

5.1 The ratio of the production frequency labeled $W$ to the Hubble parameter labeled $H$ as a function of $\mathrm{x}$ for various dark matter couplings. All three production channels are shown. The dashed black lines show where the ratio is equal to 1 (where $W=H$ ). If at least one of the processes is above the dashed line we assume that chemical equilibrium is reached and dark matter is produced through freeze-out (FO). If no processes have a large enough $W$ then we assume dark matter is produced through freeze-in (FI). A specific example is shown where $m_{\chi}=1000 \mathrm{GeV}$ and $m_{Z^{\prime}}=10^{6} \mathrm{GeV} \ldots \ldots \ldots$. . . . . . . . 50 
5.2 The evolution of the yield of dark matter for different couplings $\left(A_{\chi}, V_{\chi}\right)$. The horizontal axis is $x=\frac{m_{\chi}}{T}$ which flows in the direction of time. The red dashed line shows the yield which gives the observed relic abundance, $\Omega h^{2}=0.12$ [20]. The top left plot has $m_{Z^{\prime}}=10^{3} \mathrm{GeV}$ and $m_{\chi}=10 \mathrm{GeV}$. The top right has $m_{Z^{\prime}}=10^{3} \mathrm{GeV}$ and $m_{\chi}=10^{4} \mathrm{GeV}$. The bottom left has $m_{Z^{\prime}}=10^{8} \mathrm{GeV}$ and $m_{\chi}=10 \mathrm{GeV}$. The bottom right has $m_{Z^{\prime}}=10^{8} \mathrm{GeV}$ and $m_{\chi}=10^{4} \mathrm{GeV}$. . . . . . . . . . . 52

5.3 The interaction rate densities, $R$, for all three production processes at different $Z^{\prime}$ and $\chi$ masses as a function of $x=\frac{m_{\chi}}{T} . A_{\chi}=V_{\chi}=10^{-10}$, corresponding to the freeze-in regime, for all four subfigures. $R_{f f \rightarrow \chi \chi}$ is plotted in blue, $R_{Z^{\prime} Z^{\prime} \rightarrow \chi \chi}$ is in orange, and $R_{Z^{\prime} \rightarrow \chi \chi}$ in green. The vertical lines correspond to $T=m_{Z^{\prime}}$ (red) and $T=m_{\chi}$ (purple). . .

5.4 The interaction rate densities, $R$, for all three production processes at different $Z^{\prime}$ and $\chi$ masses as a function of $x=\frac{m_{\chi}}{T} . A_{\chi}=V_{\chi}=10^{-3}$ for all four subfigures, corresponding to the freeze-out regime. $R_{f f \rightarrow \chi \chi}$ is plotted in blue, $R_{Z^{\prime} Z^{\prime} \rightarrow \chi \chi}$ is in orange, and $R_{Z^{\prime} \rightarrow \chi \chi}$ in green. The vertical lines correspond to $T=m_{Z^{\prime}}$ (red) and $T=m_{\chi}$ (purple). . . 
5.5 Contour plot showing the relic abundance as a function of dark matter couplings and $m_{Z^{\prime}}$ for $m_{\chi}=1000 \mathrm{GeV}$. The dashed line is the freezein/out boundary which is determined by comparing the frequency of interactions $W$ to Hubble $H$ for each process and each temperature from reheating until Boltzmann suppression. All values below this boundary are classified as freeze-in and above as freeze-out. The observed relic density is plotted in red for both freeze-out and freeze-in regimes [20]. Notice that the relic abundance in the freeze-out regime decreases as couplings increase, and that the freeze-in relic abundance increases with couplings. . . . . . . . . . . . . .

5.6 Contour lines representing the observed relic abundance $\Omega h^{2}=0.12$ [20] as a function of dark matter couplings and $m_{Z^{\prime}}$ at four $m_{\chi}$ values. The dashed lines represent the boundary between production regimes, freeze-in and freeze-out. . . . . . . . . . . . . . .

5.7 Contour lines representing the observed relic abundance $\Omega h^{2}=0.12$ [20] as a function of $m_{\chi}$ and $m_{Z^{\prime}}$ for various dark matter couplings, $A_{\chi}$ and $V_{\chi}$ where $A_{\chi}=V_{\chi}$, each represented by a different colour. The observed abundance in the freeze-in regime is represented as dashed lines, while the observed abundance in the freeze-out regime is represented by solid lines. The regime boundaries are plotted in dotted lines. The only coupling which has a regime boundary in this parameter space is $10^{-8}$, shown as purple dotted lines while each regime is labeled by purple text. The rest of the couplings are either entirely in the freeze-in or freeze-out regime. . . . . . . . . . . . . . . . . 
5.8 The contour lines of the correct relic abundance $\Omega h^{2}=0.12[20]$ as a function of $m_{\chi}$ and $m_{Z^{\prime}}$ for the case of $V_{\chi}=0$ [104]. Each colour represented a different Axial dark matter coupling. $m_{\chi}=m_{Z^{\prime}}$ is shown in black, and $2 m_{\chi}=m_{Z^{\prime}}$ is plotted in green. Couplings are large enough such that the entire parameter space is in the freeze-out regime. 63 


\section{Chapter 1}

\section{Introduction}

Our current understanding of the basic building blocks of the universe and the forces that bind them together is far from complete. The Standard Model of particle physics has been an extremely successful theory that has made many predictions which have been verified. The Standard Model describes the subatomic particles and all of the forces that allow them to interact (except for gravity) as illustrated in Figure 1.1. For instance, the Higgs boson is predicted by the Standard Model and was discovered in 2012 by the ATLAS and CMS collaborations $[1,2]$. The existence of other particles

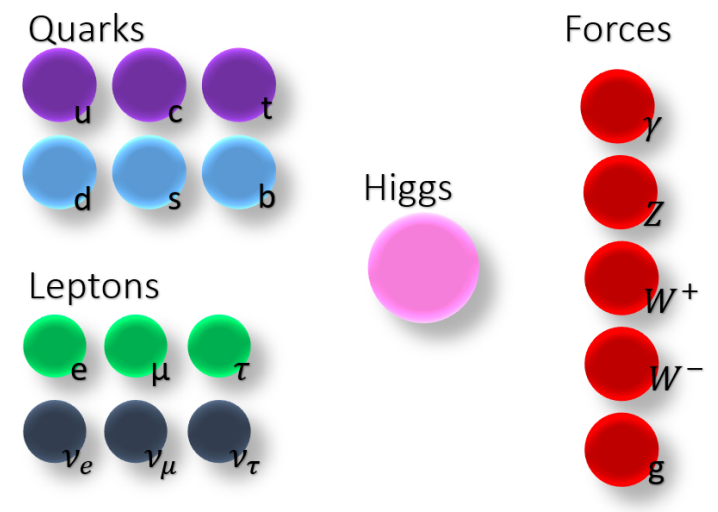

Figure 1.1: The particles that make up the Standard Model. 
such as the top quark [3] and $\mathrm{W}$ and $\mathrm{Z}$ bosons [4] have also been predicted by the Standard Model and later discovered. Despite the Standard Model's successes, it fails to give a complete explanation of a number of observations and phenomena such as matter anti-matter asymmetry, gravity, dark energy ${ }^{1}$, the hierarchy problem ${ }^{2}$, neutrino masses, and dark matter [5]. This thesis focuses on addressing the latter problem. Dark matter is widely accepted to be a particle or an entire sector of particles of unknown nature beyond the Standard Model yet to be discovered. Its evidence is overwhelming on all scales in the universe and major efforts are in place to discover it.

This thesis begins with a historical overview of how we came to know of the existence of dark matter, and then a review of what we know about dark matter and the efforts to solve this problem. In Chapter 2 we will then describe different ways that dark matter could be produced in the early universe, focusing on freeze-in and freezeout mechanisms. Next, Chapter 3 goes over necessary concepts in thermodynamics and the methods to calculate the dark matter relic abundance as a function of time using the Boltzmann equation. We will describe our dark matter model in Chapter 4 and the processes that arise from it. The way in which we differentiate between the dark matter production regimes is explained in Chapter 5 along with the main results, namely the evolution of dark matter in our model for each production regime, the relic abundance as a function of the free parameters of our model, the production regime boundary, and which parameter values are consistent with the observed abundance. We summarize our findings and discuss future directions in Chapter 6 .

\footnotetext{
${ }^{1}$ Dark energy is the unknown force which causes the universe's accelerated expansion.

${ }^{2}$ The hierarchy problem refers to the large difference between the Higgs and gauge boson masses compared to the Planck mass.
} 


\subsection{Evidence for Dark Matter}

Throughout astronomical history, there were discussions about the nature of "dark" objects such as unobserved planets and dark patches in the sky [6]. In 1844 Friederich Bessel wrote about how there must be unobserved stars influencing the motion of the stars Sirius and Procyon [7]. This was one of the first times in history that an astronomical object was predicted solely from its gravitational interactions [8]. Lord Kelvin estimated the amount of matter in the Milky Way in 1904 from measurements of velocities of stars and concluded that it is likely that many stars are dark bodies which we cannot see [9]. Fritz Zwicky was one of the first astronomers to predict the existence of dark matter. In 1933 Zwicky studied the velocities of galaxies in the Coma Cluster from measurements of redshifts and compared them to a rough estimate of the velocities using the virial theorem [10], finding a large discrepancy between the measurements and his predictions. He noticed that the velocities of 800 galaxies each $10^{9}$ solar masses within a radius of one million light years should be approximately $80 \mathrm{~km} / \mathrm{s}$, although measurements showed that they were approximately $1000 \mathrm{~km} / \mathrm{s}$. The fact that the measured velocities are far greater than predicted is evidence that there is unobserved matter in galaxy clusters that can account for this discrepancy, which Zwicky dubbed dark matter.

There is evidence for dark matter on all scales in the universe. On the scale of galaxy clusters, the Bullet Cluster (1E 0657-56) [11] shown in Figure 1.2, elegantly demonstrates the existence of dark matter. The Bullet Cluster is the result of a collision of two galaxy clusters, where each cluster has a baryonic component and a dark matter component. The pink/red clumps in Figure 1.2 show the X-Rays emitted from hot baryonic gas detected by the Chandra telescope. The blue clumps show where the 


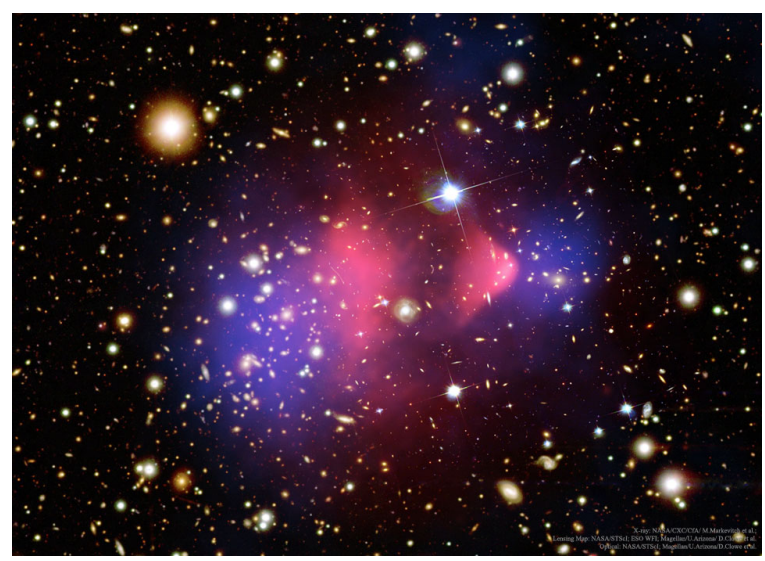

Figure 1.2: Image of the Bullet Cluster (1E 0657-558) showing baryonic matter in red measured by the Chandra telescope, and dark matter in blue measured by gravitational lensing [12]. The baryonic matter is separate from the dark matter, indicating that dark matter is some other form of matter that is non-baryonic.

mass is observed to be concentrated from gravitational lensing measurements by the Hubble Space Telescope and the Magellan Optical Telescopes. Gravitational lensing, a method that uses the fact that light from distant objects is bent and distorted by gravitational fields, is used to map the distribution of mass in the cluster [13]. As evident by Figure 1.2, the observed mass does not overlap with the majority of the mass, implying that the majority of the matter is non-baryonic and does not emit any radiation, therefore it is "dark". The observed matter in both clusters is located towards the center of the collision while each dark matter component is further from the center, supporting the idea that dark matter is very weakly interacting compared to ordinary matter which feels slowing forces of friction.

On the galactic scale there is evidence for dark matter in rotation curves of stars in galaxies [14]. It is expected that the circular velocities $v(r)$ of stars in a galaxy are described by,

$$
v(r)=\sqrt{\frac{G M(r)}{r}}
$$

past the optical disk, where $r$ is the distance from the galactic center, $G$ is the 


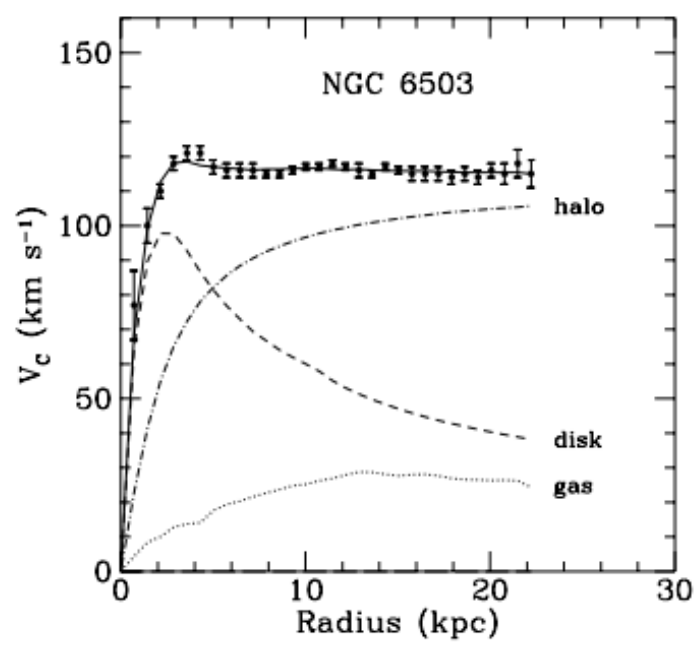

Figure 1.3: Galaxy rotation curve of NGC 6503. Circular velocities of gas or stars in the galaxy as a function of radial distance from the galactic center [14].

gravitational constant, and

$$
M(r)=4 \pi \int_{0}^{r} \rho\left(r^{\prime}\right) r^{\prime 2} d r^{\prime}
$$

where $\rho$ is the density distribution [15]. Instead, measurements show that the circular velocity curves flat-line as in Figure 1.3. This flattening of $v(r)$ indicates that $M(r) \propto r$ and $\rho(r) \propto \frac{1}{r^{2}}$, showing evidence for massive dark matter halos surrounding the galaxies [16]. The authors of Reference [14] concluded that this discrepancy between expected and measured circular velocities could be accounted for by Modified Newtonian Dynamics (MOND) [17]. Although MOND could potentially account for the rotational velocity curves, it simply cannot explain observations at larger scales such as large-scale structure, cosmic microwave background (CMB) anisotropies [18], and it certainly cannot explain the observations of the Bullet Cluster [13].

Dark matter played a fundamental role in the formation of large scale structure since it makes up most of the mass in the universe [19]. There is evidence for dark 


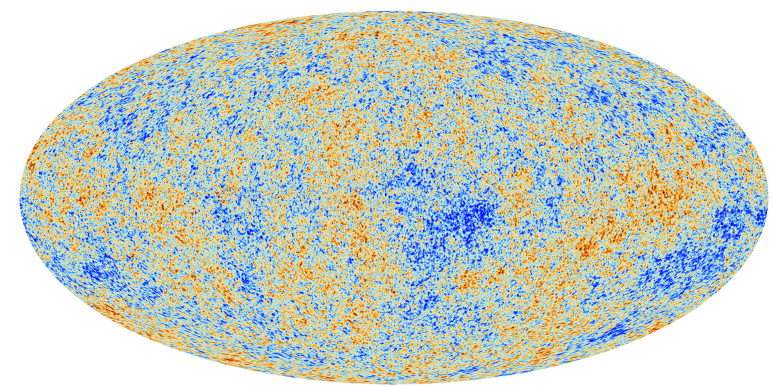

Figure 1.4: A sky map showing temperature anisotropies of the Cosmic Microwave Background (CMB) on the order of $10^{-5}$ observed by Planck [21].

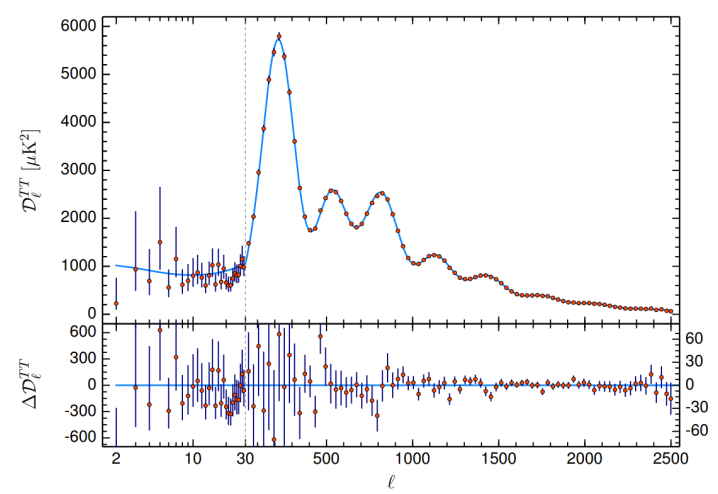

Figure 1.5: The temperature power spectrum of the CMB measured by Planck [20]. The fit of the theoretical $\Lambda \mathrm{CDM}$ spectrum is plotted in light blue.

matter in the CMB, shown in Figure 1.4, photons from when the universe first became transparent [20]. The angular fluctuations of the CMB temperature are caused by oscillations of the photon and baryon plasma in the early universe before the photons decoupled. The peaks and troughs in the power spectrum of these temperature anisotropies depict the angular scales of the CMB, and shows the individual effects of dark matter and baryonic matter, see Figure 1.5 [22]. Fitting the accepted model of modern cosmology, which is referred to as the $\Lambda$ CDM (cold dark matter) model [23], to this power spectrum gives the dark energy density, baryon density, and dark matter density. It turns out the universe is currently $69 \%$ dark energy, $26 \%$ dark matter, and $5 \%$ ordinary/baryonic matter [20]. Clearly we have a very large problem on our 
hands: what is the nature of the majority of the matter in the universe and when was it produced?

\subsection{How do we solve the Dark Matter problem?}

Ever since this concept of dark matter fell into our hands, efforts have been made to explain these gravitational observations. Neutrinos were proposed as a dark matter candidate since they interact only through weak and gravitational forces. These particles were hot (relativistic) during structure formation [24] and since simulations showed that for structure to form as we observe today dark matter must be cold (non-relativistic), Standard Model neutrinos were ruled out [25] as a candidate for dark matter. Another reason why Standard Model neutrinos could not account for all of the dark matter in the universe is that the density of fermions cannot exceed that of a degenerate Fermi gas, so there exists a lower bound on the mass called the "Tremaine-Gunn bound" [26]. Neutrinos are the only stable and electrically neutral particle in the Standard Model and cannot account for all of the dark matter in our universe, therefore we may conclude that if dark matter is a particle, it must be beyond the Standard Model. A natural solution is to postulate the existence of heavy sterile neutrinos. These particles are similar to Standard Model neutrinos but are much heavier and interact very weakly. Heavy sterile neutrinos are a viable dark matter candidate since they are cold during structure formation and are able to overcome constraints from structure formation [27]. Nevertheless, there are other dark matter candidates that have been proposed. For instance, primordial black holes were proposed to make up dark matter, although gravitational lensing experiments have set stringent constraints $[28,29]$. It is possible that black holes could account for all of the dark matter but only for certain mass windows [30]. There exists some theories 
that assume dark matter does not even exist and that the gravitational observations are due to our lack of understanding of gravity. As mentioned earlier, MOND is a way of getting around the idea that dark matter is an undiscovered kind of matter but rather an incompleteness of our Newtonian theory of gravity [31-33]. However, MOND can only account for smaller scale observations such as rotation curves of galaxies and fails at the scale of galaxy clusters and beyond [34]. MOND is in tension with observations of the Bullet Cluster since it cannot explain the separation of visible and invisible matter [13], and fails to provide an explanation for the oscillations in the CMB. In addition, experiments were conducted to test for departures from Newton's second law and none were found $[35,36]$.

For the reasons explained above, to our knowledge, dark matter is most likely an undiscovered particle. There are a large number of proposed dark matter particle candidates that are an attempt to explain the observed abundance [37]. In particular, some candidates attempt to explain problems in particle physics in addition to dark matter [38]. In 1977, Roberto Peccei and Helen Quinn proposed an additional $U(1)$ gauge symmetry in order to address the strong-CP problem, an issue with Quantum Chromodynamics (QCD) [39,40], which brings about a new particle called the axion $[41,42]$. However, the most popular dark matter candidates are the Weakly Interacting Massive Particles (WIMPs) which are produced through the freeze-out mechanism. More detail is given about this class of dark matter candidates in Chapter 2, but we wish to point out that since it can explain the observed abundance while the cross sections and masses are on the order of the weak scale [43-46], they are an attractive dark matter candidate because new physics due to the hierarchy problem [47] is expected to arise near the weak scale and they can be probed in current experiments. In fact, there are several experiments that are attempting to search for WIMPs [48], 
such as DEAP-3600 [49], LUX [50], XENON1T [51], and SuperCDMS [52] which attests the efforts that are being made to detect them. Unfortunately, there has yet to be any evidence of dark matter from these experiments and the parameter space of WIMPs is shrinking as experiments become more sensitive. This is not to say that WIMPs are no longer viable dark matter candidates, but rather that it is in our best interest to explore other candidates as well. WIMPs are assumed to have thermalized with the thermal bath in the early universe, although this does not need to be true. Dark matter could have never thermalized with the bath and the observed abundance could still be produced.

Another viable class of dark matter candidates are Feebly Interacting Massive Particles (FIMPs) [53,54] which interact with Standard Model particles so weakly that they do not thermalize with the thermal bath. This means that FIMPs, unlike WIMPs, are not produced by the conventional freeze-out mechanism. More detail on FIMPs and how they could have been produced is given in Chapter 2. Since FIMPs interact so weakly, they certainly evade current direct detection constraints leaving a vast viable parameter space.

In this thesis, we discuss FIMPs and WIMPs which are produced in the early universe through freeze-in and freeze-out mechanisms respectively. We use a benchmark model where dark matter is a Dirac fermion and it interacts with Standard Model fermions through a $Z^{\prime}$ portal in order to explore the two production mechanisms and the boundary between them. We are interested in studying how each production mechanism affects the relic abundance, if the observed relic abundance can be explained by each of these mechanisms, and the regimes of the parameter space where each mechanism dominates. 


\section{Chapter 2}

\section{Dark Matter Production}

\section{Mechanisms}

In this chapter we describe different ways that dark matter could have been produced in the early universe. As discussed in Chapter 1, the abundance of dark matter is known from measurements, but how and when it came to be is unknown. This leaves a massive set of possible dark matter masses and couplings that all lead to the observed final relic density. Different dark matter searches probe particular regions of this dark matter parameter space, so understanding the production of dark matter can motivate current and future experiments. We focus on freeze-out and freeze-in for this thesis, although we briefly discuss alternative mechanisms in this Chapter.

\subsection{Freeze-Out Mechanism}

Particle species that were once part of the thermal bath had to depart from thermal equilibrium and freeze-out in order to have a non-negligible abundance today [55], such as photons, neutrinos and dark matter. From the CMB, there is evidence that 


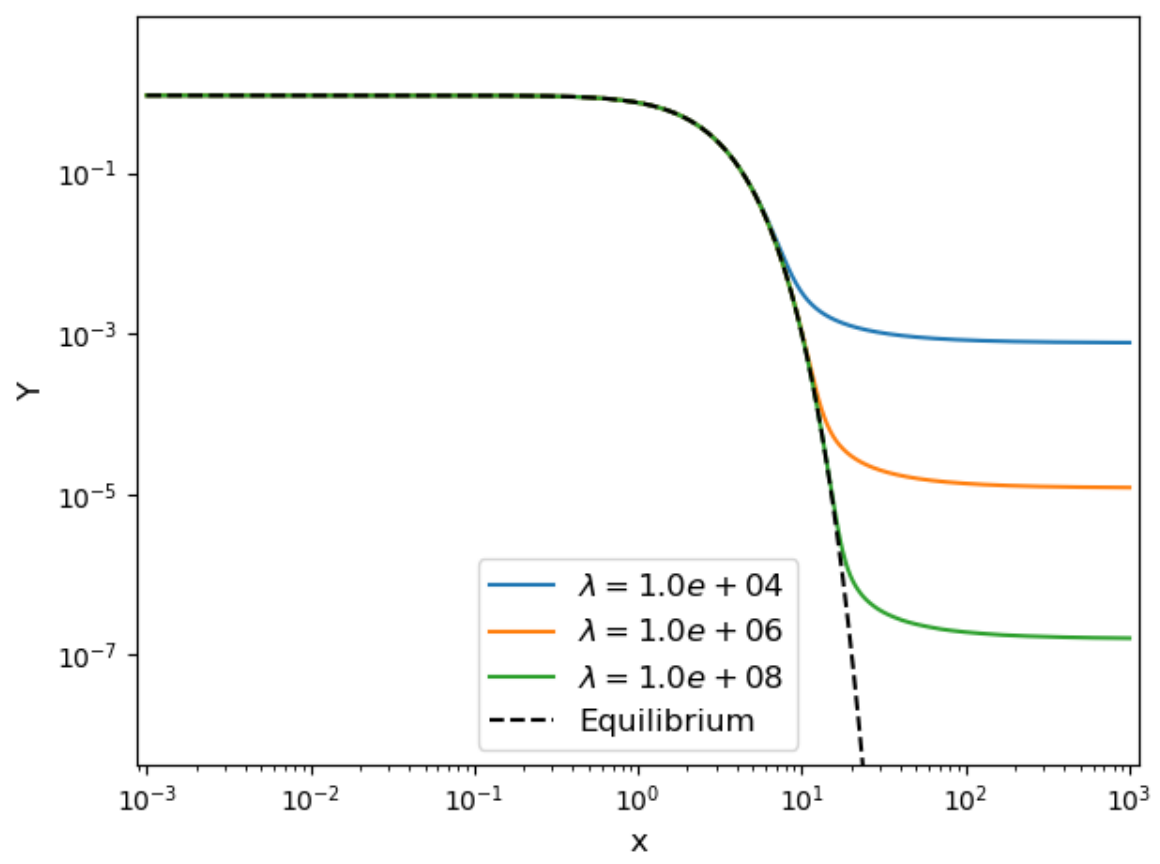

Figure 2.1: The comoving number density as a function of $x \equiv \frac{m}{T}$ where $m$ is the dark matter mass for the freeze-out scenario, plotted for a generic constant cross section at different couplings $(\lambda)$, represented by coloured lines. The dashed black line is the equilibrium yield assuming Maxwell-Boltzmann statistics.

in the early universe photons, electrons, and positrons were once all in thermal equilibrium, comprising an equilibrium thermal bath with a common temperature $T$. A schematic of the freeze-out mechanism is shown in Figure 2.1 where the yield of dark matter (number density per comoving volume, $Y=\frac{n}{s}$ where $n$ is the number density and $s$ is the entropy density) is plotted as a function of $x=\frac{m}{T}$, where $m$ is the mass of the dark matter particle, for different couplings or interaction strengths $\lambda$. More detail is provided in Chapter 3 describing these concepts such as yield and number density. Dark matter produced through freeze-out must have a large enough coupling with standard model particles in order to thermalize with the bath, but not so large that it all annihilates away to nothing as the universe cools. In order for dark matter to thermalize with the bath, the interaction rate between dark matter and bath 
particles must be greater than the Hubble rate, meaning that energy is exchanged efficiently and that they are producing each other. If this thermalization condition is met, dark matter stays in equilibrium until the universe's expansion takes over against the frequency of interactions. This decoupling between dark matter and the thermal bath occurs when approximately no dark matter is being created or destroyed within a Hubble time so its abundance remains constant. This is the "freeze-out" of dark matter particles. If dark matter is more massive than the standard model particles it interacts with, then when the temperature cools to the dark matter mass, production is no longer energetically favourable. This causes the number density of dark matter to drop exponentially since dark matter annihilation is much more likely than production. Dark matter particles remain in equilibrium, annihilating away until the they cannot effectively find each other anymore due to the expansion of the universe. As illustrated by Figure 2.1, the amount of time dark matter tracks the equilibrium curve depends on the coupling. For larger couplings, the interaction rates are larger so that annihilations can occur for longer before the universe's expansion causes the yield to flat line. Therefore the couplings are inversely proportional to the final abundance. As mentioned in Chapter 1, the dark matter candidate that attempts to account for the observed abundance in this way is the so called WIMP.

\subsection{Freeze-In Mechanism}

In contrast to thermally produced dark matter, there are non-thermal methods of production $[56,57]$. If the rate of production of dark matter is low enough, dark matter will not thermalize with the bath and it will be produced through the freeze-in mechanism $[58,59]$. Dark matter production is assumed to begin at the reheating 
temperature $^{3}, T_{R}$, and the abundance of dark matter increases due to its production until the temperature drops so low that these production interactions are kinematically unlikely. Figure 2.2 illustrates the freeze-in mechanism with a plot of the yield as a function of $x$ for different couplings. More detail is given about the freeze-in

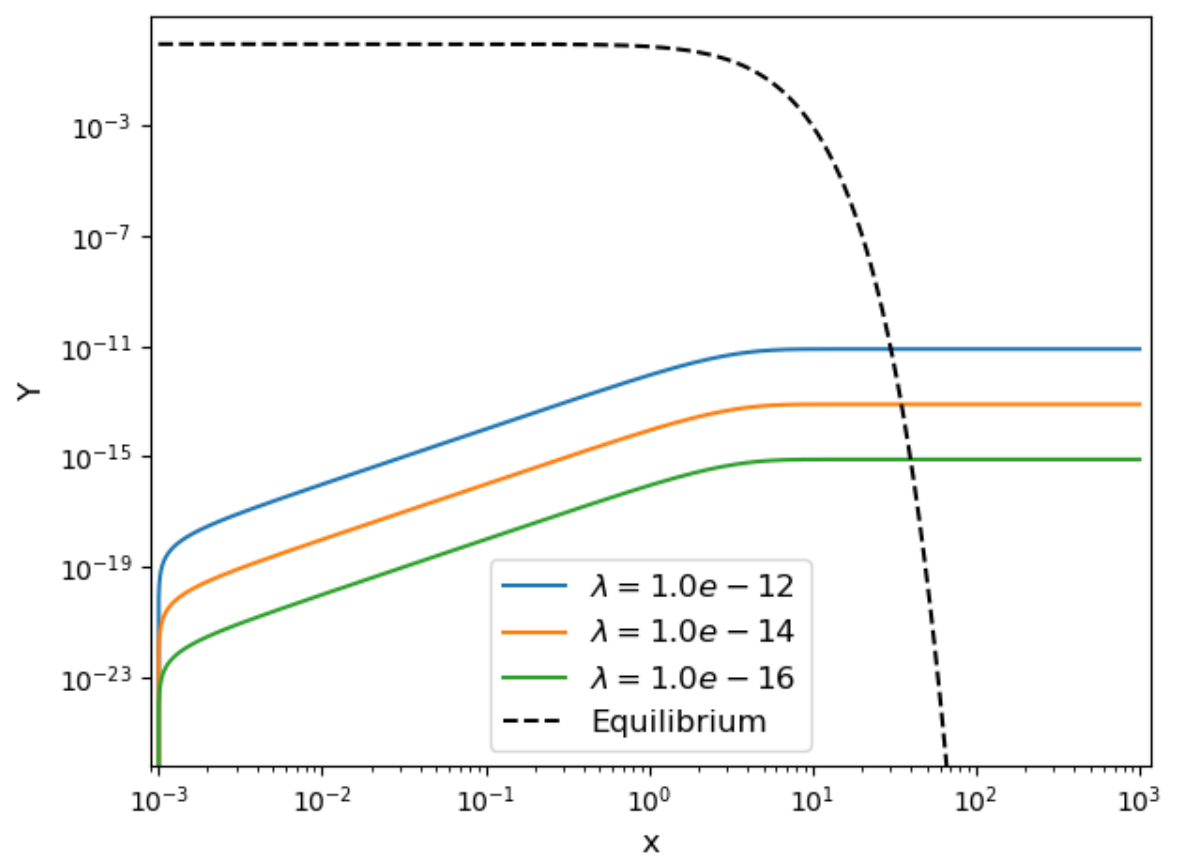

Figure 2.2: The comoving number density as a function of $x \equiv \frac{m}{T}$ where $m$ is the dark matter mass for the freeze-in scenario. Plotted for a generic constant cross section at different couplings $(\lambda)$, represented by coloured lines. The dashed black line is the equilibrium yield assuming Maxwell-Boltzmann statistics. Notice that the yield of dark matter is much less than the equilibrium yield before freeze-in occurs.

mechanism in Chapter 3. Notice that the equilibrium curve (dashed line) is much greater than the dark matter yield before freeze-in up until when the yield flat lines. If dark matter is more massive than the particles it interacts with, the $2 \rightarrow 2$ production processes stop when the temperature is approximately equal to the dark matter mass. If there is a species in the thermal bath that decays into dark matter, the

\footnotetext{
${ }^{3}$ Reheating is the final stage of inflation, when the inflaton (the field responsible for driving inflation) decays into Standard Model particles, transferring its energy [60,61].
} 
rate of production from the decay drops near zero when the temperature becomes less than the mass of the decaying species since the number density of the decaying species exponentially decreases at this point. This is due to the fact that the temperature is so low that production of the decaying particle is no longer probable, therefore it all decays and/or annihilates away. Opposed to the freeze-out scenario, the final abundance is proportional to the coupling since stronger interactions lead to more dark matter being produced. The dark matter candidate that is produced in this way is the FIMP.

\subsection{Other Mechanisms}

There exist other dark matter production mechanisms in the literature that are worth mentioning such as dark freeze-out [53,59,62-65], reannihilation [53,57,59, 62-64,6668], and non-thermal production of various candidates [56], as we discuss in more detail in this section.

\subsubsection{Dark Freeze-Out}

In this scenario, dark matter is initially produced through the freeze-in mechanism ${ }^{4}$ and due to dark sector particles thermalizing with themselves but not with the visible sector, dark matter is produced as a result of freeze-out within the dark sector $[53,59,63]$. For certain parameters, the final abundance is determined by a freeze-out mechanism within the dark sector, not by the initial freeze-in. Figure 2.3 demonstrates the evolution of two dark sector particles in two scenarios. We will call species 1 (blue) the species that thermalizes within the dark sector and species

\footnotetext{
${ }^{4}$ Instead of dark matter being initially populated by freeze-in, it is possible that it was produced by reheating $[53,59,62,69]$.
} 


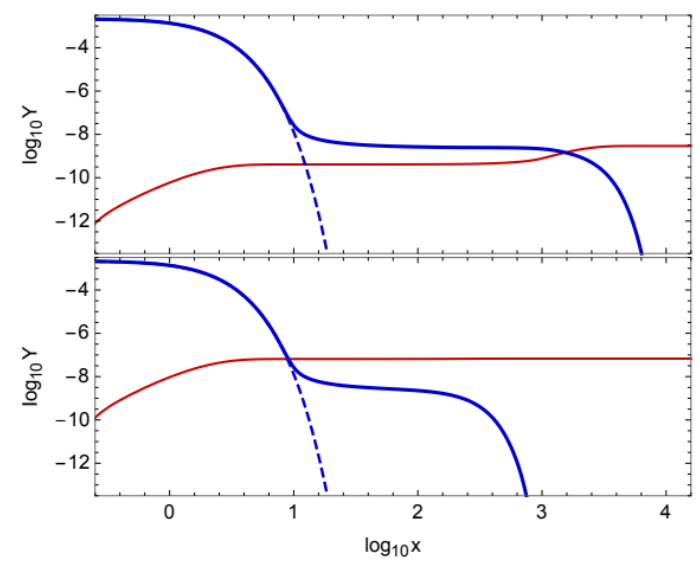

Figure 2.3: Two scenarios of the evolution of two dark sector species. Species 1 thermalizes within the dark sector (which is represented in blue) and species 2 does not thermalize and freezes-in (which is represented in red). The yield $Y \equiv \frac{n}{s}$ is plotted as a function of $x \equiv \frac{m_{1}}{T}$. The dashed blue curves are the equilibrium curves of species 1. This figure comes from Ref. [63].

2 (red) the species that does not, but is affected by this dark freeze-out. The top subfigure shows the scenario where the final abundance of species 2 is determined by the freeze-out of species 1, since as the latter's abundance becomes Boltzmann suppressed it decays and/or annihilates producing species 2. The bottom subfigure shows the case where the initial freeze-in of species 2 dominates, so the abundance from the freeze-out of species 1 is negligible. These scenarios highlight the fact that it can be important to consider thermalization within the dark sector.

\subsubsection{Reannihilation}

Similarly to the dark freeze-out mechanism, in this scenario dark matter is produced as a result of thermalization within the dark sector. Reannihilation begins similarly to dark freeze-out, but dark matter decouples from the other dark sector particles while production from the visible sector is still happening $[53,59]$. As depicted in Figure 2.4, the dark matter yield increases due to production from the visible sector, 


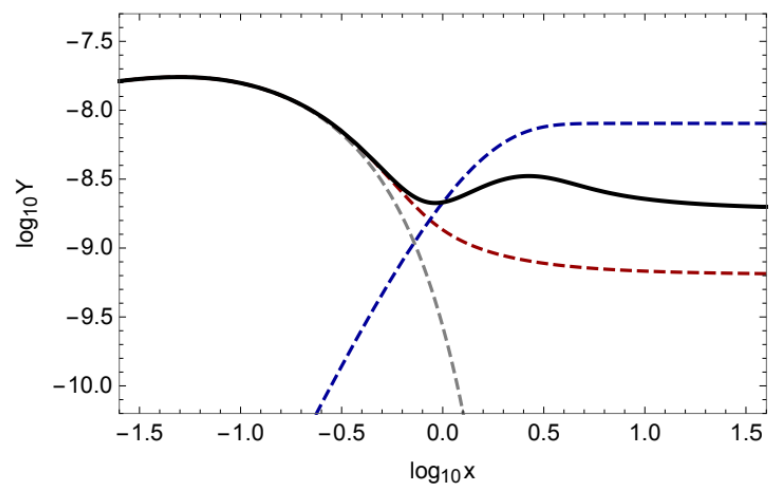

Figure 2.4: The evolution of dark matter in the case of reannihilation (in black), freeze-in (in dashed blue), and freeze-out (in dashed red). The yield $Y \equiv \frac{n}{s}$ is plotted as a function of $x \equiv \frac{m}{T}$. The equilibrium yield is plotted in dashed grey. This figure is from Ref. [53].

until the annihilations to dark sector particles dominate the process and the dark matter yield decreases. The dark matter finally freezes-out for the second time when the expansion of the universe takes over.

\subsubsection{Non-Thermal Candidates}

There are dark matter candidates that have been proposed to be produced nonthermally, that differ from what we have described so far. These other candidates are not the goal of this thesis and are outside of my expertise, but we will mention them for completeness.

The WIMPZILLA is a dark matter candidate that is weakly interacting and has an extremely large mass on the order of $10^{13} \mathrm{GeV}[70,71]$. Since the mass of these particles is so large, they must not have ever been in thermal equilibrium with the bath or else the final abundance would exceed observations. This means that WIMPZILLAs are produced non-thermally either through freeze-in [72], or at the end of inflation gravitationally [73], during reheating [74], during preheating [61, 75-77], 
or in bubble collisions [78].

Axions are an attempt to address the strong $\mathrm{CP}$ problem and can also be a viable dark matter candidate [38]. Axions can be produced thermally [79] although to be able to account for the observed dark matter abundance they must be produced non-thermally. Some proposed production mechanisms are vacuum realignment [80], string decays [81], and domain wall decays [82].

As mentioned earlier, sterile neutrinos are a natural proposed dark matter candidate [27]. These hypothetical neutrinos are similar to the Standard Model ones although are heavier and have weaker interactions. In the early universe they could have been produced through the freeze-out or freeze-in mechanism, or non-thermally by the decay of heavier particles.

Another non-thermal dark matter candidate is the oscillating scalar field [8385]. This scalar field oscillates about the minimum of its potential and behaves as non-relativistic matter that can account for dark matter.

The possibility of very light dark matter with $m \sim 10^{-22} \mathrm{eV}$, the lower bound on the dark matter mass from quantum mechanics and observations, is explored in the literature and often called fuzzy or wave dark matter [86, 87]. The proposed ways of producing these candidates are through a vacuum misalignment in the early universe or decays of cosmic strings [88]. This candidate has interesting consequences for potential observations of galaxy formation.

The inflaton could account for the dark matter in the universe if it only decays during inflation, and remains stable afterwards [89].

There are other proposed dark matter candidates that could have been produced non-thermally in the early universe through a number of mechanisms [56]. We wish to highlight in this section that there is a very long list of diverse and completely rea- 
sonable dark matter candidates that differ from the conventional thermally produced WIMP. 


\section{Chapter 3}

\section{Computing the Dark Matter}

\section{Abundance: The Boltzmann \\ Equation}

Numerous crucial phenomena in cosmology are a result of deviations from thermal equilibrium as the universe evolves, such as inflation, galaxy formation, photon decoupling (cosmic microwave background), and more. Dark matter is the result of nonequilibrium physics. In fact, if dark matter were in equilibrium today its abundance would be nearly zero since it would have all annihilated away. In order to calculate the abundance of dark matter in the universe as a function of temperature given a specific set of production and annihilation processes, we must solve the Boltzmann equation. Throughout this work, we will use natural units where $c=k_{B}=\hbar=1$. 


\subsection{Equilibrium Thermodynamics}

We begin with a brief introduction to equilibrium thermodynamics. The number

density $n_{i}$, the energy density $\rho_{i}$, and the pressure $P_{i}$ of a species $i$ are given by [90],

$$
\begin{gathered}
n_{i}=\frac{\gamma_{i}}{(2 \pi)^{3}} \int f_{i}\left(\overrightarrow{p_{i}}\right) d^{3} p_{i} \\
\rho_{i}=\frac{\gamma_{i}}{(2 \pi)^{3}} \int E_{i}\left(\vec{p}_{i}\right) f_{i}\left(\vec{p}_{i}\right) d^{3} p_{i} \\
P_{i}=\frac{\gamma_{i}}{(2 \pi)^{3}} \int \frac{\left|\overrightarrow{p_{i}}\right|}{3 E_{i}\left(\vec{p}_{i}\right)} f_{i}\left(\overrightarrow{p_{i}}\right) d^{3} p_{i}
\end{gathered}
$$

where $\gamma_{i}$ is the number of internal degrees of freedom, $f_{i}$ is the phase space density, $E_{i}$ is the energy, and $p_{i}$ is the momentum of species $i$. These equations are for a dilute, weakly interacting gas which is a good approximation of what the early universe looked like [90]. The species are in kinetic equilibrium if the particles exchange energy and momentum efficiently through scattering. If this is the case the entropy is maximized so $f_{i}$ is described by equilibrium Fermi-Dirac or Bose-Einstein statistics,

$$
f_{i}^{e q}=\frac{1}{e^{\frac{E-\mu}{T}} \pm 1}
$$

where for Fermi-Dirac we use +1 , for Bose-Einstein we use $-1, \mu$ is the chemical potential, and $T$ is the temperature of the thermal bath. The +1 for fermions comes from the fact that only one fermion can occupy a given state due to the Pauli Exclusion Principle. The -1 for bosons comes from any number of bosons being able to occupy the same state. For thermal bath particles we will neglect the \pm 1 factor assuming Maxwell-Boltzmann statistics and we will also assume zero chemical potential since $\left|\mu_{i}\right| \ll T$ [90]. If the rate of annihilation and production are equal, the particles are 
said to be in chemical equilibrium. For the process $12 \leftrightarrow 34$ in chemical equilibrium, where 1 and 2 label species in the initial state, and 3 and 4 label species in the final state, we have $\mu_{1}+\mu_{2}=\mu_{3}+\mu_{4}$. Species in kinetic and chemical equilibrium will also thermalize, reaching local thermal equilibrium. This comes from the thermodynamic identity,

$$
d U=T d S-P d V+\mu d N
$$

where $N$ is the number of particles, $U$ is the internal energy, $S$ is the total entropy, $V$ is the volume, and in a constant volume $d V=0$. Kinetic equilibrium leads to maximized entropy which implies that $d S=0$, and chemical equilibrium leads to $d N=0$. Therefore we arrive at $d U=0$ which means thermal equilibrium.

In the relativistic case, where $E_{i} \gg m_{i}$ the equilibrium number density $n_{i}^{e q}$ is given by

$$
n_{i}^{e q}= \begin{cases}\frac{\gamma_{i} \zeta(3) T^{3}}{\pi^{2}} & \text { Bosons } \\ \frac{3 \gamma_{i} \zeta(3) T^{3}}{4 \pi^{2}} & \text { Fermions }\end{cases}
$$

where $\zeta$ is the Riemann Zeta function. For the nonrelativistic case, where $m_{i} \gg T$, the \pm 1 term in the distribution function is negligible thus approximately MaxwellBoltzmann so the equilibrium number density is the same for both fermions and bosons. Under this assumption the equilibrium number density is,

$$
n_{i}^{e q}=\frac{\gamma_{i} m_{i}^{2} T K_{2}\left(\frac{m_{i}}{T}\right)}{2 \pi^{2}}
$$

where $K_{j}$ is the modified Bessel function of the second kind of order $j$. For $T \ll m_{i}$ we can then write $n_{i}^{e q}$ as,

$$
n_{i}^{e q} \approx \gamma_{i}\left(\frac{m_{i} T}{2 \pi}\right)^{\frac{3}{2}} e^{\frac{-m_{i}}{T}}
$$


The Hubble parameter dictates the expansion rate of the universe and is defined by,

$$
H \equiv \frac{\dot{a}}{a}
$$

where $a$ is the scale factor. During the radiation dominated era the Hubble parameter is given by,

$$
H(T)=\sqrt{\frac{8 \pi^{3}}{90}} \frac{\sqrt{g_{* e}} T^{2}}{m_{P l}},
$$

where $g_{* e}(T)$ is the effective number of degrees of freedom associated with energy [91] given by,

$$
g_{* e}=\sum_{\text {bosons }} \gamma_{i}\left(\frac{T_{i}}{T}\right)^{4}+\frac{7}{8} \sum_{\text {fermions }} \gamma_{i}\left(\frac{T_{i}}{T}\right)^{4}
$$

where $T_{i}$ is the temperature of species $i$, and $m_{P l}$ is the Planck mass given by $m_{P l}=$ $\sqrt{\frac{1}{G}} \approx 1.22091 \times 10^{19} \mathrm{GeV}$ where $G$ is the gravitational constant. The entropy density $s$ is defined by,

$$
s \equiv \frac{S}{a^{3}}
$$

where $S$ is the total entropy in a comoving volume. The entropy density $s$ is dominated by relativistic species and is given by [90]

$$
s=\frac{2 \pi^{2} g_{* s}(T) T^{3}}{45}
$$

where $g_{* s}$ is the effective number of degrees of freedom associated with entropy given by,

$$
g_{* s}=\sum_{\text {bosons }} \gamma_{i}\left(\frac{T_{i}}{T}\right)^{3}+\frac{7}{8} \sum_{\text {fermions }} \gamma_{i}\left(\frac{T_{i}}{T}\right)^{3} .
$$

The effective number of degrees of freedom are calculated assuming the Standard Model particle content. 


\subsection{The Boltzmann Equation}

For particles that are not in thermal equilibrium we need to solve the Boltzmann equation to describe the evolution of the number density. The equation governing the evolution of the number density of particle $3^{5}$ is given by $[90]^{6}$,

$$
\dot{n}_{3}+3 H n_{3}=R_{3}(t)
$$

where $R$ is the interaction rate density,

$$
R_{3}=\frac{\gamma_{3}}{(2 \pi)^{3}} \int C \frac{d^{3} p_{3}}{E_{3}}
$$

and $C$ is the collision operator containing all number changing interactions acting on the distribution function of species 3. The rate density (or collision term) for the generic process $i+j+\cdots \leftrightarrow k+l+\cdots$ is given by [90,92],

$$
\begin{gathered}
\frac{\gamma_{i}}{(2 \pi)^{3}} \int C \frac{d^{3} p_{i}}{E_{i}}=\int d \Pi_{i} d \Pi_{j} \cdots d \Pi_{k} d \Pi_{l} \cdots(2 \pi)^{4} \delta^{4}\left(p_{i}+p_{j}+\cdots-p_{k}-p_{l}-\cdots\right) \\
{\left[|\bar{M}|_{k+l+\cdots \rightarrow i+j+\cdots}^{2} f_{k} f_{l} \cdots\left(1 \pm f_{i}\right)\left(1 \pm f_{j}\right) \cdots\right.} \\
\left.-|\bar{M}|_{i+j+\cdots \rightarrow k+l+\cdots} f_{i} f_{j} \cdots\left(1 \pm f_{k}\right)\left(1 \pm f_{l}\right) \cdots\right]
\end{gathered}
$$

where $|\bar{M}|^{2}$ is the squared amplitude averaged over initial and final spins ${ }^{7}$ for the process calculated using quantum field theory, $d \Pi_{i}$ are the phase space differential

\footnotetext{
${ }^{5}$ We will label the particle of interest (dark matter) as particle 3 since the production interactions involved will be labeled $12 \rightarrow 34$ and $1 \rightarrow 34$ (and reverse for dark matter annihilation) where 3 and 4 are dark matter and 1 and 2 are species in the thermal bath.

${ }^{6}$ Equation 3.15 is actually the Boltzmann equation integrated over the momenta or the Boltzmann fluid equation, since in this work we are interested in the macroscopic evolution of particles.

${ }^{7}$ In cosmology we average over initial and final spins, rather than averaging over initial and summing over final spins, since we do not know the initial or final states.
} 
elements,

$$
d \Pi_{i}=\frac{\gamma_{i} d^{3} p_{i}}{(2 \pi)^{3} 2 E_{i}}
$$

and the $(1 \pm f)$ factors are the stimulated $(+)$ and blocking $(-)$ factors for final state bosons and fermions respectively.

It is useful to define the yield $Y$, the number density in a comoving volume, to scale out the expansion of the universe,

$$
Y \equiv \frac{n}{s}
$$

We will also define the parameter $x$ which acts as a time variable,

$$
x \equiv \frac{m}{T}
$$

where we take $m$ to be the mass of the particle we're interested in (in this case $m_{3}$ ). We can now write the Boltzmann equation as a function of $Y$ rather than $n$,

$$
\dot{n}+3 H n=\dot{Y} s+Y \dot{s}+3 \frac{\dot{a}}{a} Y s
$$

since $H \equiv \frac{\dot{a}}{a}$ and by assuming the total entropy per comoving volume is conserved, $S=s a^{3}=c$, where $c$ is a constant,

$$
\dot{n}+3 H n=\dot{Y} s+Y \frac{-3 \dot{a} c}{a^{4}}+3 \frac{\dot{a}}{a} Y \frac{c}{a^{3}}=\dot{Y} s
$$

We do a change of variables from $t$ to $x$,

$$
\frac{d Y}{d x}=\frac{d Y}{d a} \frac{d a}{d x}=\frac{d Y}{d t} \frac{d t}{d a} \frac{d a}{d x}=\dot{Y} \frac{1}{\dot{a}} \frac{d}{d x}\left(\frac{c^{\frac{1}{3}}}{s^{\frac{1}{3}}}\right)=-\frac{\dot{Y}}{3 H s} \frac{d s}{d x}
$$


where the derivative of $s$ with respect to $x$ is,

$$
\frac{d s}{d x}=\frac{d s}{d T} \frac{d T}{d x}=-\frac{3 s T}{m}\left(1+\frac{T}{3 g_{* s}} \frac{d g_{* s}}{d T}\right)
$$

Defining $g_{*}$ as,

$$
g_{*} \equiv \frac{g_{* s}}{\sqrt{g_{* e}}}\left(1+\frac{T}{3 g_{* s}} \frac{d g_{* s}}{d T}\right)
$$

and $\tilde{g}_{*}$ as,

$$
\tilde{g_{*}} \equiv\left(1+\frac{T}{3 g_{* s}} \frac{d g_{* s}}{d T}\right)
$$

hence,

$$
\frac{d Y_{3}}{d x}=\frac{\dot{Y}_{3} \tilde{g}_{*}}{H x}
$$

Now we have the Boltzmann fluid equation in terms of the yield $Y$ and the convenient variable $x$ that goes in the same direction as time.

\subsection{Freeze-Out}

The freeze-out mechanism is described in section 2.1, but in this section we show how to compute the dark matter abundance in the freeze-out regime. For the case of freeze-out, the dark matter is in equilibrium with the thermal bath until the universe's expansion dominates over the frequency of interactions, more specifically the dark matter particles can no longer find each other to interact. This occurs when the Hubble rate becomes larger than the interaction rates. 


\subsubsection{Boltzmann Equation for $12 \leftrightarrow 34$}

After changing variables from $T$ to $x$ and from $n$ to $Y$ for the process $12 \leftrightarrow 34$, the Boltzmann equation, equation 3.15, becomes $^{8}$,

$$
\frac{d Y_{3}}{d x}=\frac{\tilde{g}_{*}}{H s x} S_{f} \int d \Pi_{1} d \Pi_{2} d \Pi_{3} d \Pi_{4}(2 \pi)^{4}|\bar{M}|_{12 \rightarrow 34}^{2} \delta^{4}\left(p_{1}+p_{2}-p_{3}-p_{4}\right)\left(f_{1} f_{2}-f_{3} f_{4}\right)
$$

where $S_{f}$ is a statistical factor accounting for identical particles where for identical particles in the final or initial state there is a factor $\frac{1}{n !}$. A useful relation that relates the integral in equation 3.28 to the scattering angle $\theta$ is given by,

$$
\int d \Pi_{i} d \Pi_{j} F\left(\vec{p}_{i}, \overrightarrow{p_{j}}, \theta\right)(2 \pi)^{4} \delta^{4}\left(p_{i}+p_{j}-p\right)=\frac{1}{8} \frac{\left|p_{i j}\right|}{\sqrt{s_{m}}} \int F\left(s_{m}, \theta\right) d \Omega
$$

where $d \Omega=\sin \theta d \theta d \phi, p=p_{1}$ for a decay and $p=p_{k}+p_{l}$ for $2 \rightarrow 2, F$ is any function of the momentum which for our purposes is the squared amplitude, and $s_{m}=\left(p_{1}+p_{2}\right)^{2}$ is the Mandelstam variable.

Since we assume Maxwell-Boltzmann statistics for all particle species rather than Fermi-Dirac and Bose-Einstein statistics the phase space density for bath particles are given by,

$$
f_{i}=e^{-E_{i} / T}
$$

We can rewrite equation 3.28 in terms of the thermally averaged annihilation cross section $<\sigma v>_{i j \rightarrow k l}[90]$,

$$
<\sigma v>_{i j \rightarrow k l} \equiv \frac{S_{f}}{n_{i}^{e q} n_{j}^{e q}} \int d \Pi_{1} d \Pi_{2} d \Pi_{3} d \Pi_{4}(2 \pi)^{4}|\bar{M}|_{k l \rightarrow i j}^{2} \delta^{4}\left(p_{k}+p_{l}-p_{i}-p_{j}\right) f_{i}^{e q} f_{j}^{e q}
$$

\footnotetext{
${ }^{8}$ We are omitting the $1 \pm f$ blocking and stimulated emission factors, and we are also assuming charge-parity invariance (or time invariance) thus $|\bar{M}|_{12 \rightarrow 34}^{2}=|\bar{M}|_{34 \rightarrow 12}^{2}$.
} 
In the freeze-out regime we can safely make the assumption that particle 3 is in thermal equilibrium until it decouples. Since for freeze-out the calculations will be with lower temperatures we can assume a Maxwell-Boltzmann distribution to characterize the equilibrium number density of particle 3 . Whenever particle 3 is in kinetic equilibrium during chemical decoupling we can relate the number density $n$ to the phase space density $f$ by [93],

$$
f_{i}=f_{i}^{e q} \frac{n_{i}}{n_{i}^{e q}}
$$

Using equation 3.32 we get,

$$
\frac{d Y_{3}}{d x}=\frac{\tilde{g}_{*}}{H s x}<\sigma v>_{34 \rightarrow 12}\left(n_{3}^{e q} n_{4}^{e q}-n_{3} n_{4}\right)
$$

We now have a differential equation for a given process $12 \rightarrow 34$ that we can solve numerically for yield $Y$. If particle 3 and 4 are the same (or the antiparticle of each other) then,

$$
\frac{d Y_{3}}{d x}=\sqrt{\frac{\pi}{45}} \frac{m_{P l} m_{3} g_{*}}{x^{2}}<\sigma v>_{34 \rightarrow 12}\left(\left(Y_{3}^{e q}\right)^{2}-Y_{3}^{2}\right)
$$

where $Y^{e q}$ is the equilibrium yield given by,

$$
Y_{i}^{e q}=\frac{n_{i}^{e q}}{s}=\frac{45 \gamma_{i} x^{2} K_{2}(x)}{4 \pi^{4} g_{* s}}
$$

where $n_{i}^{e q}$ and $s$ is given in equations 3.7 and 3.13 respectively.

\subsubsection{Boltzmann Equation for $1 \leftrightarrow 34$}

For the process $1 \leftrightarrow 34$ we can write the Boltzmann equation as,

$$
\frac{d Y_{3}}{d x}=\frac{\tilde{g}_{*}}{H s x} S_{f} \int d \Pi_{1} d \Pi_{3} d \Pi_{4}(2 \pi)^{4}|\bar{M}|_{1 \rightarrow 34}^{2} \delta^{4}\left(p_{1}-p_{3}-p_{4}\right)\left(f_{1}-f_{3} f_{4}\right) .
$$


We can write this equation in terms of the decay rate defined by,

$$
\Gamma_{1 \rightarrow 34}=\frac{S_{f}}{2 m_{1}} \int|\bar{M}|^{2}(2 \pi)^{4} \delta^{4}\left(p_{1}-p_{3}-p_{4}\right) d \Pi_{3} d \Pi_{4}
$$

Equation 3.36 becomes,

$$
\frac{d Y_{3}}{d x}=\sqrt{\frac{90}{8 \pi^{3}}} \frac{g_{*} m_{P l} x \Gamma_{1 \rightarrow 34}}{m_{3}^{2} s g_{* s}} \int 2 m_{1} d \Pi_{1}\left(f_{1}-f_{3} f_{4}\right) .
$$

Similar to the $12 \leftrightarrow 34$ case, using equation 3.32 and conservation of energy we arrive at,

$$
f_{1}-f_{3} f_{4}=e^{-E_{1} / T}\left(1-\frac{n_{3} n_{4}}{n_{3}^{e q} n_{4}^{e q}}\right) .
$$

Substituting this into equation 3.38,

$$
\frac{d Y_{3}}{d x}=\sqrt{\frac{90}{8 \pi^{3}}} \frac{g_{*} m_{P l} x \Gamma_{1 \rightarrow 34}}{m_{3}^{2} s g_{* s}(2 \pi)^{3}}\left(1-\frac{n_{3} n_{4}}{n_{3}^{e q} n_{4}^{e q}}\right) \int \frac{m_{1} \gamma_{1}}{E_{1}} d^{3} p_{1} e^{-E_{1} / T}
$$

Changing the variable of integration to energy, rearranging, and using the relation,

$$
\int_{m}^{\infty} \sqrt{E^{2}-m^{2}} e^{-E / T} d E=m T K_{1}\left(\frac{m}{T}\right)=\frac{2 \pi^{2}}{m \gamma} n^{e q} \frac{K_{1}\left(\frac{m}{T}\right)}{K_{2}\left(\frac{m}{T}\right)}
$$

where $K_{1}\left(\frac{m}{T}\right) \approx K_{2}\left(\frac{m}{T}\right)$ for $m>T$ so we will just take the ratio $\frac{K_{1}\left(\frac{m}{T}\right)}{K_{2}\left(\frac{m}{T}\right)} \approx 1$. For identical species 3 and 4 we get,

$$
\frac{d Y_{3}}{d x}=\sqrt{\frac{\pi}{45}} \frac{n_{1}^{e q} g_{*} m_{P l} \Gamma_{1 \rightarrow 34}}{m_{3}^{5} \gamma_{3}^{2} K_{2}(x)^{2}}\left(\left(Y_{3}^{e q}\right)^{2}-Y_{3}^{2}\right)
$$

Now we have differential equations we can use to solve for the yield $Y$ for both $1 \leftrightarrow 34$ and $12 \leftrightarrow 34$ processes. When solving the Boltzmann equation for multiple 
processes, each process will simply be added. For example, if there exists two $12 \leftrightarrow 34$ processes that we will label $i j \leftrightarrow 34$ and $k l \leftrightarrow 34$, and one $1 \leftrightarrow 34$ process we obtain,

$$
\begin{aligned}
\frac{d Y_{3}}{d x}= & m_{P l} \sqrt{\frac{\pi}{45}}\left(\frac{n_{1}^{e q} g_{*} \Gamma_{1 \rightarrow 34}}{m_{3}^{5} \gamma_{3}^{2} K_{2}(x)^{2}}+\frac{m_{3} g_{*}}{x^{2}}\left(<\sigma v>_{34 \rightarrow i j}+<\sigma v>_{34 \rightarrow k l}\right)\right) \\
& \times\left(\left(Y_{3}^{e q}(x)\right)^{2}-Y_{3}^{2}\right) .
\end{aligned}
$$

\subsubsection{Freeze-Out Approximation}

If we know the freeze-out temperature $T_{f}$, we can approximate the final yield. To approximately calculate the freeze-out temperature (or $x_{f}=\frac{m_{3}}{T_{f}}$ ) we first write the Boltzmann equation in terms of $\Delta[92]$,

$$
\Delta \equiv Y_{3}-Y_{3}^{e q}
$$

for the $12 \rightarrow 34$ process,

$$
\frac{d \Delta}{d x}=-\sqrt{\frac{\pi}{45}} \frac{m_{P l} m_{3} g_{*}}{x^{2}}<\sigma v>_{34 \rightarrow 12}\left(\Delta^{2}+2 \Delta Y^{e q}\right)-\frac{d Y_{3}^{e q}}{d x} .
$$

At high temperatures $\Delta=0$ since dark matter is in equilibrium but when dark matter freezes out the yield starts to deviate from its equilibrium value. Thus when freeze

out occurs $\Delta=\delta Y_{3}^{e q}$, where $\delta$ is taken to be 1.5 [92]. Before freeze-out, $\frac{d \Delta}{d x} \approx 0$ since $Y_{3} \approx Y_{3}^{e q}$. Then we have an equation we can numerically solve for $x_{f}$,

$$
\left.\frac{d Y_{3}^{e q}}{d x}\right|_{x_{f}} \approx-\sqrt{\frac{\pi}{45}} \frac{m_{P l} m_{3} g_{*}\left(x_{f}\right)}{x_{f}^{2}}<\sigma v>_{34 \rightarrow 12}\left(Y_{3}^{e q}\left(x_{f}\right)\right)^{2}\left(\delta^{2}+2 \delta\right)
$$


where $\left.\frac{d Y_{3}^{e q}}{d x}\right|_{x_{f}}$ is the derivative of $Y_{3}^{e q}$ with respect to $x$ evaluated at $x_{f}$ and $Y^{e q}$ is given by equation 3.35. Similarily for $1 \rightarrow 34$,

$$
\left.\frac{d Y^{e q}}{d x}\right|_{x_{f}} \approx-\sqrt{\frac{\pi}{45}} \frac{n_{1}^{e q} g_{*} m_{P l} \Gamma_{1 \rightarrow 34}}{m_{3}^{5} \gamma_{3}^{2} K_{2}(x)^{2}}\left(Y^{e q}\left(x_{f}\right)\right)^{2}\left(\delta^{2}+2 \delta\right) .
$$

Alternatively, the decoupling temperature can be found by comparing the Hubble rate to the frequency of annihilations $W^{9}$, where $W_{34 \rightarrow 12}=n_{3}^{e q}<\sigma v>_{34 \rightarrow 12}$ and $W_{1 \rightarrow 34}=\frac{n_{1}^{e q}}{n_{3}^{e q}} \Gamma_{1 \rightarrow 34}$ before species 3 decouples. When the Hubble rate is equal to the annihilation frequency, the dark matter decouples from the bath.

We can use this result to calculate an approximate value for the final yield, $Y_{\infty}$. Since after freeze-out $Y^{e q} \ll Y$, we can safely neglect the $Y^{e q}$ term in equations 3.34 and 3.42. We now have a separable differential equation that can be solved by integrating from 0 to $T_{f}$ for $12 \rightarrow 34$,

$$
\frac{d Y}{d x} \approx-\sqrt{\frac{\pi}{45}} \frac{m_{P l} m_{3} g_{*}}{x^{2}}<\sigma v>_{34 \rightarrow 12} Y^{2}
$$

and for $1 \rightarrow 34$,

$$
\frac{d Y}{d x} \approx-\sqrt{\frac{\pi}{45}} \frac{n_{1}^{e q} g_{*} m_{P l} \Gamma_{1 \rightarrow 34}}{m_{3}^{5} \gamma_{3}^{2} K_{2}(x)^{2}} Y^{2}
$$

Integrating both sides and changing variables from $x$ to $T$,

$$
\frac{1}{Y_{\infty}} \approx \frac{1}{Y_{f}}+\int_{0}^{T_{f}} \sqrt{\frac{\pi}{45}} \frac{m_{P l} m_{3} g_{*}}{x^{2}}<\sigma v>_{34 \rightarrow 12} d T .
$$

Similarily for $1 \rightarrow 34$,

$$
\frac{1}{Y_{\infty}} \approx \frac{1}{Y_{f}}+\int_{0}^{T_{f}} \sqrt{\frac{\pi}{45}} \frac{n_{1}^{e q}(T) g_{*} m_{P l} \Gamma_{1 \rightarrow 34}}{m_{3}^{5} \gamma_{3}^{2} K_{2}\left(\frac{m_{3}}{T}\right)^{2}} d T
$$

\footnotetext{
${ }^{9}$ Interaction frequencies and rate densities are discussed in more detail in section 3.5.
} 
where $Y_{\infty}$ is the present yield and $Y_{f} \equiv Y_{3}^{e q}\left(x_{f}\right)(1+\delta)$. This approximation can only be applied if we are in the freeze-out regime. This can only happen if the density of dark matter reaches equilibrium, which occurs if the production rate is high enough for any temperature less than reheating and sufficiently greater than the dark matter mass, since freeze-out occurs at temperatures around the dark matter mass. As mentioned above, for multiple processes the integrand on the right hand side of equations 3.51 and/or 3.50 just sums over each process.

We can approximate the final yield of particle $3, Y_{\infty}$, in the freeze-out scenario for the process $12 \leftrightarrow 34$ by defining a constant $\lambda[94]$,

$$
\lambda=\sqrt{\frac{\pi}{45}} \frac{m_{P l} m_{3} g_{*}}{x^{2}}<\sigma v>_{34 \rightarrow 12}
$$

where we take $\langle\sigma v\rangle_{34 \rightarrow 12}$ and $g_{*}$ to both be constant with temperature just for the sake of this derivation. We now can turn equation 3.34 into the Riccati equation,

$$
\frac{d Y_{3}}{d x}=\frac{\lambda}{x^{2}}\left(\left(Y_{3}^{e q}\right)^{2}-Y_{3}^{2}\right)
$$

where we use the approximations described above to arrive at,

$$
\frac{1}{Y_{\infty}} \approx \frac{1}{Y_{f}}+\frac{\lambda}{x_{f}}
$$

and since $Y_{f} \ll Y_{\infty}$,

$$
Y_{\infty} \approx \frac{x_{f}}{\lambda}
$$

We see from equation 3.55 that the final abundance is inversely proportional to $\lambda$, the interaction rates, since for higher rates more annihilations occur and the equilibrium curve is followed for longer as in Figure 2.1. 


\subsection{Freeze-In Approximation}

Section 2.2 goes over the freeze-in mechanism, although in this section we explain how to compute the dark matter abundance for the freeze-in regime. If the couplings are small enough and/or the mediator mass is large enough such that the interaction rate densities are small enough, the density of the dark matter particles is always much lower than the density of the bath particles, thus dark matter never reaches thermal equilibrium with the rest of the bath. For this case, an approximation can be applied to the Boltzmann equation in order to more easily calculate the relic abundance. If the abundance never reaches thermal equilibrium, we can safely say that the $f_{3} f_{4}$ term in equation 3.28 is negligible compared to the $f_{1} f_{2}$ term hence,

$$
\frac{d Y_{3}}{d x} \approx \sqrt{\frac{90}{8 \pi^{3}}} \frac{S_{f} g_{*} m_{P l} x}{m_{3}^{2} s g_{* s}} \int d \Pi_{1} d \Pi_{2} d \Pi_{3} d \Pi_{4}(2 \pi)^{4}|\bar{M}|_{12 \rightarrow 34}^{2} \delta^{4}\left(p_{1}+p_{2}-p_{3}-p_{4}\right) f_{1} f_{2}
$$

This results in a separable ordinary differential equation, which is solved by integration. Under this approximation we are assuming that only dark matter production is happening, and that the DM annihilations are negligible. Writing this in terms of the thermally averaged production cross section $\langle\sigma v\rangle_{12 \rightarrow 34}$ we then get the equation for the yield $Y$ as a function of $x$ for $12 \rightarrow 34$,

$$
Y_{3}(x) \approx \frac{135 \sqrt{5} m_{P l}}{4 \pi^{3} \sqrt{\pi} m_{3}^{5}} \int_{x_{R}}^{x} \frac{g_{*} x^{\prime 4}}{g_{* s}^{2}}<\sigma v>_{12 \rightarrow 34} n_{1}^{e q} n_{2}^{e q} d x^{\prime}
$$

and for $1 \rightarrow 34$,

$$
Y_{3}(x) \approx \sqrt{\frac{90}{8 \pi^{3}}} \frac{m_{P l}}{m_{3}^{2}} \int_{x_{R}}^{x} \frac{g_{*} x^{\prime}}{g_{* s} s} n_{1}^{e q} \Gamma_{1 \rightarrow 34} d x^{\prime}
$$


where $x_{R}=\frac{m_{\chi}}{T_{R}}$ and $T_{R}$ is the reheating temperature. Notice that we are assuming $Y_{3}\left(x_{R}\right) \approx 0$. Similarly to the freeze-out case, when there are multiple processes involved the right hand side of equations 3.57 and/or 3.58 turns into a sum over each process. We write the yield for freeze-in in terms of the dark matter production cross section because if we were to use the annihilation cross section instead, we would have to make an assumption about the distribution of dark matter. We assume that dark matter obeys Maxwell Boltzmann statistics for freeze-out, but this is acceptable since it is non-relativistic during its decoupling. The freeze-in approximation is only valid under the condition that the production rate is slower than the Hubble rate for all temperatures before Boltzmann suppression. As the temperature decreases, the reaction rate of a process will drop off very quickly at some temperature due to the fact that the average momentum of the particles is too low to undergo a reaction. This Boltzmann suppression effect is from the exponential in the Maxwell-Boltzmann distribution that decreases rapidly as temperature decreases.

\subsection{Interaction Rates}

In the previous section, we derived the equations used to solve for the yield of a species $i$ given a particular process. We can write these equations in terms of the interaction rate density $R_{i}(t)$, which is the number of interactions per unit time and volume. For the $12 \leftrightarrow 34$ process,

$$
R_{3}=R_{12 \rightarrow 34}-R_{34 \rightarrow 12}
$$

with,

$$
R_{i j \rightarrow k l}=n_{i} n_{j}<\sigma v>_{i j \rightarrow k l} .
$$


For the $1 \leftrightarrow 34$ process,

$$
R_{3}=R_{1 \rightarrow 34}-R_{34 \rightarrow 1}
$$

with,

$$
R_{1 \rightarrow 34} \approx n_{1} \Gamma_{1 \rightarrow 34}
$$

and

$$
R_{34 \rightarrow 1} \approx \frac{n_{3} n_{4}}{n_{3}^{e q} n_{4}^{e q}} n_{1} \Gamma_{1 \rightarrow 34}
$$

We can compute $\left\langle\sigma v>_{34 \rightarrow 12}\right.$ using equation 3.31 and the equation for the cross section $\sigma_{34 \rightarrow 12}[92]$,

$$
\sigma_{34 \rightarrow 12}=\frac{S_{f}\left|\overrightarrow{p_{f}}\right|}{\sqrt{s_{m}} 64 \pi^{2}\left|\overrightarrow{p_{i}}\right|} \int \frac{|\bar{M}|_{12 \rightarrow 34}^{2} \delta^{4}\left(p_{3}+p_{4}-p_{1}-p_{2}\right) d^{3} \overrightarrow{p_{1}} d^{3} \overrightarrow{p_{2}}}{\sqrt{\left|\overrightarrow{p_{1}}\right|^{2}+m_{1}^{2}} \sqrt{\left|\overrightarrow{p_{2}}\right|^{2}+m_{2}^{2}}}
$$

where $\left|\overrightarrow{p_{i}}\right|$ and $\left|\overrightarrow{p_{f}}\right|$ are the magnitudes of the initial and final momenta. Taking $m_{1}=m_{2}$ and $m_{3}=m_{4}$ since as we will see in Chapter 4 this is true for our model, the thermally averaged cross section is written as,

$$
<\sigma v>_{34 \rightarrow 12}=\frac{\int \sigma_{34 \rightarrow 12}\left(s_{m}-4 m_{3}^{2}\right) \sqrt{s_{m}} K_{1}\left(\frac{\sqrt{s_{m}}}{T}\right) d s_{m}}{8 m_{3}^{4} T K_{2}\left(\frac{m_{3}}{T}\right)^{2}}
$$

We will also define the frequency of interactions which is usually written as $\Gamma$ but to not confuse this with the decay rate we will call it $W$. $W$ is the time scale of the interactions [94] or more precisely the number of interactions per unit time, defined by,

$$
W_{12 \rightarrow 34} \equiv \frac{R_{12 \rightarrow 34}}{n_{2}}=n_{1}<\sigma v>_{12 \rightarrow 34}
$$

and $W_{1 \rightarrow 34}$,

$$
W_{1 \rightarrow 34} \equiv \frac{R_{1 \rightarrow 34}}{n_{1}} \approx \Gamma_{1 \rightarrow 34}
$$


The frequencies of interactions are important to consider since comparing them to the Hubble rate tells us when the particles of interest decouple from the thermal bath or if they ever even thermalize with the bath. If $W<H$ for all relevant temperatures the production regime is certainly freeze-in since the interactions cannot keep up with the expansion of the universe. Relevant temperatures are all temperatures that are less than the reheating temperature $T_{R}$ since that is when we are assuming dark matter production began [95]. If $W>H$ at some point then equilibrium will be reached and dark matter will remain in equilibrium until it becomes too heavy to be produced and freezes out. This effect comes directly from equation 3.15 where we have $n H$ on one side of the equation and $R$ on the other. This implies that if $H>\frac{R}{n}=W$ the expansion dominates, while if we have $H<\frac{R}{n}=W$ the interactions are frequent enough to thermalize with the thermal bath. 


\section{Chapter 4}

\section{Model}

\subsection{Lagrangian}

Our model consists of a Dirac fermionic dark matter candidate, $\chi$, which interacts with Standard Model fermions through a $Z^{\prime}$ vector boson, the gauge boson of a $U(1)$ extension to the SM symmetry group, $U(1)^{\prime}$. $U(1)^{\prime}$ extensions have been motivated by grand unified theories, string models, and other extensions [96-98]. In our case this extension provides a way for dark matter to interact with the visible sector, through a $Z^{\prime}$ portal.

Since we have two abelian gauge groups ${ }^{10}$, the Standard Model hypercharge $U(1)$ and the $U(1)^{\prime}$, there must exist kinetic mixing between the two gauge bosons given that it is allowed by renormalizability and gauge invariance [99]. The interaction between the visible and dark sectors can occur through kinetic mixing between $U(1)$ and $U(1)^{\prime}$ [100-102]. We are not concerned with how exactly the $Z^{\prime}$ acquired mass, whether through the Higgs or another scalar particle, only with its effect on dark

\footnotetext{
${ }^{10}$ The abelian group is the group where all of the elements commute with each other. The $U(1)$ group is abelian.
} 


\begin{tabular}{c|c|c}
$f$ & $V_{f}$ & $A_{f}$ \\
\hline$\nu$ & 0.1795 & 0.1795 \\
$e, \mu, \tau$ & -0.0134 & -0.1795 \\
$u, c, t$ & 0.0687 & 0.1795 \\
$d, s, b$ & -0.124 & -0.1795
\end{tabular}

Table 4.1: Values of $V_{f}$ and $A_{f}$, the axial and vector $Z^{\prime}$ Standard Model fermion couplings.

matter evolution. If the Standard Model Higgs is charged under this new symmetry, there will also be a mass mixing between the $Z$ and $Z^{\prime}$. Interaction between the Standard Model $Z$ and dark matter can arise if there is mass mixing or even if not, through fermion loops [103]. However, we will assume that there is a negligible effect from $Z^{\prime} / Z$ mixings on the dark matter abundance [104-108].

In our model the $Z^{\prime}$ boson directly couples to dark matter and to Standard Model fermions. The interaction Lagrangian for our model is given by,

$$
\mathcal{L} \supset \bar{\chi} \gamma^{\mu}\left(V_{\chi}-A_{\chi} \gamma_{5}\right) \chi Z_{\mu}^{\prime}+\sum_{i} \bar{f}_{i} \gamma^{\mu}\left(V_{f, i}-A_{f, i} \gamma_{5}\right) f_{i} Z_{\mu}^{\prime}
$$

where $f_{i}$ are the Standard Model fermion fields including leptons and quarks, $\chi$ is the Dirac fermion dark matter field, $Z^{\prime}$ is the mediator field, $A_{f, i}$ and $V_{f, i}$ are the axial and vector couplings between $Z^{\prime}$ and $f$, and $A_{\chi}$ and $V_{\chi}$ are the axial and vector couplings between $\chi$ and $Z^{\prime}$.

We are studying the sequential $Z^{\prime}$ model which has the same couplings to Standard Model fermions as the Standard Model $Z$ boson since it is a useful benchmark model $[104,109,110]$. We recognize that our model is incomplete but we are interested in $A_{\chi}$ and $V_{\chi}$, the couplings between Standard Model particles and dark matter. Table 4.1 shows the values for $A_{f}$ and $V_{f}$ which are calculated using the 
Glashow-Weinberg-Salam (GWS) model couplings $C_{V}^{f}$ and $C_{A}^{f}[111]$ and using,

$$
V_{f}=\frac{g_{Z}}{2} C_{V}^{f}
$$

and

$$
A_{f}=\frac{g_{Z}}{2} C_{A}^{f}
$$

where $g_{Z}$ is the neutral weak coupling constant given by,

$$
g_{Z}=\frac{g_{e}}{\sin \theta_{w} \cos \theta_{w}}=\frac{2 \sqrt{\alpha \pi}}{\sin \theta_{w} \cos \theta_{w}}=0.7180
$$

where $g_{e}$ is the quantum electrodynamics (QED) coupling constant, $\theta_{w}=27.76^{\circ}$ is the weak mixing angle, and $\alpha=\frac{1}{137.036}$ is the fine structure constant. We chose to define the couplings in such a way that absorbs the $g_{Z}$ and $C^{f}$ values for simplicity. This leaves the dark sector couplings $A_{\chi}$ and $V_{\chi}$ and the masses of $Z^{\prime}$ and $\chi$ to be the free parameters of our model.

\subsection{Processes}

When considering the evolution of the number density of dark matter, the only processes of interest are production and annihilation since they both change the total amount of dark matter. The Feynman diagrams for the production processes are shown in figure 4.1, where the annihilation processes are simply their time reversal. The processes which produce dark matter are as follows: the decay of the $Z^{\prime}$, s-channel production from Standard Model fermion annihilation, and t and u-channel production from $Z^{\prime}$ annihilation. The amplitudes and cross sections for these processes are given in Appendix A. There is also the $\chi \chi \rightarrow \chi \chi \chi \chi$ process, where the 


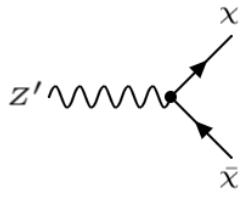

(a)

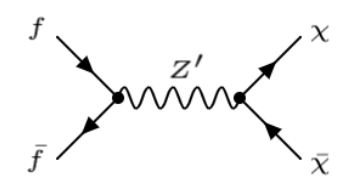

(b)

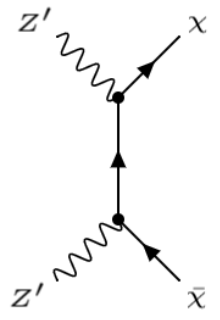

(c)

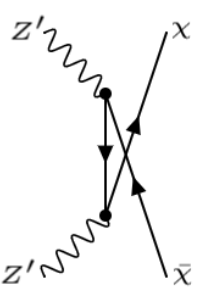

(d)

Figure 4.1: The Feynman diagrams for the dark matter production processes in our model. Subfigure (a) is the $Z^{\prime}$ decay into dark matter, subfigure (b) is the s-channel Standard Model fermion annihilation into dark matter through the $Z^{\prime}$, finally (c) and (d) are the $\mathrm{t}$ and $\mathrm{u}$-channels respectively for the $Z^{\prime}$ annihilation into dark matter through the dark matter fermion.

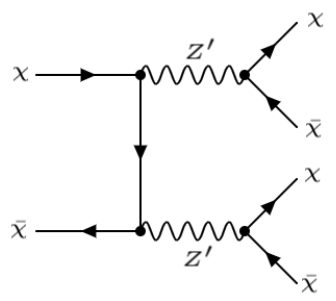

Figure 4.2: Feynman diagram for the $\chi \chi \leftrightarrow \chi \chi \chi \chi$ process which could lead to a dark sector freeze-out (not considered in this work).
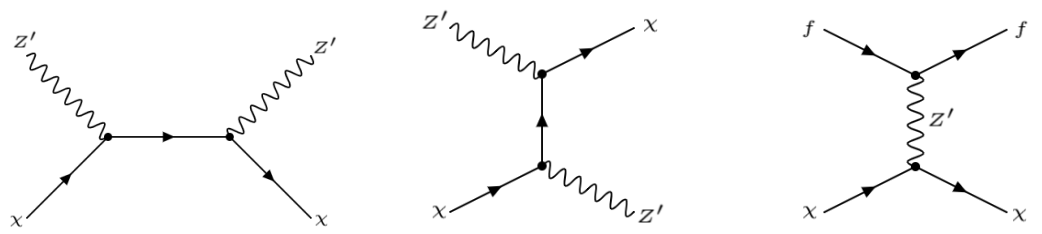

Figure 4.3: Feynman diagrams for scattering processes which may lead to kinetic equilibrium between $\chi, Z^{\prime}$, and the Standard Model sector. 
Feynman diagram is drawn in Figure 4.2, although it is heavily suppressed by dark couplings to the eighth power, therefore we do not consider it. In this model, there also exist diagrams that do not change the number density of dark matter but may lead to kinetic equilibrium between species, such as scatterings $\chi Z^{\prime} \rightarrow \chi Z^{\prime}$ (s-channel and t-channel exchange of $\chi$ ) and $\chi f \rightarrow \chi f$ (t-channel exchange of $Z^{\prime}$ ) which are shown in Figure 4.3.

Chapter 3 goes over in detail how the Boltzmann equation is used to calculate the yield of dark matter as a function of temperature, the so called evolution of the dark matter density. Inputting the interaction rate densities for the processes of our model, the dark matter yield is dictated by,

$$
\frac{d Y_{\chi}}{d x}=\frac{\tilde{g}_{*}}{H s x}\left(R_{Z^{\prime} \leftrightarrow \chi \bar{\chi}}^{\chi}(x)+R_{Z^{\prime} Z^{\prime} \leftrightarrow \chi \bar{\chi}}^{\chi}(x)+R_{f \bar{f} \leftrightarrow \chi \bar{\chi}}^{\chi}(x)\right)
$$

where

$$
R_{Z^{\prime} \leftrightarrow \chi \chi}^{\chi}(x)=R_{Z^{\prime} \rightarrow \chi \bar{\chi}}^{\chi}(x)-R_{\chi \bar{\chi} \rightarrow Z^{\prime}}\left(x, Y_{\chi}\right)=\left(n_{Z^{\prime}}-n_{Z^{\prime}} \frac{n_{\chi}^{2}}{\left(n_{\chi}^{e q}\right)^{2}}\right) \Gamma_{Z^{\prime} \rightarrow \chi \chi}
$$

is the interaction rate density for $Z^{\prime} \leftrightarrow \chi \bar{\chi}$, which is calculated using equations 3.62 and 3.63. The $Z^{\prime}$ is assumed to be part of the thermal bath ${ }^{11}$ until $T<m_{Z^{\prime}}$, when the abundance becomes Boltzmann suppressed since it is too massive to be produced. The decay rate is given by,

$$
\Gamma_{Z^{\prime} \rightarrow \chi \chi / f f}=\frac{m_{Z^{\prime}} \sqrt{1-\frac{4 m_{\chi / f}^{2}}{m_{Z^{\prime}}^{2}}}}{12 \pi}\left[V_{\chi / f}^{2}\left(1+\frac{2 m_{\chi / f}^{2}}{m_{Z^{\prime}}^{2}}\right)+A_{\chi / f}^{2}\left(1-\frac{4 m_{\chi / f}^{2}}{m_{Z^{\prime}}^{2}}\right)\right]
$$

\footnotetext{
${ }^{11}$ This is due to the couplings between $Z^{\prime}$ and the Standard Model fermions being the same as the $Z$ boson, which the couplings are sufficiently high to thermalize.
} 
and from equation 3.60 the interaction rate density for $i i \leftrightarrow \chi \bar{\chi}$ is,

$$
R_{i \bar{i} \leftrightarrow \chi \bar{\chi}}^{\chi}(x)=R_{i \bar{i} \rightarrow \chi \bar{\chi}}^{\chi}(x)-R_{\chi \bar{\chi} \rightarrow i \bar{i}}^{\chi}\left(x, Y_{\chi}\right)=n_{i}^{2}<\sigma v>_{i \bar{i} \rightarrow \chi \bar{\chi}}-n_{\chi}^{2}<\sigma v>_{\chi \bar{\chi} \rightarrow i \bar{i}}
$$

where in our case $i$ is either $Z^{\prime}$ or $f$.

In order to speed up computation time for computing the dark matter abundance, we use approximate expressions for the interaction rates where possible. These interaction rates are inputted into my Python code where the dark matter abundance is computed as a function of $x$ for the given parameters in the appropriate production mechanism regime. When computing the abundance for the freeze-in mechanism, only the first term in equation 4.8 is used, $R_{i \bar{i} \rightarrow \chi \bar{\chi}}^{\chi}$ which can be written as,

$$
R_{i \bar{i} \rightarrow \chi \bar{\chi}}^{\chi}=\frac{\gamma_{i}^{2} T S_{f}}{32(2 \pi)^{6}} \int d s_{m} \sqrt{s_{m}-4 m_{\chi}^{2}} \sqrt{s_{m}-4 m_{i}^{2}} \frac{1}{\sqrt{s_{m}}} K_{1}\left(\frac{\sqrt{s_{m}}}{T}\right) \int|\bar{M}|^{2} d \Omega
$$

where $K_{i}$ is the modified Bessel function of the second kind of order $i$. I go over the approximations for the production process calculations in the following subsections, however the annihilation calculations follow similarly. No approximation is necessary for $Z^{\prime} \leftrightarrow \chi \bar{\chi}$ since the rate density does not involve an integral over $s_{m}$.

\subsection{1 $Z^{\prime} Z^{\prime} \leftrightarrow \chi \bar{\chi}$}

For the $\mathrm{t}$ and $\mathrm{u}$ channel process, $Z^{\prime} Z^{\prime} \leftrightarrow \chi \chi$, the squared amplitude integrated over $\theta$ is simplified using the approximation $\sqrt{s_{m}} \gg m_{Z^{\prime}}$. Dropping terms proportional to 
$m_{Z^{\prime}}$ we arrive at,

$$
\begin{aligned}
\int|\bar{M}|^{2} d \Omega & \approx \frac{64 \pi}{m_{Z^{\prime}}^{4}}\left[\frac { 1 } { m _ { Z ^ { \prime } } ^ { 4 } + m _ { \chi } ^ { 2 } s _ { m } } \left(A_{\chi}^{4}\left(2 m_{\chi}^{4} s_{m}^{2}-2 m_{Z^{\prime}}^{8}+m_{\chi}^{2} m_{Z^{\prime}}^{4} s_{m}\right)\right.\right. \\
& \left.+2 A_{\chi}^{2} m_{Z^{\prime}}^{4} V_{\chi}^{2}\left(-7 m_{\chi}^{2} s_{m}-6 m_{Z^{\prime}}^{4}\right)-m_{Z^{\prime}}^{4} V_{\chi}^{4}\left(m_{\chi}^{2} s_{m}+2 m_{Z^{\prime}}^{4}\right)\right) \\
& -\left(A_{\chi}^{4}\left(-4 m_{\chi}^{4}+m_{Z^{\prime}}^{4}+4 m_{\chi}^{2} m_{Z^{\prime}}^{2}\right)+2 A_{\chi}^{2} m_{Z^{\prime}}^{2} V_{\chi}^{2}\left(2 m_{\chi}^{2}+3 m_{Z^{\prime}}^{2}\right)\right. \\
& \left.\left.+m_{Z^{\prime}}^{4} V_{\chi}^{4}\right)\left(-\log \left(\frac{s_{m}}{\left(m_{\chi}\right)^{2}}\right)\right)\right]
\end{aligned}
$$

where the full expression for the squared amplitude, $|\bar{M}|^{2}$, is given in Appendix A. This simplifies the expression quite nicely and works well for all temperatures since when $T \sim \sqrt{s_{m}} \leq m_{Z^{\prime}}$ the rate is Boltzmann suppressed and very small. There does not exist a useful approximation of the rate itself, so the rate is computed by numerically integrating the approximation in equation 4.10 over $s_{m}$. However we do notice that for $T \gg m_{Z^{\prime}}, m_{\chi}$ we have $R_{Z^{\prime} Z^{\prime} \leftrightarrow \chi \chi} \propto T^{6}$. Figure 4.4 shows the production rate density for the $Z^{\prime} Z^{\prime} \rightarrow \chi \chi$ process with (dashed blue) and without (solid black) the approximations for a particular set of parameters, showing agreement between the approximate and full (numerical) expressions.

\subsection{2 $f f \leftrightarrow \chi \chi$}

The s-channel process $(f f \leftrightarrow \chi \chi)$ has three main temperature regimes: high temperature where $T \gg m_{Z^{\prime}}$ (light regime), low temperature where $T \ll m_{Z^{\prime}}$ (heavy regime), and the resonant regime where $T \approx m_{Z^{\prime}}$. Figure 4.5 shows the production rate density for the s-channel process with (dashed coloured) and without (solid black) the approximations for a particular set of parameters, showing agreement be-

tween the approximate and full expressions in the appropriate regimes. Note that the Boltzmann suppression of the rate density occurs when $T<m_{\chi}$ since $m_{\chi}>m_{e}$. This 


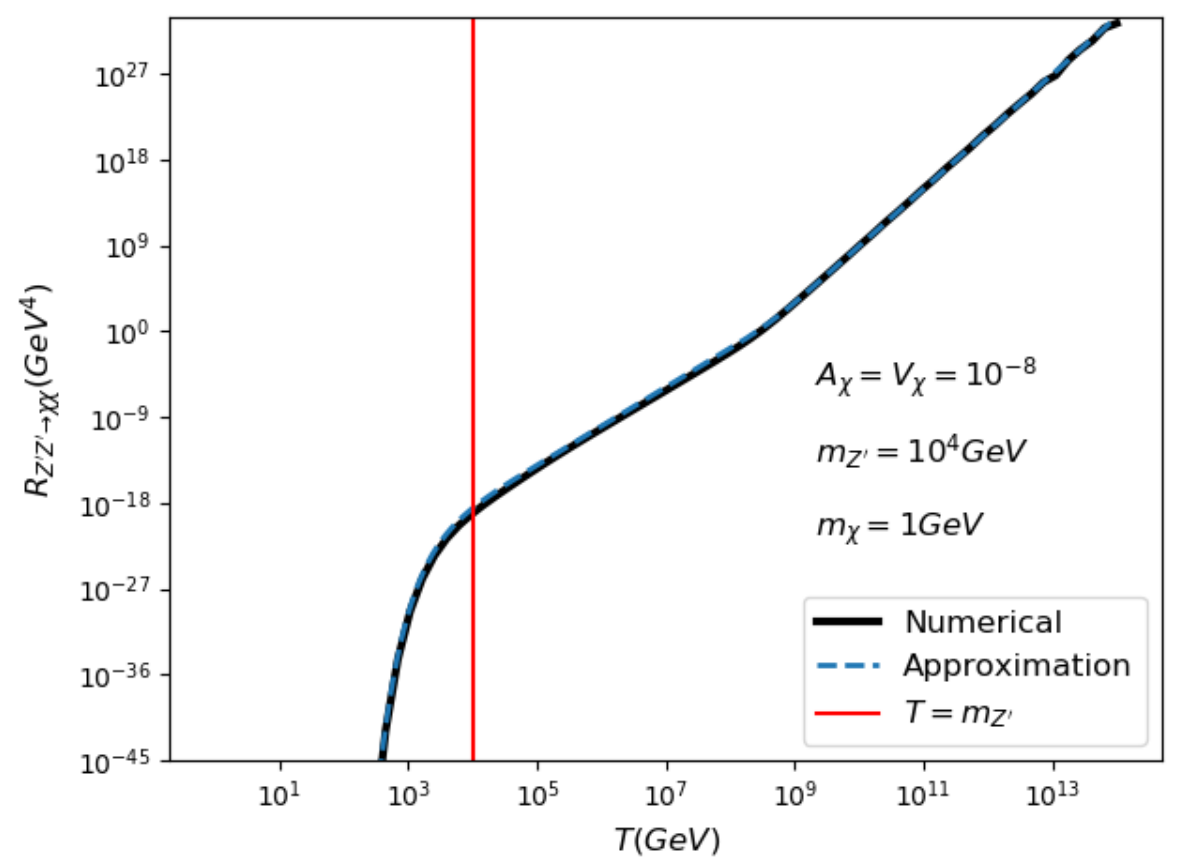

Figure 4.4: The production rate density for $Z^{\prime}$ annihilation (t/u-channel) is plotted as a function of temperature. The full squared amplitude numerically integrated over $s_{m}$ and $\theta$ (solid black) and under the approximation $\sqrt{s_{m}} \sim T \gg m_{Z^{\prime}}$ (dashed blue) are compared. The line corresponding to $T=m_{Z^{\prime}}$ is plotted in red. At high temperatures $\left(T>m_{Z^{\prime}}\right) R \propto T^{6}$, and at low temperatures $\left(T<m_{Z^{\prime}}\right)$ the $Z^{\prime}$ abundance is Boltzmann suppressed thus $R$ drops near zero. 


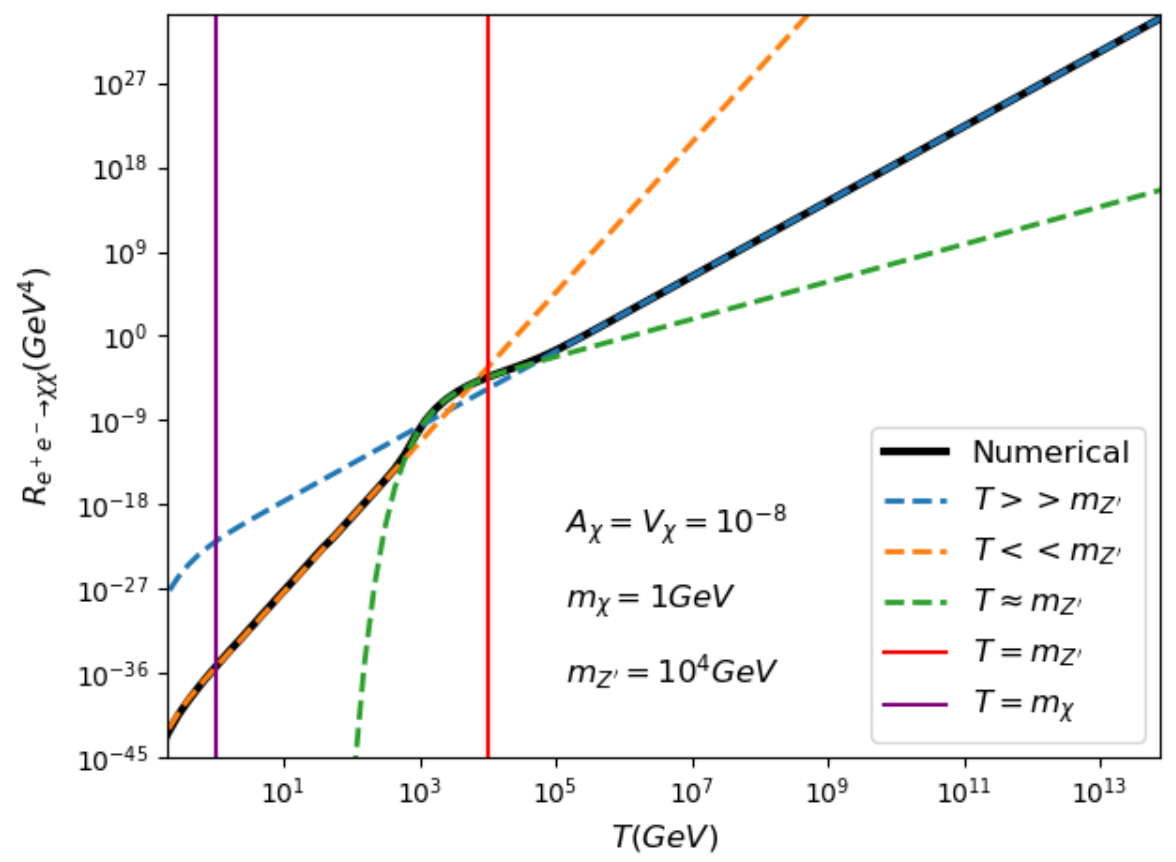

Figure 4.5: The production rate density for electron positron annihilation (schannel) is plotted as a function of temperature. The full squared amplitude numerically integrated over $s_{m}$ (solid black) and under the three approximations of each temperature regime (dashed coloured) are compared. The lines corresponding to $T=m_{Z^{\prime}}$ and $T=m_{\chi}$ are plotted as red and purple vertical lines respectively. For high temperatures $R \propto T^{4}$ and for low temperatures, but for $T>m_{\chi}, R \propto T^{7}$. 
is in contrast to Figure 4.4, where Boltzmann suppression occurs when $T<m_{Z^{\prime}}$ as the temperature is no longer sufficient to produce $Z^{\prime}$ particles. The temperature at which Boltzmann suppression occurs for a given process is whichever happens first: either when the abundance of the initial species become small, or if the final particles are much more massive than the initial, when the temperature drops below the mass of the final particles.

\section{Light Regime $\left(T \gg m_{Z^{\prime}}\right)$}

For the light regime since $T \gg m_{Z^{\prime}}, s_{m} \gg m_{Z^{\prime}}^{2}$ therefore $\frac{m_{Z^{\prime}}^{2}}{s_{m}}$ can be neglected. The rate in this regime is given by,

$$
R_{f f \rightarrow \chi \chi} \approx \frac{\gamma_{f}^{2} T^{2} m_{\chi}^{2} K_{2}\left(\frac{2 m_{\chi}}{T}\right)}{192 \pi^{5}}\left(A_{f}^{2} A_{\chi}^{2}\left(1+\frac{12 m_{f}^{2} m_{\chi}^{2}}{m_{Z^{\prime}}^{4}}\right)+V_{\chi}^{2} A_{f}^{2}+V_{f}^{2} A_{\chi}^{2}+V_{f}^{2} V_{\chi}^{2}\right)
$$

\section{Heavy Regime $\left(T \ll m_{Z^{\prime}}\right)$}

For the heavy regime $T \ll m_{Z^{\prime}}$ and $s_{m} \ll m_{Z^{\prime}}^{2}$ therefore $\frac{s_{m}}{m_{Z^{\prime}}^{2}}$ can be neglected, so the rate becomes,

$$
\begin{aligned}
R_{f f \rightarrow \chi \chi} & \approx \frac{\gamma_{f}^{2} T^{4}}{768 \pi^{5} m_{Z^{\prime}}^{4}}\left(A\left[b^{2} K_{0}(b)+2 b K_{1}(b)\right]\right. \\
& +B T^{2}\left[K_{0}(b)\left(b^{4}+8 b^{2}\right)+K_{1}(b)\left(4 b^{3}+16 b\right)\right] \\
& \left.+C T^{4}\left[K_{0}(b)\left(b^{6}+24 b^{4}+192 b^{2}\right)+K_{1}(b)\left(6 b^{5}+96 b^{3}+384 b\right)\right]\right)
\end{aligned}
$$

where,

$$
\begin{gathered}
b \equiv \frac{2 m_{\chi}}{T}, \\
A \equiv m_{f}^{2} m_{\chi}^{2}\left(28 A_{f}^{2} A_{\chi}^{2}-8 A_{f}^{2} V_{\chi}^{2}-8 V_{f} A_{\chi}^{2}+4 V_{f}^{2} V_{\chi}^{2}\right),
\end{gathered}
$$




$$
\begin{aligned}
B & \equiv-24 \frac{A_{f}^{2} A_{\chi}^{2} m_{f}^{2} m_{\chi}^{2}}{m_{Z^{\prime}}^{2}}-4 A_{f}^{2} A_{\chi}^{2}\left(m_{f}^{2}+m_{\chi}^{2}\right)-4 A_{f}^{2} V_{\chi}^{2} m_{f}^{2}+2 A_{f}^{2} V_{\chi}^{2} m_{\chi}^{2}+2 V_{f}^{2} A_{\chi}^{2} m_{f}^{2} \\
& +2 V_{f}^{2} V_{\chi}^{2}\left(m_{f}^{2}+m_{\chi}^{2}\right)-4 V_{f}^{2} A_{\chi}^{2} m_{\chi}^{2}
\end{aligned}
$$

and

$$
C \equiv A_{f}^{2} A_{\chi}^{2}\left(12 \frac{m f^{2} m_{\chi}^{2}}{m_{Z^{\prime}}^{4}}+1\right)+A_{f}^{2} V_{\chi}^{2}+V_{f}^{2} A_{\chi}^{2} V_{f}^{2} V_{\chi}^{2}
$$

Resonance Regime $\left(T \approx m_{Z^{\prime}}\right)$

Near the resonance $T \approx m_{Z^{\prime}}$ we apply the narrow width approximation [112] to obtain an expression for the rate. The interaction rate is given by,

$$
\begin{aligned}
R_{f f \rightarrow \chi \chi} & \approx \sqrt{m_{Z^{\prime}}^{2}-4 m_{\chi}^{2}} \sqrt{m_{Z^{\prime}}^{2}-4 m_{f}^{2}} K_{1}\left(\frac{m_{Z^{\prime}}}{T}\right) \frac{\gamma_{f}^{2} T}{192 \pi^{4} m_{Z^{\prime}}^{2} \Gamma_{Z^{\prime}}}\left\{A _ { f } ^ { 2 } \left[A _ { \chi } ^ { 2 } \left(16 m_{f}^{2} m_{\chi}^{2}\right.\right.\right. \\
& \left.+m_{Z^{\prime}}^{4}-4 m_{Z^{\prime}}^{2}\left(m_{f}^{2}+m_{\chi}^{2}\right)-V_{\chi}^{2}\left(8 m_{f}^{2} m_{\chi}^{2}+4 m_{Z^{\prime}}^{2} m_{f}^{2}-2 m_{Z^{\prime}}^{2} m_{\chi}^{2}-m_{Z^{\prime}}^{4}\right)\right] \\
& +V_{f}^{2}\left[2 A_{\chi}^{2} m_{f}^{2} m_{Z^{\prime}}^{2}-8 A_{\chi}^{2} m_{f}^{2} m_{\chi}^{2}+4 V_{\chi}^{2} m_{f}^{2} m_{\chi}^{2}+2 V_{\chi}^{2} m_{Z^{\prime}}^{2} m_{f}^{2}+A_{\chi}^{2} m_{Z^{\prime}}^{2}\right. \\
& \left.\left.-4 A_{\chi}^{2} m_{\chi}^{2} m_{Z^{\prime}}^{2}+2 V_{\chi}^{2} m_{\chi}^{2} m_{Z^{\prime}}^{2}+V_{\chi}^{2} m_{Z^{\prime}}^{2}\right]\right\}
\end{aligned}
$$

where $\Gamma_{Z^{\prime}}$ is the total width of $Z^{\prime}$ given by,

$$
\Gamma_{Z^{\prime}}=\Gamma_{Z^{\prime} \rightarrow \chi \chi}+\sum_{i} \Gamma_{Z^{\prime} \rightarrow f_{i} f_{i}}
$$

\subsection{UV Completion}

Our model is far from perfect, especially at large masses and energies. As discussed in Ref. [113], simple dark matter models often violate perturbative unitarity and gauge invariance. This is due to the fact that the amplitudes increase infinitely with the center of mass energy. We can compute an upper bound on the energy such that 
unitarity is not violated, as is calculated in Ref. [104], which acts not as a physical constraint but an upper bound on the validity of our amplitude calculations. For energies above this bound, a more complete field theory is required to accurately calculate the amplitudes [114]. 


\section{Chapter 5}

\section{Results}

Given the processes of our model as discussed in Chapter 4, the evolution of the dark matter yield and the final relic abundance is calculated as a function of the free parameters of our model namely $m_{Z^{\prime}}, m_{\chi}, A_{\chi}$, and $V_{\chi}$. The parameters which give rise to the observed relic abundance, $\Omega h^{2}=0.12$ [20], are found for both production mechanisms of interest: freeze-in and freeze-out. The reheating temperature, $T_{R}$, is assumed to be $10^{14} \mathrm{GeV}$ throughout this thesis which is sufficiently larger than the lower bound found in Ref. [115].

\subsection{Determining the Production Regime}

We are interested in how the free parameters of our model influence the way in which dark matter is produced in the early universe. Chapter 2 explains a few of the dark matter production mechanisms in the literature, although for this study we only focus on freeze-out and freeze-in. For a given set of parameters, in order to determine which mechanism is responsible for dark matter production, we must compare the time scale of the universe's expansion to the frequencies of production interactions, $W$, which 
are given in equations 3.66 and 3.67, $W=n<\sigma v>_{12 \rightarrow 34}$ for $12 \rightarrow 34$ processes and $W=\Gamma_{1 \rightarrow 23}$ for $1 \rightarrow 23$ processes. If the frequency of production exceeds the Hubble rate, dark matter thermalizes with the bath and eventually decouples and freezes-out. Thermalization occurs if chemical and kinetic equilibrium are reached. If enough dark matter is produced, kinetic equilibrium can occur from scattering between species as in Figure 4.3. Chemical equilibrium happens when enough dark matter exists, so production and annihilation processes occur at the same rate. If thermalization never occurs dark matter is produced through the freeze-in mechanism. For each set of values, $m_{Z^{\prime}}, m_{\chi}$, and dark matter couplings $\left(A_{\chi}, V_{\chi}\right)$, the production mechanism is determined in order to map out the mechanisms on the parameter space. The frequency of production is compared to the Hubble rate (equation 3.10) for each process at all temperatures from reheating until Boltzmann suppression (when the interaction rates exponentially fall to zero). The thermalization condition is,

$$
\exists T: T_{B}<T<T_{R} \wedge W_{12 / 1 \rightarrow \chi \bar{\chi}}(T)>H(T)
$$

where $T_{R}$ is the reheating temperature and $T_{B}$ is the Boltzmann suppression temperature. This condition is checked for each process, namely each s-channel process involving Standard Model leptons, neutrinos, and quarks, the $Z^{\prime}$ decay, and annihilation of two $Z^{\prime}$ particles. 

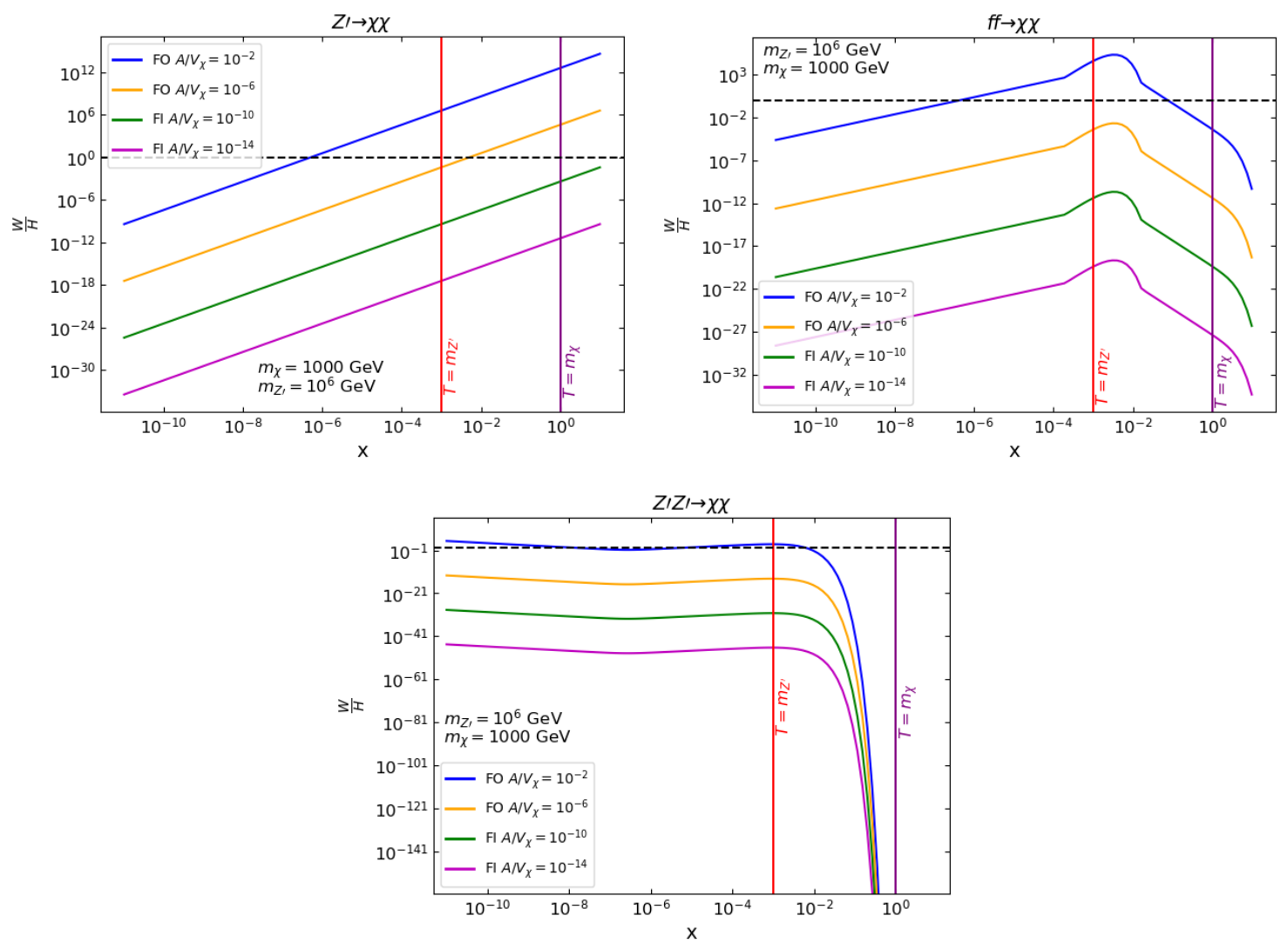

Figure 5.1: The ratio of the production frequency labeled $W$ to the Hubble parameter labeled $H$ as a function of $\mathrm{x}$ for various dark matter couplings. All three production channels are shown. The dashed black lines show where the ratio is equal to 1 (where $W=H$ ). If at least one of the processes is above the dashed line we assume that chemical equilibrium is reached and dark matter is produced through freeze-out (FO). If no processes have a large enough $W$ then we assume dark matter is produced through freeze-in (FI). A specific example is shown where $m_{\chi}=1000 \mathrm{GeV}$ and $m_{Z^{\prime}}=10^{6} \mathrm{GeV}$.

Figure 5.1 shows three subplots of the ratio of the frequency of interactions to the Hubble rate as a function of $x=\frac{m_{\chi}}{T}$ for each production mechanism. For efficiency, each process is checked at temperatures where $\frac{W}{H}$ is maximum, since if the maximum is not greater than 1 then the Hubble rate is certainly greater than $W$ for all temperatures. The s-channel processes are only checked at temperatures near the $Z^{\prime}$ mass since the rates are maximum at $T \approx m_{Z^{\prime}}$ due to the resonance. The $Z^{\prime} Z^{\prime} \rightarrow \chi \chi$ 
process is checked at the reheating temperature, $T_{R}$, since the rates increase rapidly with temperature and are largest at reheating and temperatures above. The ratio of the $Z^{\prime}$ decay frequency to the Hubble rate follows an inverse power law with temperature, so this ratio is largest at low temperatures. Hence for efficiency, the ratio is checked for the lowest temperatures before the $Z^{\prime}$ becomes Boltzmann suppressed, near the mass of $Z^{\prime}$. $W_{Z^{\prime} \rightarrow \chi \chi}$ alone does not give any information on the number density of the $Z^{\prime}$, thus $W$ could still be large while there are virtually no more $Z^{\prime}$ particles in the bath. The interaction rate $R_{Z^{\prime} \rightarrow \chi \chi}=\Gamma_{Z^{\prime} \rightarrow \chi \chi} n_{Z^{\prime}}$ is checked alongside

the ratio in order to ensure that if $\frac{W}{H}>1$ the rate is not negligible. If $R_{Z^{\prime} \rightarrow \chi \chi} \approx 0, Z^{\prime}$ particles are Boltzmann suppressed therefore the thermalization condition cannot be met since there would not be enough $Z^{\prime}$ particles to sufficiently produce dark matter. These methods are used in producing the phase diagram plots in section 5.3.

\subsection{Dark Matter Evolution}

We now have all of the necessary tools to study the evolution of dark matter in our model. The evolution of dark matter is plotted as a function of $x$ in Figure 5.2, showing the evolution of its yield through time. The Figure has four subfigures for two $Z^{\prime}$ masses and two dark matter masses, each showing the evolution with varying dark matter couplings. As $A_{\chi}$ and $V_{\chi}$ increase the final yield increases up until the freeze-out regime is reached and then the final yield begins to decrease. This is due to the fact that while the dark matter couplings are small we are in the freeze-in regime and since production dominates over annihilation, larger couplings result in more production thus more dark matter. At larger couplings where equilibrium is reached we now have annihilation processes dominating the yield just before freezeout. Therefore larger couplings result in more annihilations so the equilibrium curve 

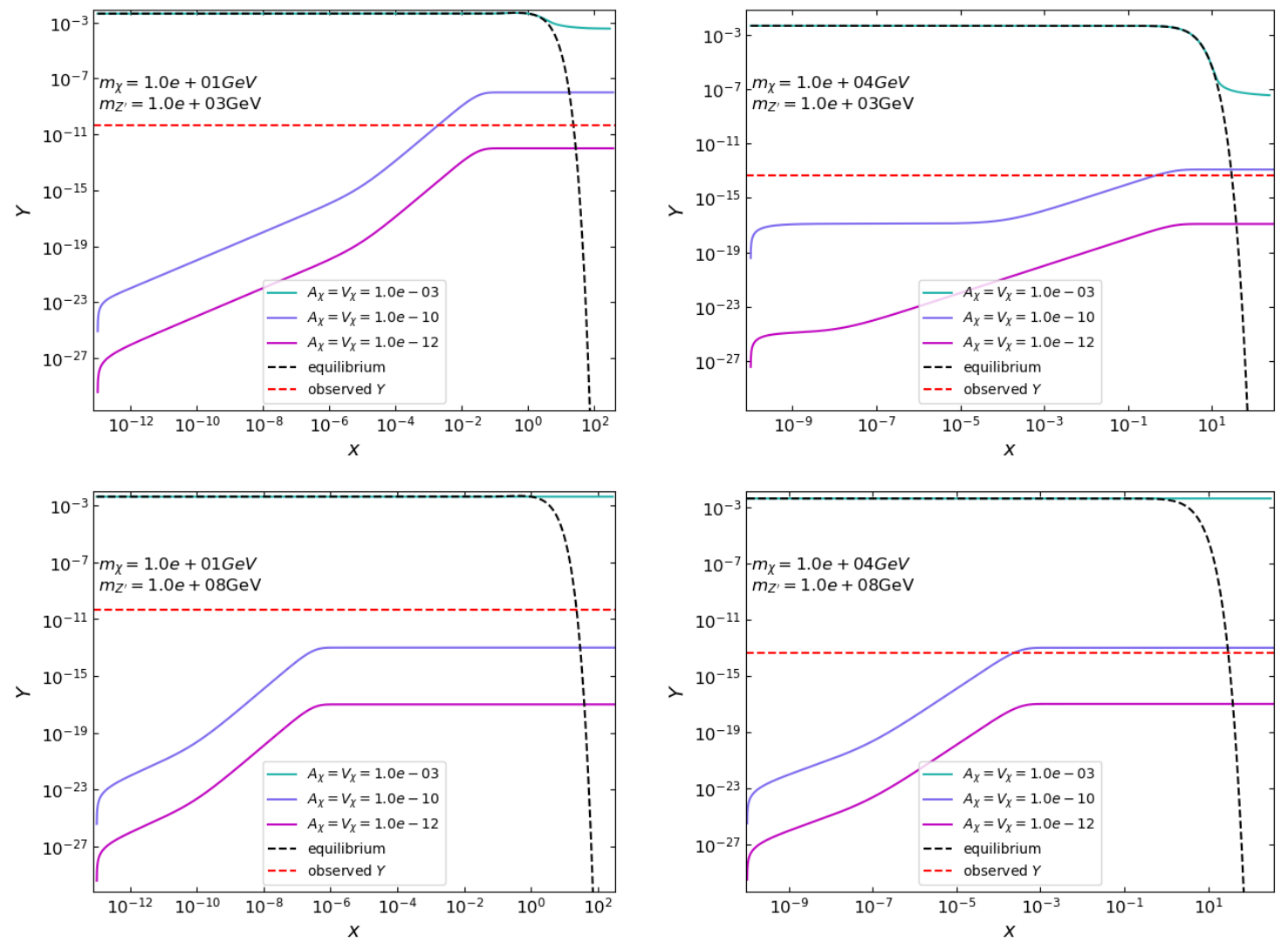

Figure 5.2: The evolution of the yield of dark matter for different couplings $\left(A_{\chi}, V_{\chi}\right)$. The horizontal axis is $x=\frac{m_{\chi}}{T}$ which flows in the direction of time. The red dashed line shows the yield which gives the observed relic abundance, $\Omega h^{2}=0.12$ [20]. The top left plot has $m_{Z^{\prime}}=10^{3} \mathrm{GeV}$ and $m_{\chi}=10 \mathrm{GeV}$. The top right has $m_{Z^{\prime}}=10^{3} \mathrm{GeV}$ and $m_{\chi}=10^{4} \mathrm{GeV}$. The bottom left has $m_{Z^{\prime}}=10^{8} \mathrm{GeV}$ and $m_{\chi}=10 \mathrm{GeV}$. The bottom right has $m_{Z^{\prime}}=10^{8} \mathrm{GeV}$ and $m_{\chi}=10^{4} \mathrm{GeV}$. 

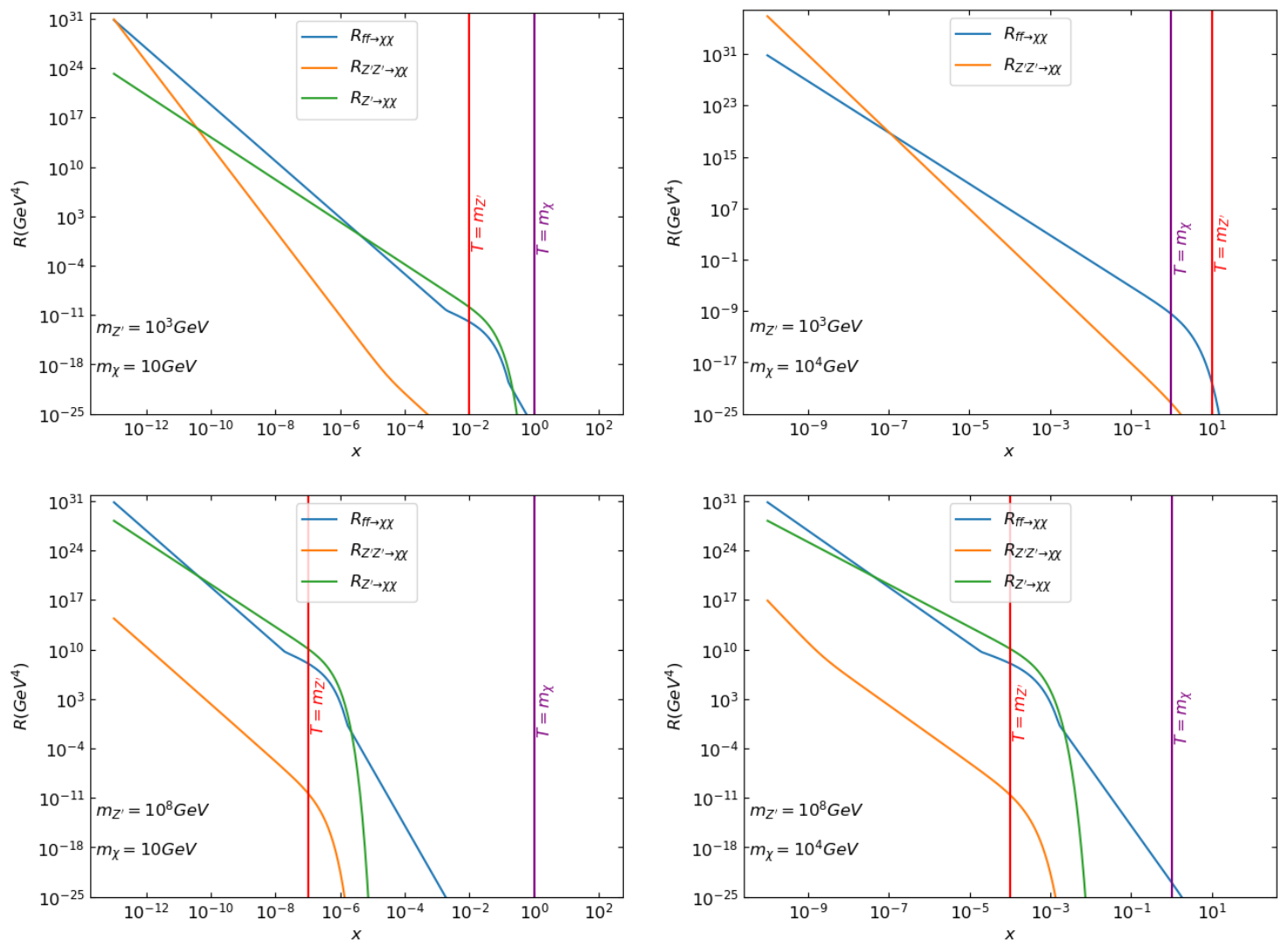

Figure 5.3: The interaction rate densities, $R$, for all three production processes at different $Z^{\prime}$ and $\chi$ masses as a function of $x=\frac{m_{\chi}}{T} . A_{\chi}=V_{\chi}=10^{-10}$, corresponding to the freeze-in regime, for all four subfigures. $R_{f f \rightarrow \chi \chi}$ is plotted in blue, $R_{Z^{\prime} Z^{\prime} \rightarrow \chi \chi}$ is in orange, and $R_{Z^{\prime} \rightarrow \chi \chi}$ in green. The vertical lines correspond to $T=m_{Z^{\prime}}$ (red) and $T=m_{\chi}$ (purple).

is tracked for longer, resulting in a lower final yield. If the interaction rates are large enough for thermalization, but low enough at temperatures greater than $m_{\chi}$ we see ultra-relativistic freeze-out. This ultra-relativistic freeze-out can be seen in the lower subfigures of Figure 5.2 as shown in light green. In order to understand the effect each process has on the evolution, we must compare the interaction rate densities as plotted in Figures 5.3 and 5.4. The rates are overall more suppressed when the $Z^{\prime}$ mass is larger for all processes. The process $R_{Z^{\prime} Z^{\prime} \leftrightarrow \chi \chi}$ is proportional to $\frac{1}{m_{Z^{\prime}}^{4}}$, while the process $Z^{\prime} \leftrightarrow \chi \chi$ is suppressed at $T \approx m_{Z^{\prime}}$ hence for large $Z^{\prime}$ masses $R_{Z^{\prime} \leftrightarrow \chi \chi}$ drops 

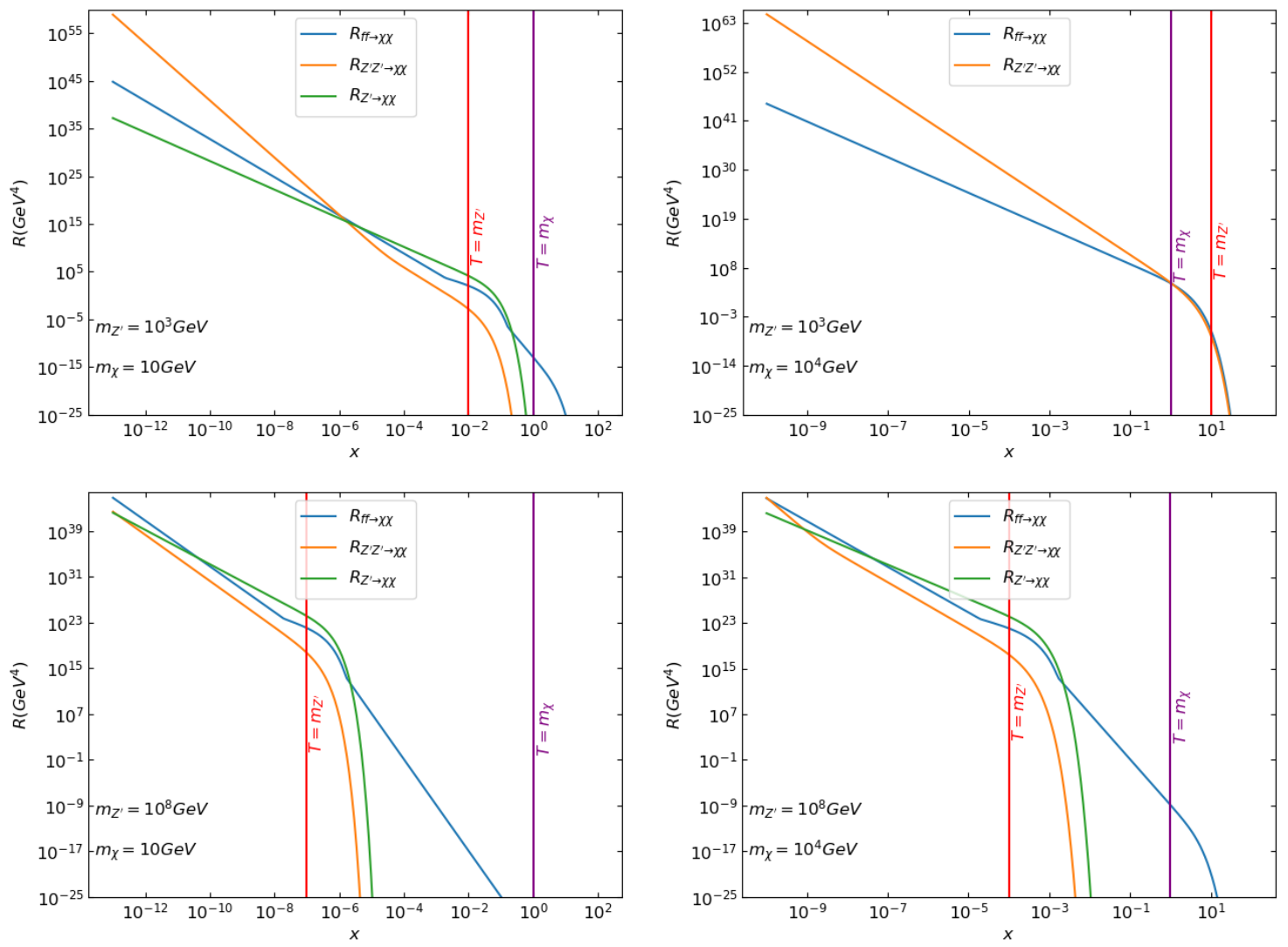

Figure 5.4: The interaction rate densities, $R$, for all three production processes at different $Z^{\prime}$ and $\chi$ masses as a function of $x=\frac{m_{\chi}}{T}$. $A_{\chi}=V_{\chi}=10^{-3}$ for all four subfigures, corresponding to the freeze-out regime. $R_{f f \rightarrow \chi \chi}$ is plotted in blue, $R_{Z^{\prime} Z^{\prime} \rightarrow \chi \chi}$ is in orange, and $R_{Z^{\prime} \rightarrow \chi \chi}$ in green. The vertical lines correspond to $T=m_{Z^{\prime}}$ (red) and $T=m_{\chi}$ (purple). 
to zero earlier (at a higher temperature). In addition, the low temperature regime $\left(T \ll m_{Z^{\prime}}\right)$ of $R_{f f \rightarrow \chi \chi}$ has a larger slope than the high temperature regime. Therefore for a larger $m_{Z^{\prime}}$ the low temperature regime occurs at a higher temperature and the large slope drops the rate near zero faster. If $2 m_{\chi}>m_{Z^{\prime}}$ the $Z^{\prime}$ decay is kinematically not allowed so this leaves $Z^{\prime} Z^{\prime} \rightarrow \chi \chi$ and $f f \rightarrow \chi \chi$ for dark matter production. This is the case for the freeze-in curves in the top right subfigure of Figure 5.2, where $Z^{\prime} Z^{\prime} \rightarrow \chi \chi$ dominates early on while $f f \rightarrow \chi \chi$ begins to dominate later where the slope increases. The cross over between each process dominating is evident in the top right subfigure of Figure 5.3. Dark matter freezes-in (the yield flat-lines) at $T \approx m_{\chi}$ since it is kinematically unfavourable to produce dark matter when $T<m_{\chi}$. For the freeze-in curves in the other three subfigures of Figure 5.2, $2 m_{\chi}<m_{Z^{\prime}}$ therefore the decay will occur. The decay process will dominate dark matter production at freeze-in and early on the s-channel dominates. $Z^{\prime} Z^{\prime} \rightarrow \chi \chi$ is more important if $m_{Z^{\prime}}$ is small, although as illustrated in Figure 5.3 for large $Z^{\prime}$ masses this process is negligible. The cross over between the s-channel and decay dominating is shown in the top left and bottom subfigures of Figure 5.3. Dark matter freezes-in when $T \approx m_{Z^{\prime}}$ since at that point $n_{Z^{\prime}}$ becomes small due to the $Z^{\prime}$ Boltzmann suppression which occurs when $T \approx m_{Z^{\prime}}$. The dark matter mass does not have a significant effect on the rates or evolution except for whether $2 m_{\chi}<m_{Z^{\prime}}$ and the decay is allowed, and the ratio $\frac{m_{\chi}}{m_{Z^{\prime}}}$ can affect when $\chi$ decouples. In the freeze-out regime for larger dark matter couplings, since $R_{Z^{\prime} Z^{\prime} \leftrightarrow \chi \chi} \propto \lambda^{4}$ where $\lambda$ is $A_{\chi}$ or $V_{\chi}, Z^{\prime} Z^{\prime} \leftrightarrow \chi \chi$ dominates if $m_{Z^{\prime}}$ is small. For the relativistic freeze-out curves, the lower subfigures of Figure 5.4 show that the rates of all three processes are comparable but low enough such that dark matter freezes-out early on. 


\subsection{Relic Abundance}

We will now explore the parameter space, its effect on the calculated relic abundance, and what parameters give rise to the observed relic abundance. The relic abundance is calculated by the relation,

$$
\Omega_{\chi} h^{2} \equiv \frac{\rho_{\chi}}{\rho_{c}}=\frac{Y_{\infty} m_{\chi} s_{\infty}}{\rho_{c}}
$$

where $\rho_{c} \equiv \frac{3 H_{0}^{2}}{8 \pi G}=1.05 \times 10^{-5} \mathrm{GeV}^{2} \mathrm{~cm}^{-3}$ is the critical energy density which is the energy density required for a spatially flat universe, and $s_{\infty}=2891.2 \mathrm{~cm}^{-3}$ is the present entropy density of the universe [116]. $Y_{\infty}$ is the current dark matter yield, which is calculated by the methods described in Chapter 3.

We calculate the dark matter couplings as a function of the other free parameters at the correct relic abundance under the freeze-in or freeze-out approximation. We will show expressions for the couplings in the freeze-in regime only, since calculating the abundance for freeze-out requires knowing the decoupling temperature. We will fix $A_{\chi}=V_{\chi}=\lambda$ for simplicity to define one variable for the coupling. We can consider the processes separately in order to calculate the coupling required to produce the observed abundance. Just considering the $Z^{\prime} \rightarrow \chi \chi$ process, the required coupling is given by,

$$
\lambda=\sqrt{\frac{\Omega_{\chi} h^{2} 16 \sqrt{\pi} \pi^{6} \rho_{c} m_{\chi}^{2} g_{* s}\left(T_{R}\right)^{2}}{45 \sqrt{5} s_{\infty} \gamma_{Z^{\prime}} m_{Z^{\prime}}^{2} m_{P l} x_{R}^{3} g_{*}\left(T_{R}\right)}\left[K_{3}\left(\frac{x_{R} m_{Z^{\prime}}}{m_{\chi}}\right) \sqrt{1-\frac{4 m_{\chi}^{2}}{m_{Z^{\prime}}^{2}}}\left(1-\frac{m_{\chi}^{2}}{m_{Z^{\prime}}^{2}}\right)\right]^{-1}} .
$$

For the $12 \rightarrow 34$ processes the expression is numerically integrated in order to calcu- 
late the couplings. For the case of $f f \rightarrow \chi \chi$ we have,

$$
\lambda=\sqrt{\frac{4 \pi^{3}}{135} \sqrt{\frac{\pi}{5}} \frac{\Omega_{\chi} h^{2} \rho_{c} m_{\chi}^{4} g_{* s}\left(T_{R}\right)^{2}}{s_{\infty} m_{P l} g_{*}\left(T_{R}\right) \int_{x_{R}}^{\infty} x^{4} \frac{<\sigma v>_{f f \rightarrow \chi \chi}}{\lambda^{2}}\left(n_{f}^{e q}\right)^{2} d x}}
$$

The thermally averaged cross section for $Z^{\prime} Z^{\prime} \rightarrow \chi \chi$ is proportional to $\lambda^{4}$ so we have,

$$
\lambda=\left(\frac{4 \pi^{3}}{135} \sqrt{\frac{\pi}{5}} \frac{\Omega_{\chi} h^{2} \rho_{c} m_{\chi}^{4} g_{* s}\left(T_{R}\right)^{2}}{s_{\infty} m_{P l} g_{*}\left(T_{R}\right) \int_{x_{R}}^{\infty} x^{4} \frac{<\sigma v\rangle_{Z^{\prime} Z^{\prime} \rightarrow \chi \chi}}{\lambda^{4}}\left(n_{Z^{\prime}}^{e q}\right)^{2} d x}\right)^{\frac{1}{4}}
$$

where $\Omega_{\chi} h^{2}=0.1200 \pm 0.0012$ [20]. The values of the effective number of degrees of freedom $g_{*}$ can be taken at $T=T_{R}$, the reheating temperature, if freeze-in occurs before $g_{*}$ begins to decrease (when the top quarks decouple from the bath at $T \approx 170$ $\mathrm{GeV}$ ). While considering all of the processes together, a quadratic equation must be solved since the $\mathrm{t} / \mathrm{u}$-channel cross section is proportional to $\lambda^{4}$ while the decay and s-channel are proportional to $\lambda^{2}$. For the freeze-out regime, the couplings required are calculated using the methods from section 3.3.3.

Given all of the dark matter production and annihilation processes, the relic abundance is calculated as a function of dark matter coupling, mass, and $m_{Z^{\prime}}$. The production mechanism is determined for each parameter. The contour plot in Figure 5.5 shows contours of the relic abundance as a function of dark matter couplings and $Z^{\prime}$ mass, and where the correct relic abundance lies within the parameter space. Both production mechanisms are capable of producing the observed abundance, shown as red contour lines, with the freeze-out curve at large couplings and freeze-in at small couplings. The final relic density is inversely proportional to $m_{Z^{\prime}}$ in the freeze-in regime since $R_{Z^{\prime} Z^{\prime} \rightarrow \chi \chi}$ and $R_{f f \rightarrow \chi \chi}$ decrease while $m_{Z^{\prime}}$ increases at low temperatures. At higher temperatures $R_{Z^{\prime} \rightarrow \chi \chi}$ is proportional to the $Z^{\prime}$ mass, although as the temperature decreases Boltzmann suppression kicks in and the rate drops. From equation 


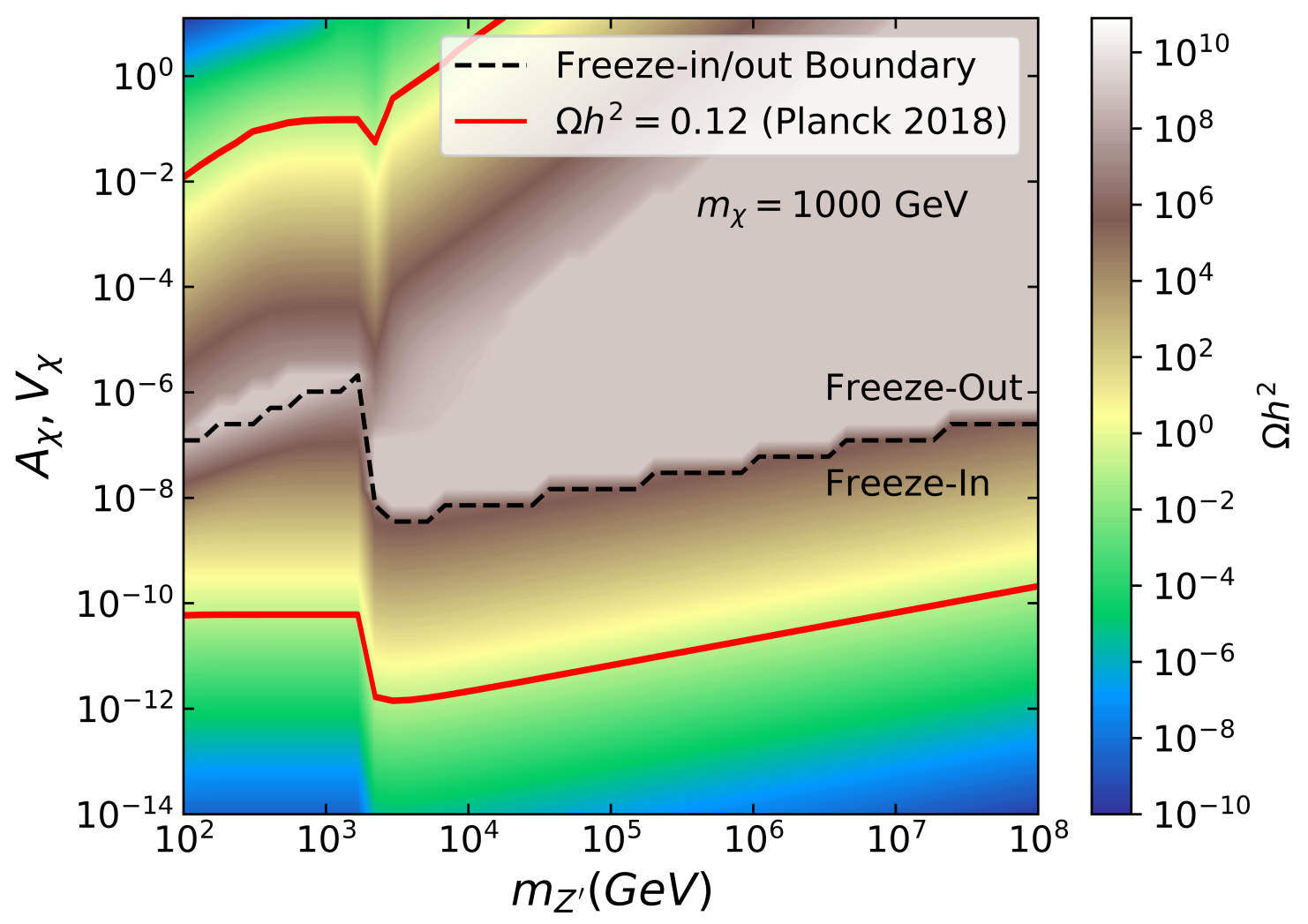

Figure 5.5: Contour plot showing the relic abundance as a function of dark matter couplings and $m_{Z^{\prime}}$ for $m_{\chi}=1000 \mathrm{GeV}$. The dashed line is the freeze-in/out boundary which is determined by comparing the frequency of interactions $W$ to Hubble $H$ for each process and each temperature from reheating until Boltzmann suppression. All values below this boundary are classified as freeze-in and above as freeze-out. The observed relic density is plotted in red for both freeze-out and freeze-in regimes [20]. Notice that the relic abundance in the freeze-out regime decreases as couplings increase, and that the freeze-in relic abundance increases with couplings. 


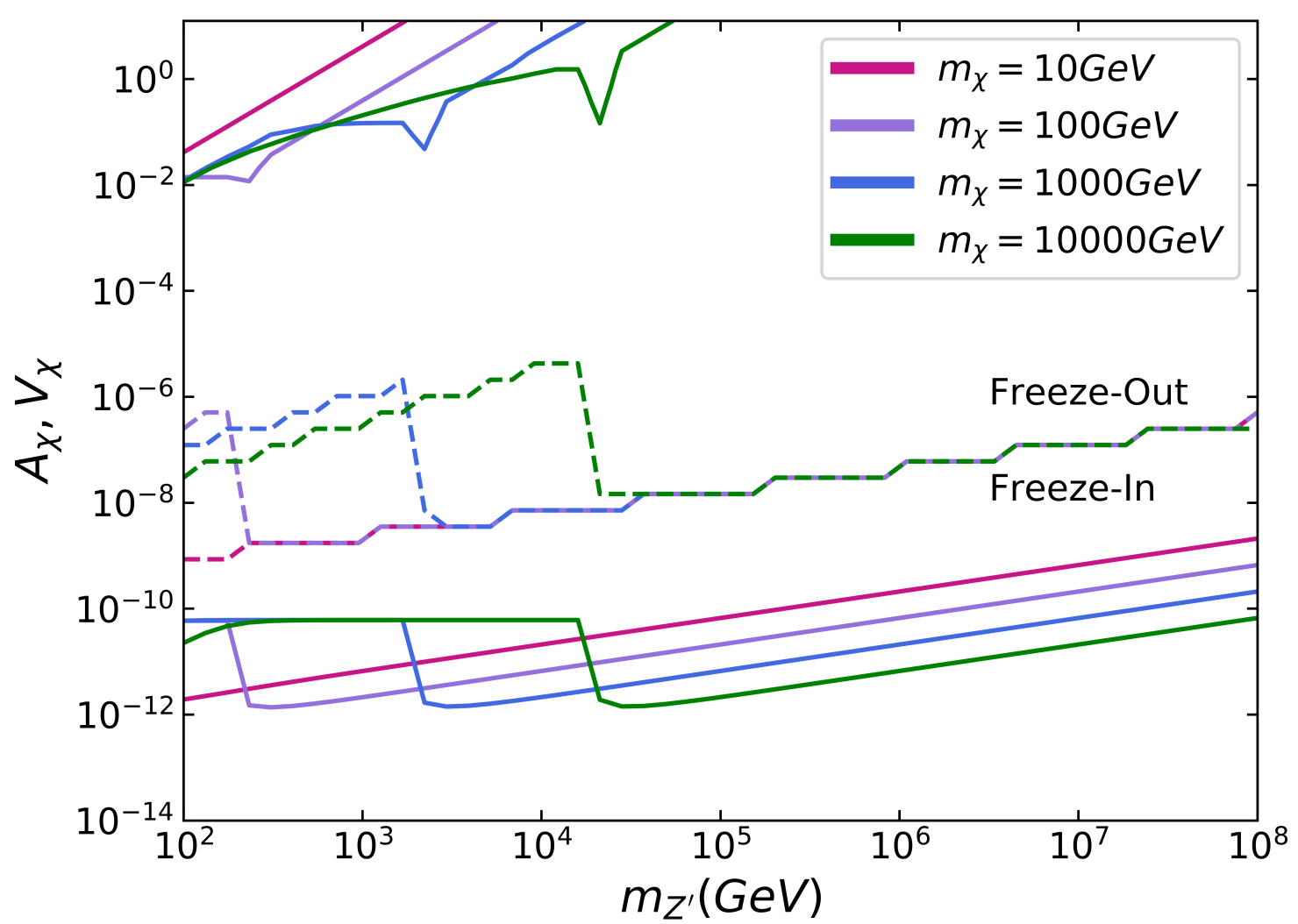

Figure 5.6: Contour lines representing the observed relic abundance $\Omega h^{2}=0.12[20]$ as a function of dark matter couplings and $m_{Z^{\prime}}$ at four $m_{\chi}$ values. The dashed lines represent the boundary between production regimes, freeze-in and freeze-out.

3.7, Boltzmann suppression for this process happens around the $Z^{\prime}$ mass hence lower values of $m_{Z^{\prime}}$ result in $R_{Z^{\prime} \rightarrow \chi \chi}$ to be significant for longer. Notice that, since the final relic abundance is inversely proportional to the $Z^{\prime}$ mass, larger couplings are required to maintain the correct relic abundance as $m_{Z^{\prime}}$ is increased. For these reasons the correct relic abundance curve in the freeze-in regime (red) has a positive slope. In the freeze-out regime the slope is positive as well, but larger rate densities result in a lower final abundance so a larger $m_{Z^{\prime}}$ means lower rates thus larger abundance, therefore larger couplings are required.

Since we are interested in the observed abundance, Figure 5.6 shows the correct relic abundance as a function of dark matter axial and vector couplings (where $A_{\chi}=$ 
$\left.V_{\chi}\right)$ and $m_{Z^{\prime}}$ for four dark matter masses. The boundaries between the production regimes freeze-in and freeze-out are plotted as dashed lines, each colour corresponding to a different dark matter mass. The abrupt drop of the freeze-in curves at $m_{Z^{\prime}}=2 m_{\chi}$ is caused by the $Z^{\prime}$ decay becoming kinematically allowed, since the addition of this process produces more dark matter so a lower coupling is required to obtain the correct abundance. Similarly, the regime boundaries experience a sudden drop when the decay becomes allowed since it becomes easier for the dark matter to thermalize with the additional production. The troughs in the freeze-out curves at $m_{Z^{\prime}}=2 m_{\chi}$ occur because of the resonance enhancement of the s-channel process. When the schannel resonance is positioned at a temperature near dark matter freeze-out, the relic abundance drops significantly since the annihilation rate increases at the resonance. Therefore a smaller coupling is required to be able to produce the observed abundance. The slopes of the freeze-out curves are larger than the freeze-in slopes since at large couplings, the $Z^{\prime} Z^{\prime} \leftrightarrow \chi \chi$ process dominates and $R_{Z^{\prime} Z^{\prime} \leftrightarrow \chi \chi} \propto \frac{1}{m_{Z^{\prime}}^{4}}$, so a larger $Z^{\prime}$ mass requires an even larger coupling to be able to produce the observed abundance. In contrast, for the freeze-in curves the relevant processes are the s-channel and decay which are inversely proportional to a smaller power. The differences between the contours for each value of $m_{\chi}$ is simply due to the $m_{\chi}$ dependence of the relic abundance. In addition, the decay becomes allowed for $m_{Z^{\prime}}>2 m_{\chi}$. However, the regime boundaries are not so dependent on $m_{\chi}$ which is why all of the boundaries meet for $m_{Z^{\prime}}>2 m_{\chi}$. For the freeze-in portion of Figure 5.6, it is evident that the relic abundance is proportional to the dark matter couplings and inversely proportional to $m_{Z^{\prime}}$, although the effect of the dark matter couplings balance with the inverse proportionality of $m_{Z^{\prime}}$ on the part of the freeze-in curves where $m_{Z^{\prime}}<2 m_{\chi}$, so there is little $m_{Z^{\prime}}$ dependence. 


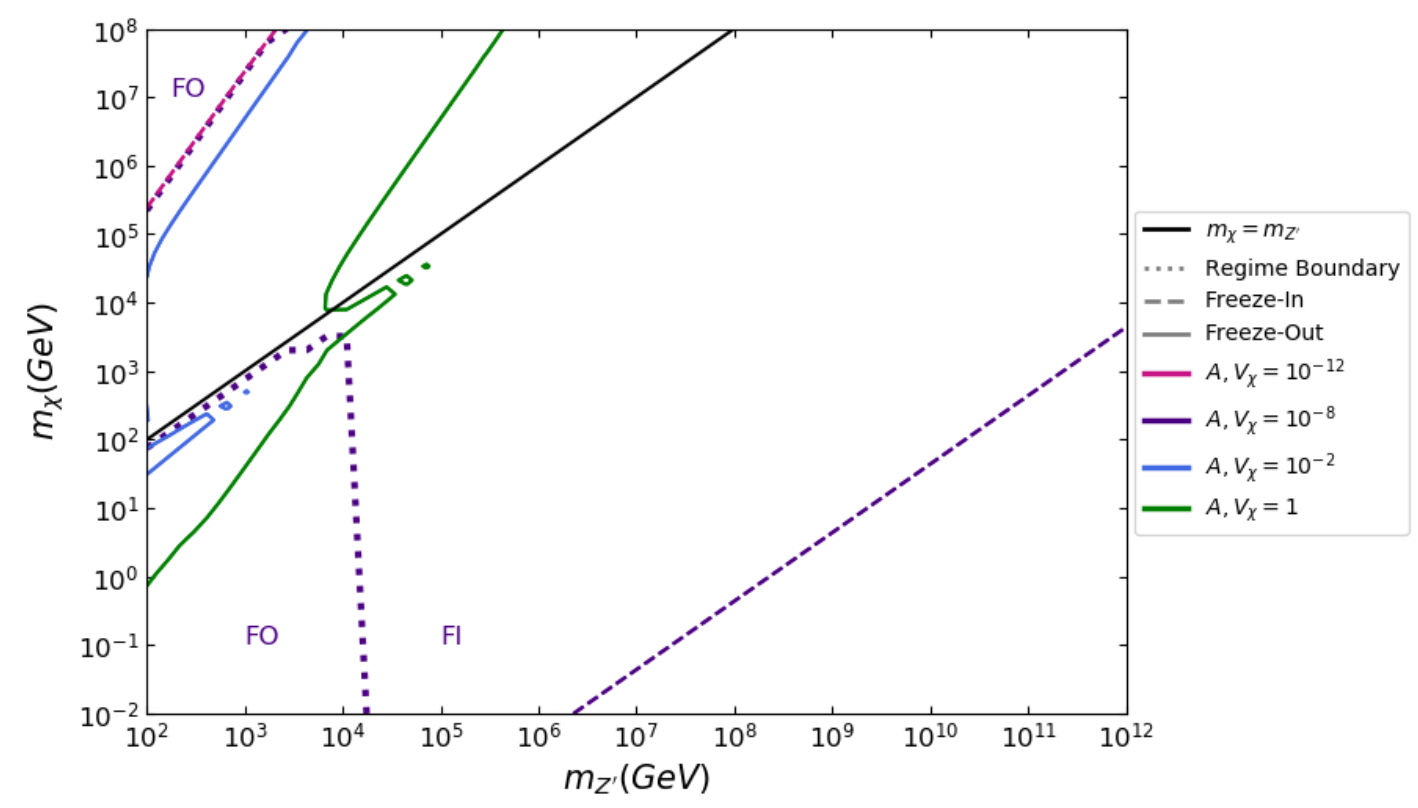

Figure 5.7: Contour lines representing the observed relic abundance $\Omega h^{2}=0.12[20]$ as a function of $m_{\chi}$ and $m_{Z^{\prime}}$ for various dark matter couplings, $A_{\chi}$ and $V_{\chi}$ where $A_{\chi}=V_{\chi}$, each represented by a different colour. The observed abundance in the freeze-in regime is represented as dashed lines, while the observed abundance in the freeze-out regime is represented by solid lines. The regime boundaries are plotted in dotted lines. The only coupling which has a regime boundary in this parameter space is $10^{-8}$, shown as purple dotted lines while each regime is labeled by purple text. The rest of the couplings are either entirely in the freeze-in or freeze-out regime.

Next, we further explore the parameter space and observe the effect $m_{\chi}$ has on these contours of observed relic abundance. Figure 5.7 shows the contour lines at the correct relic abundance as a function of $m_{\chi}$ and $m_{Z^{\prime}}$ at varying couplings. Each colour represents a different coupling, where each coupling has its own regime boundaries. At large couplings $\left(A_{\chi}, V_{\chi}=1\right.$ and $\left.A_{\chi}, V_{\chi}=10^{-2}\right)$, dark matter is sure to thermalize with the bath and is produced through freeze-out for the entire parameter space. In contrast, at low couplings $\left(A_{\chi}, V_{\chi}=10^{-12}\right)$ dark matter does not thermalize with the bath, therefore the freeze-in regime spans the entire parameter space. For mid-range couplings $\left(A_{\chi}, V_{\chi}=10^{-8}\right)$ each production regime exists in 
the plot. The observed relic abundance contour for $A_{\chi}, V_{\chi}=10^{-12}$ (magenta) falls in the part of the parameter space where the decay process is not allowed, since $2 m_{\chi}>m_{Z^{\prime}} \cdot m_{\chi}$ does not impact the rate densities significantly, although $\Omega h^{2} \propto m_{\chi}$ which is why larger dark matter masses are required to compensate for larger $Z^{\prime}$ masses since the rate densities are inversely proportional to $m_{Z^{\prime}}$. This reasoning also applies to the $A_{\chi}, V_{\chi}=10^{-8}$ curve (purple), but the coupling is larger so larger $m_{Z^{\prime}}$ and smaller $m_{\chi}$ values are required. The regime boundary corresponding to $A_{\chi}, V_{\chi}=10^{-8}$ sharply divides the parameter space near $m_{Z^{\prime}}=10^{4} \mathrm{GeV}$ for lower dark matter masses since the dominant process, $Z^{\prime} \rightarrow \chi \chi$, Boltzmann suppresses too early for sufficient production if $m_{Z^{\prime}} \gtrsim 10^{4} \mathrm{GeV}$ for this case. Thermalization is attained in the top left corner of Figure 5.7 since the $Z^{\prime} Z^{\prime} \rightarrow \chi \chi$ process has a large rate at $T_{R}$ at low $m_{Z^{\prime}}$ and large $m_{\chi}$. The $A_{\chi}, V_{\chi}=1$ (green) contour changes in behaviour near $2 m_{\chi}=m_{Z^{\prime}}$ since the decay is not allowed above the black curve and because of the s-channel's resonance enhancement. This resonance enhancement leads to more annihilation of dark matter, therefore smaller rates are needed when $m_{\chi} \approx m_{Z^{\prime}}$ hence larger $Z^{\prime}$ masses are required. Similar to the freeze-in curves, the slope of the green curve is positive away from the black curve since the rates are inversely proportional to $m_{Z^{\prime}}$ (to some power depending on the process) and $\Omega h^{2}$ is proportional to $m_{\chi}$. The $A_{\chi}, V_{\chi}=10^{-2}$ curve (blue) is very similar to the green curve but shifted over since the couplings are smaller, therefore a smaller $m_{Z^{\prime}}$ is required to produce the correct amount of dark matter and compensate for the smaller couplings.

As in Ref. [104], setting the dark matter vector coupling, $V_{\chi}$, to zero leaves us only with the axial coupling, $A_{\chi}$, which is very similar to Majorana fermion dark matter, rather than Dirac fermion dark matter ${ }^{12}$. Figure 5.8 plots the correct relic

\footnotetext{
${ }^{12}$ However, since Majorana fermions are identical particles there would be additional statistical factors in the squared amplitudes. For simplicity we set the vector coupling to zero and acknowledge that this is not exactly the Majorana case.
} 


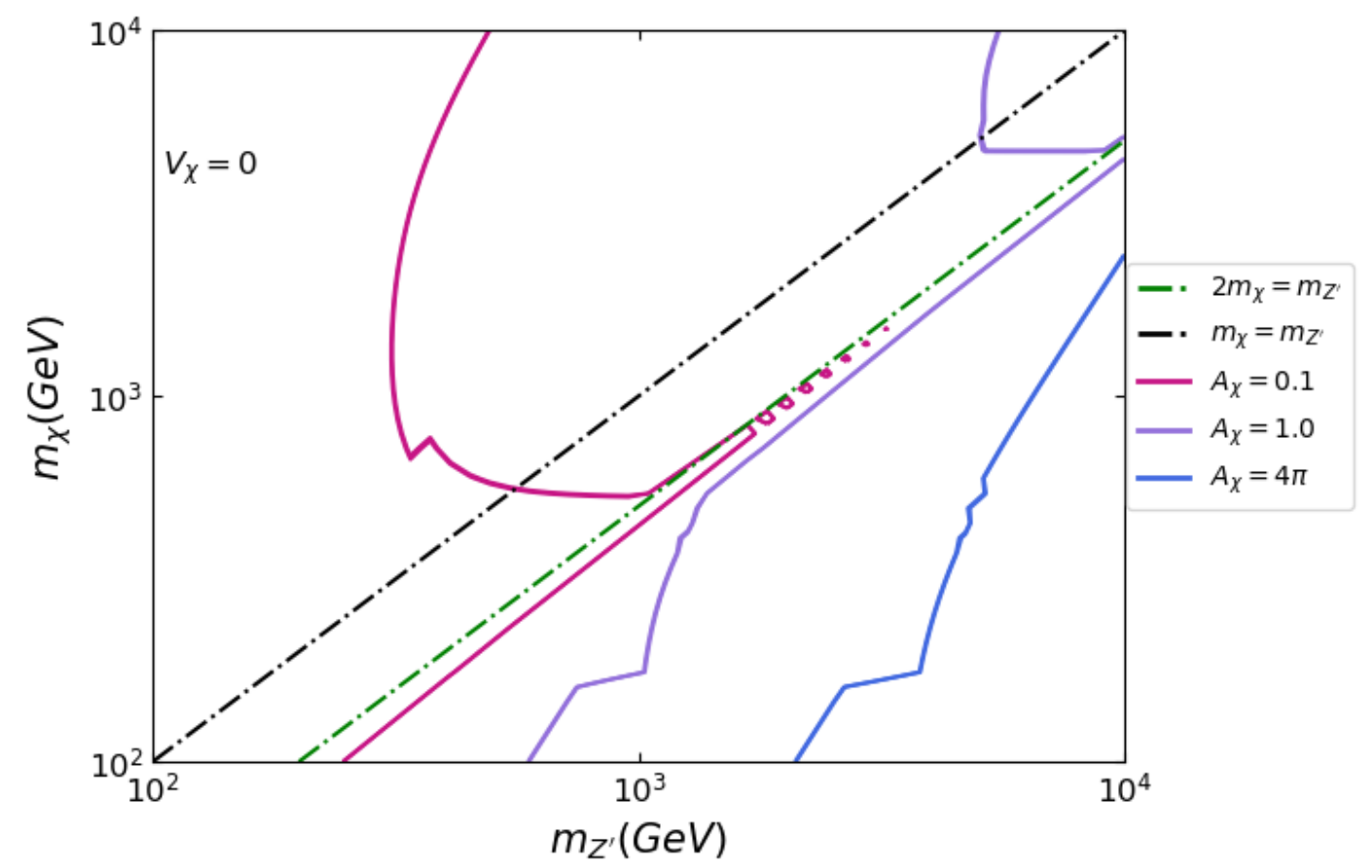

Figure 5.8: The contour lines of the correct relic abundance $\Omega h^{2}=0.12[20]$ as a function of $m_{\chi}$ and $m_{Z^{\prime}}$ for the case of $V_{\chi}=0$ [104]. Each colour represented a different Axial dark matter coupling. $m_{\chi}=m_{Z^{\prime}}$ is shown in black, and $2 m_{\chi}=m_{Z^{\prime}}$ is plotted in green. Couplings are large enough such that the entire parameter space is in the freeze-out regime.

abundance as a function of $m_{\chi}$ and $m_{Z^{\prime}}$ at three different axial couplings. All couplings in this Figure are sufficiently large such that dark matter is produced through freeze-out for the entire parameter space. The resonance enhancement of the annihilation into Standard Model fermions (where $m_{Z^{\prime}} \approx 2 m_{\chi}$ ) produces some of the observed abundance for $A_{\chi}=0.1$. The observed abundance is also produced in the region where $m_{\chi}>m_{Z^{\prime}}$, where for larger $\chi$ masses the slope is positive since the rates increase with $m_{\chi}$, as evident by equations 4.10 and 4.12 , thus larger $m_{\chi}$ values are required for larger $Z^{\prime}$ masses since the rates are inversely proportional to $m_{Z^{\prime}}$. The $A_{\chi}=1.0$ curve (violet) is similar to the pink curve although it is shifted since the coupling is larger, thus larger $Z^{\prime}$ masses are required. For $A_{\chi}=4 \pi$ (blue), $\chi \chi \rightarrow Z^{\prime} Z^{\prime}$ 
is very unlikely since the observed abundance falls in the $m_{Z^{\prime}}>m_{\chi}$ regime. 


\section{Chapter 6}

\section{Conclusion}

In this Chapter, we will first discuss a few different directions that we can take in the future for this project, and then we will summarize our findings and important points to take away from this work.

\subsection{Future Directions}

An obvious next step for this project is to examine the implications that constraints set by current experiments and constraints to be set by future experiments have on our model. These experiments include direct detection, indirect detection, and colliders. For direct detection experiments, the relevant process is $\chi q \rightarrow \chi q$, where the Feynman diagram is shown in the right subfigure of Figure 4.3, dark matter scattering off quarks in nucleons through the t-channel with $Z^{\prime}$ exchange. Experiments such as Pico-60 [117], PANDA-X [118], LUX [119] and XENON1T [51] have set constraints on the direct detection cross section. In contrast, indirect detection probes dark matter annihilation into standard model. The Fermi-LAT collaboration [120] has set indirect

detection constraints on the annihilation cross section using assumptions about the 
dark matter halo in the Milky Way. In addition, collider experiments search for new particles such as dark matter or the $Z^{\prime}$. Monojet, dijet, and dilepton limits from the LHC set bounds on the mass and branching ratio of the Sequential Standard Model $Z^{\prime}$ and dark matter $[110,121]$. It will be interesting to see if these experiments are able to rule out certain regions of the freeze-out parameter space while leaving the freeze-in regime intact. It is likely that the sequential $Z^{\prime}$ WIMP regime is already ruled out for $\mathrm{GeV}-\mathrm{TeV}$ dark matter masses, thus sub-GeV masses should be investigated.

As discussed in section 2.3, it may prove useful to track the energy transfer and temperature of the dark sector to see if dark freeze-out or reannihilation occurs due to thermalization within the dark sector. Considering this would not alter the boundary between freeze-out and freeze-in, but could potentially lead to different dark matter abundances for certain parameters in the freeze-in regime. There is a possibility of the dark matter thermalizing with itself through the $\chi \chi \rightarrow \chi \chi \chi \chi$ interaction or $\chi \chi \rightarrow \chi \chi$ t or s-channel interaction. The former is heavily suppressed since its interaction rate density would be proportional to $\lambda^{8}$ where $\lambda$ is the coupling (either $A_{\chi}, V_{\chi}$ or some combination). If the couplings are large enough such that the latter process is efficient, $\chi$ could therefore thermalize with $Z^{\prime}$ and be a part of the entire heat bath. Therefore, the only possibility of having a dark sector heat bath, which is separate from the Standard Model bath, could potentially be with a light $Z^{\prime}$ since the cross section is inversely proportional to $m_{Z^{\prime}}$. The problem is that it may be difficult to have couplings sufficiently low such that dark matter does not thermalize with the bath, but high enough that it thermalizes with itself. Throughout this thesis, the couplings to Standard Model $\left(A_{f}\right.$ and $\left.V_{f}\right)$ are fixed to the sequential values, the $Z^{\prime}$ always thermalizes with the other species thus it is always part of the Standard Model heat bath. Given that $A_{f}$ and $V_{f}$, the couplings between Standard 
Model fermions and $Z^{\prime}$, are fixed at the sequential values (Table 4.1) this assumption is justified since the couplings are large. For a different $Z^{\prime}$ model it would be best to track the evolution of $Z^{\prime}$ by solving coupled Boltzmann equations for $n_{Z^{\prime}}$ and $n_{\chi}$ to check if it freezes-out and at what temperature.

Rather than only considering the $Z^{\prime}$ portal, there exist other viable portals such as the $W^{\prime}$ boson [122], the Higgs portal [123], or neutrino portals [54]. These other mediators would involve completely different processes for dark matter production. In addition, dark matter does not have to be a fermion and could alternatively be a scalar [124] or a boson. It would be interesting to examine these other cases.

\subsection{Concluding Remarks}

In this thesis we have seen that there is undeniable evidence for the existence of dark matter, which shows itself in multiple scales of the universe such as in galaxies, clusters of galaxies, large scale structure, and the CMB. Most of the literature assumes that dark matter is a new type of particle since observations such as of the Bullet Cluster [11] show a differentiation between the visible matter and this new (dark) matter. Dark matter cannot be an already discovered particle since the only long lived and neutral species we know is the neutrino and it is not cold, i.e., it is relativistic at the time of structure formation. There exists a vast array of reasonable dark matter candidates each with many possibilities for their masses and couplings. In particular, this thesis focuses on the classes of dark matter candidates WIMPs and FIMPs, where they are produced through the freeze-out and freeze-in mechanisms respectively. These two dark matter production mechanisms are described in Chapter 2. It is clear that the thermal history of dark matter immensely impacts the final relic abundance, therefore determining which mechanism produces dark matter is 
crucial to understanding its nature. Searches for thermally produced WIMPs have yet to be successful, therefore it is important to explore alternative candidates such as FIMPs. FIMPs couple too weakly to Standard Model species to thermalize with them, so they are produced through the freeze-in mechanism and have the potential to evade certain experimental constraints. The methods to calculate the evolution of the dark matter number density in each production regime are derived in Chapter 3. In addition, we describe the way in which we differentiate between the different production mechanisms, freeze-in and freeze-out, at the beginning of Chapter 5. In order to investigate the production regimes and the boundary between them, we chose a benchmark model where dark matter interacts with Standard Model fermions directly through a sequential $Z^{\prime}$ portal. Our model is defined in Chapter 4, where we go over the interaction lagrangian and the processes. Each process that arises from our model that changes the number of dark matter particles is dominant at particular temperatures and model parameters. In Chapter 5 we explore when each process is important and why. Given these processes, the evolution of dark matter was calculated for each production mechanism and the effect of the model parameters on the evolution was discussed. Next, the final relic abundance was calculated as a function of dark matter couplings and $m_{Z^{\prime}}$ in the form of a production regime contour diagram showing the boundary between freeze-in and freeze-out. The contours of the observed abundance were found as a function of dark matter couplings, $m_{Z^{\prime}}$, and $m_{\chi}$, where the observed abundance can be achieved by both freeze-in and freeze-out. The dark matter couplings and $m_{Z^{\prime}}$ have the most influence on which production mechanism dark matter is produced through, although as shown in certain scenarios, the dark matter mass also can have an effect. In addition, the case of setting the dark matter vector coupling to zero was explored briefly in order to investigate the 
Majorana fermion case. All of these computations were done using Python code which I wrote, that calculated the interaction rates for each process and solved the Boltzmann equation in each production regime, freeze-in and freeze-out, for a given set of parameters, in order to study the dark matter production mechanisms.

As discussed in section 6.1, there is so much more that can be done with the situation presented here as well as similar scenarios. We wish to motivate the study of variations of our model in addition to different mechanisms for producing dark matter. Usually the thermalization condition is not checked, which as shown in this thesis is problematic since the dark sector does not always thermalize with the visible sector. In some cases thermalization within the dark sector could greatly affect dark matter production. My code, which differentiates between production regimes by drawing a boundary between them, is able to easily adapt to other possible scenarios. For example, sub-GeV dark matter was explored in this thesis and experiments are becoming more sensitive to it, therefore in the near future they will be more relevant. It is crucial to consider this production regime boundary on sub-GeV dark matter (or any type of dark matter) since as we have seen, the boundary is dependent on the parameters of the model. The take away from this work is that the thermal history of dark matter is extremely important to understand while exploring various dark matter models. 


\section{Appendix A}

\section{Amplitudes and Cross Sections}

Expressions for the amplitudes and cross sections for all three dark matter production processes that arise from our model are given in this Appendix.

\section{A.1 $f f \leftrightarrow \chi \chi$}

The amplitude for the process $f f \leftrightarrow \chi \chi$ is given by,

$$
M=\frac{-1}{s-m_{Z^{\prime}}^{2}+i \Gamma_{Z^{\prime}}} \bar{u}(4) i \gamma^{\mu}\left(V_{\chi}-A_{\chi} \gamma^{5}\right) v(3) i\left(g_{\mu \nu}-\frac{q_{\mu} q_{\nu}}{m_{Z^{\prime}}^{2}}\right) \bar{v}(2) i \gamma^{\nu}\left(V_{f}-A_{f} \gamma^{5}\right) u(1)
$$


where $s$ is the Mandelstam variable (not the entropy density). The squared amplitude averaged over initial and final spins is then,

$$
\begin{aligned}
\left|\bar{M}^{2}\right| & =\frac{s^{2}}{16\left[\left(s-m_{Z^{\prime}}^{2}\right)^{2}+m_{Z^{\prime}}^{2} \Gamma_{Z^{\prime}}^{2}\right]}\left[V_{f}^{2} V_{\chi}^{2}\left\{1+\frac{4 m_{f}^{2}}{s}+\frac{4 m_{\chi}^{2}}{s}\right\}\right. \\
& +V_{f}^{2} A_{\chi}^{2}\left\{1-\frac{4 m_{\chi}^{2}}{s}+\frac{4 m_{f}^{2}}{s}-\frac{16 m_{f}^{2} m_{\chi}^{2}}{s^{2}}\right\} \\
& +A_{f}^{2} V_{\chi}^{2}\left\{1-\frac{4 m_{f}^{2}}{s}+\frac{4 m_{\chi}^{2}}{s}-\frac{16 m_{f}^{2} m_{\chi}^{2}}{s^{2}}\right\} \\
& +A_{f}^{2} A_{\chi}^{2}\left\{\frac{16 m_{f}^{2} m_{\chi}^{2}}{m_{Z^{\prime}}^{4}}+\frac{32 m_{f}^{2} m_{\chi}^{2}}{s^{2}}-\frac{4 m_{f}^{2}}{s}-\frac{32 m_{f}^{2} m_{\chi}^{2}}{s m_{Z^{\prime}}^{2}}-\frac{4 m_{\chi}^{2}}{s}+1\right\} \\
& +\cos ^{2} \theta\left(A_{f}^{2}+V_{f}^{2}\right)\left(A_{\chi}^{2}+V_{\chi}^{2}\right)\left(\frac{16 m_{f}^{2} m_{\chi}^{2}}{s^{2}}-\frac{4\left(m_{f}^{2}+m_{\chi}^{2}\right)}{s}+1\right) \\
& \left.-8 \cos \theta A_{f} A_{\chi} V_{f} V_{\chi} \sqrt{1-\frac{4 m_{f}^{2}}{s}} \sqrt{1-\frac{4 m_{\chi}^{2}}{s}}\right] .
\end{aligned}
$$

The cross section is given by,

$$
\begin{aligned}
\sigma_{f f \rightarrow \chi \chi} & =\frac{\sqrt{s-4 m_{\chi}^{2}}}{48 s \pi \sqrt{s-4 m_{f}^{2}}\left(\left(s-m_{Z^{\prime}}^{2}\right)^{2}+m_{Z^{\prime}}^{2} \Gamma_{Z^{\prime}}^{2}\right)}\left\{A _ { f } ^ { 2 } \left[A _ { \chi } ^ { 2 } \left(\frac{12 m_{f}^{2} m_{\chi}^{2} s^{2}}{m_{Z^{\prime}}^{4}}-\frac{24 m_{f}^{2} m_{\chi}^{2} s}{m_{Z^{\prime}}^{2}}\right.\right.\right. \\
& \left.\left.+28 m_{f}^{2} m_{\chi}^{2}-4 s m_{f}^{2}+s^{2}-4 s m_{\chi}^{2}\right)-V_{\chi}^{2}\left(8 m_{f}^{2} m_{\chi}^{2}+4 s m_{f}^{2}-2 s m_{\chi}^{2}-s^{2}\right)\right] \\
& \left.+V_{f}^{2}\left(2 m_{f}^{2}+s\right)\left(A_{\chi}^{2} s-4 A_{\chi}^{2} m_{\chi}^{2}+2 V_{\chi}^{2} m_{\chi}^{2}+V_{\chi}^{2} s\right)\right\} .
\end{aligned}
$$

The cross section for the reverse process, $\chi \chi \rightarrow f f$ follows similarly.

\section{A.2 $Z^{\prime} \leftrightarrow f f / \chi \chi$}

Next, for the process $Z^{\prime} \leftrightarrow f f / \chi \chi$ the amplitude is,

$$
M=\epsilon_{\mu}(1) \bar{u}(3) i \gamma^{\mu}\left(V_{\chi / f}-A_{\chi / f} \gamma^{5}\right) v(2)
$$


For the squared amplitude, we will average over initial and sum over finals spins and include the additional final spin factors when computing the rate densities,

$$
\left|\bar{M}^{2}\right|=\frac{4}{3} m_{Z^{\prime}}^{2}\left[V_{\chi / f}^{2}\left(1+\frac{2 m_{\chi / f}^{2}}{m_{Z^{\prime}}^{2}}\right)+A_{\chi / f}^{2}\left(1-\frac{4 m_{\chi / f}^{2}}{m_{Z^{\prime}}^{2}}\right)\right]
$$

\section{A.3 $Z^{\prime} Z^{\prime} \leftrightarrow \chi \chi$}

Finally we have the $Z^{\prime} Z^{\prime} \rightarrow \chi \chi$ process where the amplitude is given by,

$$
\begin{aligned}
M & =\frac{1}{t-m_{\chi}^{2}} \bar{u}(4) i \gamma^{\mu}\left(V_{\chi}-A_{\chi} \gamma^{5}\right) i\left(p_{3}-\not p_{1}+m_{\chi}\right) i \gamma^{\nu}\left(V_{\chi}-A_{\chi} \gamma^{5}\right) v(3) \epsilon_{\mu}(2) \epsilon_{\nu}(1) \\
& +\frac{1}{u-m_{\chi}^{2}} \bar{u}(4) i \gamma^{\nu}\left(V_{\chi}-A_{\chi} \gamma^{5}\right) i\left(\not p_{1}-\not p_{4}+m_{\chi}\right) i \gamma^{\mu}\left(V_{\chi}-A_{\chi} \gamma^{5}\right) v(3) \epsilon_{\mu}(2) \epsilon_{\nu}(1)
\end{aligned}
$$

and the squared amplitudes summed over spins are,

$$
\begin{aligned}
&\left|M_{t t}\right|^{2}=\left[\frac{1}{t-m_{\chi}^{2}}\right]^{2} \operatorname{Tr}\left[\left(p_{3}+m_{\chi}\right) \gamma^{\mu}\left(V_{\chi}-A_{\chi} \gamma^{5}\right)\left(p_{3}-\not p_{1}+m_{\chi}\right)\right. \\
&\left.\gamma^{\nu}\left(V_{\chi}-A_{\chi} \gamma^{5}\right)\left(p_{4}-m_{\chi}\right)\left(\gamma^{\beta}\left(V_{\chi}-A_{\chi} \gamma^{5}\right)\left(p_{3}-\not p_{1}+m_{\chi}\right) \gamma^{\alpha}\left(V_{\chi}-A_{\chi} \gamma^{5}\right)\right)^{*}\right] \\
&\left(g_{\mu \alpha}-\frac{p_{2 \mu} p_{2 \alpha}}{m_{Z^{\prime}}^{2}}\right)\left(g_{\nu \beta}-\frac{p_{1 \nu} p_{1 \beta}}{m_{Z^{\prime}}^{2}}\right)\left(\gamma_{Z^{\prime}}^{2} \gamma_{\chi}^{2}\right)^{-1} \\
&\left|M_{u u}\right|^{2}=\left[\frac{1}{u-m_{\chi}^{2}}\right]^{2} \operatorname{Tr}\left[\left(p_{3}+m_{\chi}\right) \gamma^{\nu}\left(V_{\chi}-A_{\chi} \gamma^{5}\right)\left(p_{1}-\not p_{4}+m_{\chi}\right)\right. \\
&\left.\gamma^{\mu}\left(V_{\chi}-A_{\chi} \gamma^{5}\right)\left(p_{4}-m_{\chi}\right)\left(\gamma^{\alpha}\left(V_{\chi}-A_{\chi} \gamma^{5}\right)\left(p_{1}-\not p_{4}+m_{\chi}\right) \gamma^{\beta}\left(V_{\chi}-A_{\chi} \gamma^{5}\right)\right)^{*}\right] \\
&\left(g_{\mu \alpha}-\frac{p_{2 \mu} p_{2 \alpha}}{m_{Z^{\prime}}^{2}}\right)\left(g_{\nu \beta}-\frac{p_{1 \nu} p_{1 \beta}}{m_{Z^{\prime}}^{2}}\right)\left(\gamma_{Z^{\prime}}^{2} \gamma_{\chi}^{2}\right)^{-1}
\end{aligned}
$$




$$
\begin{aligned}
& \left|M_{t u}\right|^{2}=\left[\frac{1}{t-m_{\chi}^{2}}\right]\left[\frac{1}{u-m_{\chi}^{2}}\right] \operatorname{Tr}\left[\left(p_{3}+m_{\chi}\right) \gamma^{\mu}\left(V_{\chi}-A_{\chi} \gamma^{5}\right)\left(p_{3}-\not p_{1}+m_{\chi}\right)\right. \\
& \left.\gamma^{\nu}\left(V_{\chi}-A_{\chi} \gamma^{5}\right)\left(\not p_{4}-m_{\chi}\right)\left(\gamma^{\alpha}\left(V_{\chi}-A_{\chi} \gamma^{5}\right)\left(\not p_{1}-\not p_{4}+m_{\chi}\right) \gamma^{\beta}\left(V_{\chi}-A_{\chi} \gamma^{5}\right)\right)^{*}\right] \\
& \left(g_{\mu \alpha}-\frac{p_{2 \mu} p_{2 \alpha}}{m_{Z^{\prime}}^{2}}\right)\left(g_{\nu \beta}-\frac{p_{1 \nu} p_{1 \beta}}{m_{Z^{\prime}}^{2}}\right)\left(\gamma_{Z^{\prime}}^{2} \gamma_{\chi}^{2}\right)^{-1} \\
& |M|^{2}=2\left|M_{t u}\right|^{2}+\left|M_{u u}\right|^{2}+\left|M_{t t}\right|^{2}=\frac{M_{n u m}^{2}}{m_{Z^{\prime}}^{4}\left(\cos ^{2}(\theta)\left(s-4 m_{\chi}^{2}\right)\left(4 m_{Z^{\prime}}^{2}-s\right)+\left(s-2 m_{Z^{\prime}}^{2}\right)^{2}\right)^{2}} \\
& M_{n u m}^{2}=16\left(\cos ^{4}(\theta)\left(s-4 m_{\chi}^{2}\right)^{2}\left(-\left(s-4 m_{Z^{\prime}}^{2}\right)^{2}\right)\right. \\
& \left(A_{\chi}^{4}\left(-2 m_{\chi}^{2} s+m_{Z^{\prime}}^{4}+8 m_{\chi}^{2} m_{Z^{\prime}}^{2}\right) 6 A_{\chi}^{2} m_{Z^{\prime}}^{4} V_{\chi}^{2}+m_{Z^{\prime}}^{4} V_{\chi}^{4}\right) \\
& +\left(s-2 m_{Z^{\prime}}^{2}\right)^{2}\left(A _ { \chi } ^ { 4 } \left(2 m_{\chi}^{2} s^{2}\left(s-4 m_{\chi}^{2}\right)+4 m_{Z^{\prime}}^{6}\left(s-8 m_{\chi}^{2}\right)\right.\right. \\
& \left.+m_{Z^{\prime}}^{4}\left(-64 m_{\chi}^{4}+16 m_{\chi}^{2} s+s^{2}\right)+8 m_{\chi}^{2} m_{Z^{\prime}}^{2} s\left(4 m_{\chi}^{2}-s\right)\right) \\
& +2 A_{\chi}^{2} m_{Z^{\prime}}^{2} V_{\chi}^{2}\left(4 m_{\chi}^{2} s^{2}+12 m_{Z^{\prime}}^{4} s+m_{Z^{\prime}}^{2}\left(64 m_{\chi}^{4}-56 m_{\chi}^{2} s+3 s^{2}\right)\right) \\
& \left.+m_{Z^{\prime}}^{4} V_{\chi}^{4}\left(-32 m_{\chi}^{4}+8 m_{\chi}^{2} s+4 m_{Z^{\prime}}^{2}\left(s-8 m_{\chi}^{2}\right)+s^{2}\right)\right) \\
& +4 \cos ^{2}(\theta)\left(s-4 m_{\chi}^{2}\right)\left(4 m_{Z^{\prime}}^{2}-s\right)\left(A _ { \chi } ^ { 4 } \left(m_{\chi}^{2} s^{2}\left(s-2 m_{\chi}^{2}\right)+m_{Z^{\prime}}^{8}\right.\right. \\
& \left.+2 m_{Z^{\prime}}^{6}\left(s-16 m_{\chi}^{2}\right)+2 m_{Z^{\prime}}^{4}\left(8 m_{\chi}^{4}+7 m_{\chi}^{2} s\right)+2 m_{\chi}^{2} m_{Z^{\prime}}^{2} s\left(4 m_{\chi}^{2}-3 s\right)\right) \\
& +2 A_{\chi}^{2} m_{Z^{\prime}}^{2} V_{\chi}^{2}\left(m_{\chi}^{2} s^{2}+3 m_{Z^{\prime}}^{6}+m_{Z^{\prime}}^{4}\left(6 s-20 m_{\chi}^{2}\right)-6 m_{\chi}^{2} m_{Z^{\prime}}^{2} s\right) \\
& \left.\left.+m_{Z^{\prime}}^{4} V_{\chi}^{4}\left(m_{Z^{\prime}}^{4}+2 s\left(m_{\chi}^{2}+m_{Z^{\prime}}^{2}\right)\right)\right)\right) \text {. }
\end{aligned}
$$

Note that the interference term $M_{t u}$ is added rather than subtracted since there is no fermion exchange between the $t$ and $u$ channel diagrams. The differential cross section is written in terms of the squared amplitude,

$$
\frac{d \sigma_{12 \rightarrow 34}}{d \Omega}=\frac{S_{f} p_{1}}{64 \pi^{2} s p_{3}}|\bar{M}|_{12 \rightarrow 34}^{2}
$$

where $S_{f}$ is a statistical factor accounting for identical particles, in this case $S_{f}=\frac{1}{2}$ to account for the two $Z^{\prime}$ particles. In cosmology we must account for identical particles 
in the final and initial state, since the integration of the initial and final phase space double counts these identical cases. 


\section{References}

[1] G. Aad, T. Abajyan, B. Abbott, J. Abdallah, S. Abdel Khalek, A. Abdelalim et al., Observation of a new particle in the search for the standard model higgs boson with the atlas detector at the lhc, Physics Letters B 716 (2012) 1-29.

[2] S. Chatrchyan, V. Khachatryan, A. Sirunyan, A. Tumasyan, W. Adam, E. Aguilo et al., Observation of a new boson at a mass of 125 gev with the cms experiment at the lhc, Physics Letters B 716 (2012) 30-61.

[3] C. Campagnari and M. Franklin, The discovery of the top quark, Rev. Mod. Phys. 69 (1997) 137.

[4] P. Watkins, DISCOVERY OF THE W AND Z BOSONS, Contemp. Phys. 27 (1986) 291.

[5] B. Allanach, Beyond the Standard Model Lectures for the 2016 European School of High-Energy Physics, in 2016 European School of High-Energy Physics, pp. 123-152, 2017, DOI [1609.02015].

[6] J. MUSSELL, Arthur cowper ranyard, knowledge and the reproduction of astronomical photographs in the late nineteenth-century periodical press, The British Journal for the History of Science 42 (2009) 345.

[7] XII. extract from the translation of a letter from professor bessel, dated konigsberg, 10th of august, 1844. on the variations of the proper motions of procyon and sirius, Monthly Notices of the Royal Astronomical Society 6 (1844) 136.

[8] G. Bertone and D. Hooper, A history of dark matter, arXiv:1605.04909.

[9] W. Thomson, Baron Kelvin, Baltimore Lectures on Molecular Dynamics and the Wave Theory of Light, Cambridge Library Collection - Physical Sciences. Cambridge University Press, 2010, 10.1017/CBO9780511694523.

[10] H. Andernach and F. Zwicky, English and spanish translation of zwicky's (1933) the redshift of extragalactic nebulae, arXiv:1711.01693. 
[11] A. Robertson, R. Massey and V. Eke, What does the bullet cluster tell us about self-interacting dark matter?, arXiv:1605.04307.

[12] $1 E$ 0657-56. NASA.

[13] D. Clowe, A. Gonzalez and M. Markevitch, Weak lensing mass reconstruction of the interacting cluster 1e0657-558: Direct evidence for the existence of dark matter, arXiv:astro-ph/0312273.

[14] K. Begeman, A. Broeils and R. Sanders, Extended rotation curves of spiral galaxies: Dark haloes and modified dynamics, Mon. Not. Roy. Astron. Soc. 249 (1991) 523.

[15] G. Bertone, D. Hooper and J. Silk, Particle dark matter: Evidence, candidates and constraints, arXiv:hep-ph/0404175.

[16] R. A. Flores and J. R. Primack, Observational and theoretical constraints on singular dark matter halos, arXiv:astro-ph/9402004.

[17] R. Brada and M. Milgrom, Exact solutions and approximations of mond fields of disk galaxies, arXiv:astro-ph/9407071.

[18] D. Scott, M. White, J. D. Cohn and E. Pierpaoli, Cosmological difficulties with modified newtonian dynamics (or: La fin du mond?), arXiv: astro-ph/0104435.

[19] C. S. Frenk and S. D. M. White, Dark matter and cosmic structure, arXiv: 1210.0544.

[20] P. Collaboration, N. Aghanim, Y. Akrami, M. Ashdown, J. Aumont, C. Baccigalupi et al., Planck 2018 results. vi. cosmological parameters, arXiv: 1807.06209.

[21] Planck CMB. ESA, Mar, 2013.

[22] W. Hu and S. Dodelson, Cosmic microwave background anisotropies, arXiv: astro-ph/0110414.

[23] P. Bull et al., Beyond $\Lambda$ CDM: Problems, solutions, and the road ahead, Phys. Dark Univ. 12 (2016) 56 [1512.05356].

[24] P. J. E. Peebles, Primeval adiabatic perturbations - effect of massive neutrinos, The Astrophysical Journal 258 (1982) 415.

[25] S. D. M. White, C. S. Frenk and M. Davis, Clustering in a neutrino-dominated universe, The Astrophysical Journal 274 (1983) L1. 
[26] S. Tremaine and J. E. Gunn, Dynamical role of light neutral leptons in cosmology, Physical Review Letters 42 (1979) 407.

[27] A. Boyarsky, M. Drewes, T. Lasserre, S. Mertens and O. Ruchayskiy, Sterile neutrino dark matter, arXiv:1807.07938.

[28] H. Niikura, M. Takada, N. Yasuda, R. H. Lupton, T. Sumi, S. More et al., Microlensing constraints on primordial black holes with subaru/HSC andromeda observations, Nature Astronomy 3 (2019) 524.

[29] J. D. Simon, T. S. Li, A. Drlica-Wagner, K. Bechtol, J. L. Marshall, D. J. James et al., Nearest neighbor: The low-mass milky way satellite tucana III, The Astrophysical Journal 838 (2017) 11.

[30] B. Carr and F. Kuhnel, Primordial black holes as dark matter: Recent developments, 2006.02838.

[31] M. Milgrom, A modification of the newtonian dynamics as a possible alternative to the hidden mass hypothesis, The Astrophysical Journal $\mathbf{2 7 0}$ (1983) 365 .

[32] M. Milgrom, A modification of the newtonian dynamics - implications for galaxies, The Astrophysical Journal 270 (1983) 371.

[33] M. Milgrom, A modification of the newtonian dynamics - implications for galaxy systems, The Astrophysical Journal 270 (1983) 384.

[34] A. Aguirre, J. Schaye and E. Quataert, Problems for modified newtonian dynamics in clusters and the lya forest?, The Astrophysical Journal $\mathbf{5 6 1}$ (2001) 550 .

[35] J. H. Gundlach, S. Schlamminger, C. D. Spitzer, K.-Y. Choi, B. A. Woodahl, J. J. Coy et al., Laboratory test of newton's second law for small accelerations, Physical Review Letters 98 (2007).

[36] A. Abramovici and Z. Vager, Test of newton's second law at small accelerations, Physical Review D 34 (1986) 3240.

[37] S. Profumo, L. Giani and O. F. Piattella, An Introduction to Particle Dark Matter, Universe 5 (2019) 213 [1910.05610].

[38] L. D. Duffy and K. v. Bibber, Axions as dark matter particles, New Journal of Physics 11 (2009) 105008.

[39] R. D. Peccei and H. R. Quinn, CPConservation in the presence of pseudoparticles, Physical Review Letters 38 (1977) 1440. 
[40] R. D. Peccei and H. R. Quinn, Constraints imposed byCPconservation in the presence of pseudoparticles, Physical Review D 16 (1977) 1791.

[41] S. Weinberg, A new light boson?, Physical Review Letters 40 (1978) 223.

[42] F. Wilczek, Problem of StrongPandTInvariance in the presence of instantons, Physical Review Letters 40 (1978) 279.

[43] K. Saikawa and S. Shirai, Precise wimp dark matter abundance and standard model thermodynamics, arXiv:2005.03544.

[44] M. Schumann, Direct detection of wimp dark matter: Concepts and status, arXiv: 1903.03026

[45] G. Arcadi, M. Dutra, P. Ghosh, M. Lindner, Y. Mambrini, M. Pierre et al., The waning of the wimp? a review of models, searches, and constraints, arXiv: 1703.07364.

[46] L. Roszkowski, E. M. Sessolo and S. Trojanowski, Wimp dark matter candidates and searches - current status and future prospects, arXiv: 1707.06277.

[47] M. Yamada, Gauge hierarchy problem and scalegenesis, arXiv:2004.00142.

[48] M. Klasen, M. Pohl and G. Sigl, Indirect and direct search for dark matter, arXiv: 1507.03800.

[49] DEAP Collaboration collaboration, Search for dark matter with a 231-day exposure of liquid argon using deap-3600 at snolab, Phys. Rev. D 100 (2019) 022004.

[50] L. Collaboration, D. S. Akerib, H. M. Araujo, X. Bai, A. J. Bailey, J. Balajthy et al., First results from the lux dark matter experiment at the sanford underground research facility, arXiv:1310.8214.

[51] E. Aprile, J. Aalbers, F. Agostini, M. Alfonsi, L. Althueser, F. D. Amaro et al., Dark matter search results from a one tonnexyear exposure of xenon1t, arXiv: 1805.12562.

[52] S. Collaboration, R. Agnese, T. Aralis, T. Aramaki, I. J. Arnquist, E. Azadbakht et al., First dark matter constraints from a supercdms single-charge sensitive detector, arXiv:1804.10697.

[53] N. Bernal, M. Heikinheimo, T. Tenkanen, K. Tuominen and V. Vaskonen, The dawn of fimp dark matter: A review of models and constraints, arXiv: 1706.07442. 
[54] C. Cosme, M. Dutra, T. Ma, Y. Wu and L. Yang, Neutrino portal to fimp dark matter with an early matter era, arXiv:2003.01723.

[55] M. Srednicki, R. Watkins and K. A. Olive, Calculations of relic densities in the early universe, Nuclear Physics B 310 (1988) 693.

[56] H. Okada, Y. Orikasa and T. Toma, Nonthermal dark matter models and signals, Physical Review D 93 (2016).

[57] H. Baer, K.-Y. Choi, J. E. Kim and L. Roszkowski, Dark matter production in the early universe: beyond the thermal wimp paradigm, arXiv:1407.0017.

[58] L. J. Hall, K. Jedamzik, J. March-Russell and S. M. West, Freeze-in production of fimp dark matter, arXiv:0911.1120.

[59] X. Chu, T. Hambye and M. H. Tytgat, The four basic ways of creating dark matter through a portal, Journal of Cosmology and Astroparticle Physics 2012 (2012) 034.

[60] K. Lozanov, "Lectures on reheating after inflation."

[61] L. Kofman, The origin of matter in the universe: Reheating after inflation, arXiv:astro-ph/9605155.

[62] N. Bernal, X. Chu, C. Garcia-Cely, T. Hambye and B. Zaldivar, Production regimes for self-interacting dark matter, Journal of Cosmology and Astroparticle Physics 2016 (2016) 018.

[63] M. Heikinheimo, T. Tenkanen, K. Tuominen and V. Vaskonen, Erratum: Observational constraints on decoupled hidden sectors [phys. rev. d 94, 063506 (2016)], Physical Review D 96 (2017) .

[64] N. Bernal and X. Chu, Z2 simp dark matter, Journal of Cosmology and Astroparticle Physics 2016 (2016) 006.

[65] N. Bernal, Boosting freeze-in through thermalization, arXiv:2005.08988.

[66] N. Bernal, X. Chu and J. Pradler, Simply split strongly interacting massive particles, Physical Review D 95 (2017).

[67] C. Cheung, G. Elor, L. J. Hall and P. Kumar, Origins of hidden sector dark matter II: collider physics, Journal of High Energy Physics 2011 (2011).

[68] C. Cheung, G. Elor, L. J. Hall and P. Kumar, Origins of hidden sector dark matter i: cosmology, Journal of High Energy Physics 2011 (2011) .

[69] T. Tenkanen and V. Vaskonen, Reheating the standard model from a hidden sector, Physical Review D 94 (2016) . 
[70] D. J. H. Chung, E. W. Kolb and A. Riotto, Superheavy dark matter, Physical Review D 59 (1998) .

[71] P. Gondolo, Introduction to non-baryonic dark matter, arXiv: astro-ph/0403064.

[72] E. W. Kolb and A. J. Long, Superheavy dark matter through higgs portal operators, Physical Review D 96 (2017).

[73] L. Parker, Quantized fields and particle creation in expanding universes. $i$, Physical Review 183 (1969) 1057.

[74] D. J. H. Chung, E. W. Kolb and A. Riotto, Production of massive particles during reheating, Physical Review D 60 (1999) .

[75] V. A. Kuzmin and I. I. Tkachev, Ultrahigh-energy cosmic rays, superheavy long-lived particles, and matter creation after inflation, Journal of Experimental and Theoretical Physics Letters 68 (1998) 271.

[76] E. W. Kolb, A. Riotto and I. I. Tkachev, GUT baryogenesis after preheating: numerical study of the production and decay of $x$-bosons, Physics Letters $B$ 423 (1998) 348.

[77] E. W. Kolb, A. Linde and A. Riotto, Grand-unified-theory baryogenesis after preheating, Physical Review Letters 77 (1996) 4290.

[78] D. J. H. Chung, E. W. Kolb and A. Riotto, Nonthermal supermassive dark matter, Physical Review Letters 81 (1998) 4048.

[79] A. Salvio, A. Strumia and W. Xue, Thermal axion production, arXiv: 1310.6982

[80] L. Abbott and P. Sikivie, A cosmological bound on the invisible axion, Physics Letters B 120 (1983) 133.

[81] R. L. Davis, Goldstone Bosons in String Models of Galaxy Formation, Phys. Rev. D 32 (1985) 3172.

[82] S. Chang, C. Hagmann and P. Sikivie, Studies of the motion and decay of axion walls bounded by strings, Physical Review D 59 (1998).

[83] O. Bertolami, C. Cosme and J. G. Rosa, Scalar field dark matter and the higgs field, arXiv: 1603.06242.

[84] C. Cosme, J. G. Rosa and O. Bertolami, Scalar field dark matter with spontaneous symmetry breaking and the 3.5 kev line, arXiv:1709.09674. 
[85] C. Cosme, J. G. Rosa and O. Bertolami, Scale-invariant scalar field dark matter through the higgs portal, arXiv:1802.09434.

[86] L. Hui, J. P. Ostriker, S. Tremaine and E. Witten, Ultralight scalars as cosmological dark matter, arXiv:1610.08297.

[87] W. Hu, R. Barkana and A. Gruzinov, Fuzzy cold dark matter: The wave properties of ultralight particles, Physical Review Letters 85 (2000) 1158-1161.

[88] A. Diez-Tejedor and D. J. E. Marsh, Cosmological production of ultralight dark matter axions, arXiv:1702.02116.

[89] J. G. Rosa and L. B. Ventura, Warm little inflaton becomes cold dark matter, Physical Review Letters 122 (2019).

[90] E. W. Kolb and M. S. Turner, The early universe, Frontiers in Physics. Westview Press, Boulder, CO, 1990, 10.1201/9780429492860.

[91] L. Husdal, On effective degrees of freedom in the early universe, arXiv: 1609.04979.

[92] P. Gondolo and G. Gelmini, Cosmic abundances of stable particles: Improved analysis, Nuclear Physics B 360 (1991) 145 .

[93] T. Binder, T. Bringmann, M. Gustafsson and A. Hryczuk, Erratum: Early kinetic decoupling of dark matter: When the standard way of calculating the thermal relic density fails [phys. rev. d 96 , 115010 (2017)], Physical Review D 101 (2020).

[94] D. Baumann, "Cosmology."

[95] R. Allahverdi, R. Brandenberger, F.-Y. Cyr-Racine and A. Mazumdar, Reheating in inflationary cosmology: Theory and applications, arXiv: 1001.2600.

[96] J. Hewett, Low-energy phenomenology of superstring-inspired e6 models, Physics Reports 183 (1989) 193.

[97] G. Arcadi, M. Lindner, Y. Mambrini, M. Pierre and F. S. Queiroz, Gut models at current and future hadron colliders and implications to dark matter searches, arXiv:1704.02328.

[98] W. Rodejohann and C. E. Yaguna, Scalar dark matter in the b-l model, arXiv: 1509.04036

[99] T. Gherghetta, J. Kersten, K. Olive and M. Pospelov, Evaluating the price of tiny kinetic mixing, Phys. Rev. D 100 (2019) 095001. 
[100] F. del Aguila, M. Masip and M. Perez-Victoria, Physical parameters and renormalization of $u(1)^{\prime}$ a $x u(1)^{\prime} b$ models, arXiv:hep-ph/9507455.

[101] K. Schmitz, Desy workshop seminar, Apr, 2020.

[102] Y. Mambrini, The zz' kinetic mixing in the light of the recent direct and indirect dark matter searches, arXiv:1104.4799.

[103] B. Holdom, Two u(1)'s and $\epsilon$ charge shifts, Physics Letters B 166 (1986) 196.

[104] G. Arcadi, M. D. Campos, M. Lindner, A. Masiero and F. S. Queiroz, The dark sequential z' portal: Collider and direct detection experiments, arXiv: 1708.00890.

[105] E. J. Chun, J.-C. Park and S. Scopel, Dark matter and a new gauge boson through kinetic mixing, arXiv:1011.3300.

[106] J. Kumar and J. D. Wells, Hadron and linear collider probes of hidden-sector gauge bosons, Phys. Rev. D 74 (2006) 115017.

[107] W.-F. Chang, J. N. Ng and J. M. S. Wu, Very narrow shadow extraZboson at colliders, Physical Review D 74 (2006).

[108] W.-F. Chang, J. N. Ng and J. M. S. Wu, A very narrow shadow extra z-boson at colliders, arXiv:hep-ph/0608068.

[109] P. Langacker, The physics of heavy z' gauge bosons, arXiv:0801.1345.

[110] A. Alves, S. Profumo and F. S. Queiroz, The dark z' portal: Direct, indirect and collider searches, arXiv:1312.5281.

[111] D. J. Griffiths, Introduction to elementary particles; 2nd rev. version, Physics textbook. Wiley, New York, NY, 2008.

[112] T. M. Tait, "Tasi lectures on resonances."

[113] F. Kahlhoefer, K. Schmidt-Hoberg, T. Schwetz and S. Vogl, Implications of unitarity and gauge invariance for simplified dark matter models, Journal of High Energy Physics 2016 (2016) .

[114] M. Duerr, F. Kahlhoefer, K. Schmidt-Hoberg, T. Schwetz and S. Vogl, How to save the WIMP: global analysis of a dark matter model with two s-channel mediators, Journal of High Energy Physics 2016 (2016) .

[115] S. Hannestad, What is the lowest possible reheating temperature?, Physical Review D 70 (2004). 
[116] P. Collaboration, P. A. R. Ade, N. Aghanim, M. Arnaud, M. Ashdown, J. Aumont et al., Planck 2015 results. xiii. cosmological parameters, arXiv: 1502.01589.

[117] PICO COllaboration collaboration, Dark matter search results from the PICO-60 c $\mathrm{f}_{8}$ bubble chamber, Phys. Rev. Lett. 118 (2017) 251301.

[118] X. Cui, A. Abdukerim, W. Chen, X. Chen, Y. Chen, B. Dong et al., Dark matter results from 54-ton-day exposure of PandaX-II experiment, Physical Review Letters 119 (2017).

[119] LUX Collaboration collaboration, Improved limits on scattering of weakly interacting massive particles from reanalysis of 2013 lux data, Phys. Rev. Lett. 116 (2016) 161301.

[120] M. Ackermann, A. Albert, B. Anderson, L. Baldini, J. Ballet, G. Barbiellini et al., Dark matter constraints from observations of 25 milky way satellite galaxies with the fermi large area telescope, Physical Review D 89 (2014) .

[121] A. Collaboration, Search for new high-mass phenomena in the dilepton final state using $36 \mathrm{fb}^{-1}$ of proton-proton collision data at $\sqrt{\mathrm{s}}=13$ tev with the atlas detector, arXiv:1707.02424.

[122] D. Duffty and Z. Sullivan, Model independent reach for w' bosons at the lhc, Physical Review D 86 (2012) .

[123] G. Arcadi, A. Djouadi and M. Raidal, Dark Matter through the Higgs portal, Phys. Rept. 842 (2020) 1 [1903.03616].

[124] M. Duerr, P. F. Pérez and J. Smirnov, Scalar dark matter: direct vs. indirect detection, Journal of High Energy Physics 2016 (2016) . 